\title{
SMALL-MEDIUM ENTERPRISE EXPERIENCES OF FINANCIAL SERVICE IN URBAN AND RURAL SETTINGS: \\ A Phenomenological Study of a Changing Community Finance Landscape and the Sustainable Finance Narrative.
}

\author{
A Dissertation \\ Presented to the \\ Faculty of the \\ Graduate School at \\ the University of \\ Missouri-Columbia \\ In Partial Fulfillment of the \\ Requirements for the Degree \\ Doctor of Philosophy \\ by \\ CHRISTINE SANDERS \\ Dr. Mary Hendrickson, Dr. Hua Qin, \\ Dissertation Supervisors \\ JULY 2021
}


(c) Copyright by Christine Sanders 2021 All Rights Reserved 
The undersigned, appointed by the Dean of the Graduate School, have examined the dissertation entitled

SMALL-MEDIUM ENTERPRISE EXPERIENCES OF FINANCIAL SERVICE IN URBAN AND RURAL SETTINGS:

A Phenomenological Study of a Changing Community Finance Landscape and the Sustainable Finance Narrative.

presented by Christine Sanders, a candidate for the degree of doctor of philosophy, and hereby certify that, in their opinion, it is worthy of acceptance.

Dr. Mary Hendrickson

Dr. Hua Qin

Dr. David O'Brien

Dr. Harvey James 


\section{ACKNOWLEDGEMENTS}

I am sincerely grateful for the support I have received over the past few years. In particular, Dr. Mary Hendrickson and Dr. Hua Qin have been instrumental in any and all successes I have achieved along the way. Selfishly, I asked them to be my co-advisors, solely for the purpose of gleaning as much knowledge as possible per their respective areas of interest and expertise. They graciously obliged my request and collectively provided an experience that exceeded my expectations. I am eternally in their debt and also thrilled to have the honor to now address them each in a variety of ways - teacher, mentor, friend, colleague, to name a few. I am also extremely grateful to other members of my committee, Dr. David O'Brien and Dr. Harvey James, for their unwavering support, thought provoking and enriching feedback, and friendly encouragement. Beyond their invaluable contributions to this research study, David and Harvey graciously engaged me in projects that broadened both my professional and personal range, and for this I want to express my gratitude. It has been a privilege to have such a wonderful committee.

I am grateful to my husband, Jason, who I jokingly refer to as my unwilling supporter. In truth, my choice to pursue this journey shocked us both, but he has both respected and appreciated the commitment. In the end, no one has been more celebratory of my process and accomplishments. For this, I say thank you with all my heart. I also want to thank my parents, in-laws, siblings, nieces, and nephews. Luckily, I come from a family filled with love, endless support, and a taste for adventure. They cheered me on, even when the finish line seemed very 
far away. Lastly, I would like to thank all the study participants who made this research agenda possible. Without their generous time and support, the important questions would not have been asked or answered.

Certainly, I have learned many things throughout this process and am appreciative of the whole journey. However, perhaps the most meaningful aspect is that it re-awakened me to the limitless power of experiencing encouragement. So, lastly, a thank you to all - friends, family, and colleagues, old and new - who have ever encouraged me in any way. It means everything. 


\section{TABLE OF CONTENTS}

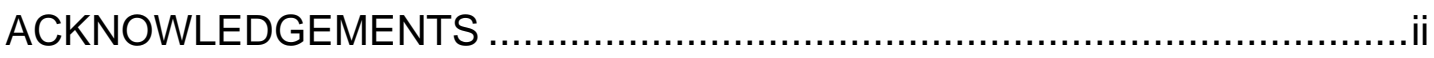

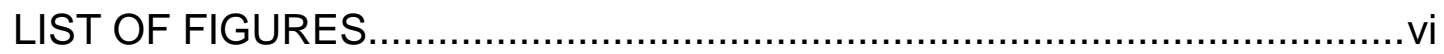

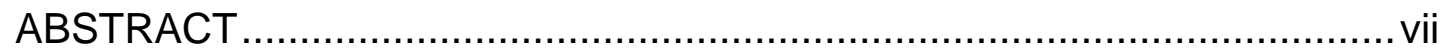

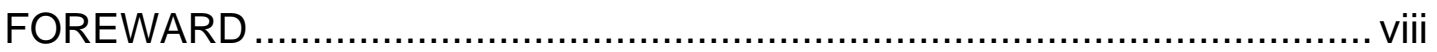

CHAPTER 1: INTRODUCTION, PROBLEM/OPPORTUNITY STATEMENT,

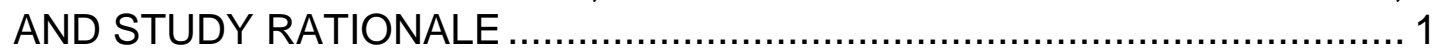

Statement of the Problem ........................................................... 4

Study/Rationale Purpose Statement …...................................... 9

CHAPTER 2: CONCEPTUAL FRAMEWORK ......................................... 13

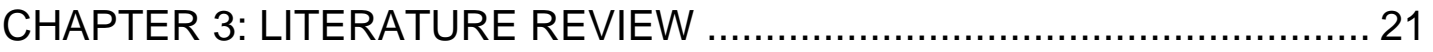

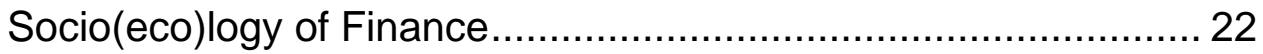

SME Finance, Relationship Lending, and Information ................. 26

Community Finance …....................................................... 30

Sustainable (and Social) Finance and CSR …............................ 33

Community Interactional Theory and Landscapes of Sustainable

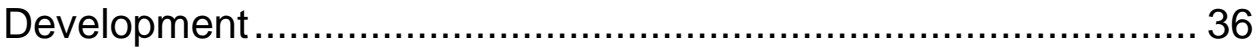

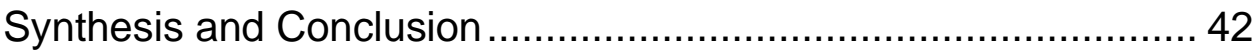

CHAPTER 4: METHODOLOGICAL APPROACH .................................. 47

Why a Phenomenological Inquiry? The Lived Experience Says A

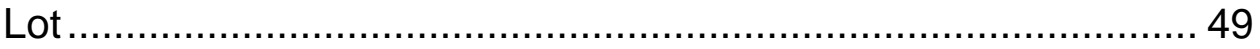

Hermeneutics and Phenomenology ......................................... 52

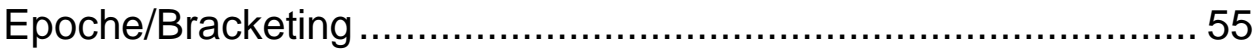

The Researchers' Role-As the Instrument and Navigating Epoche. 56

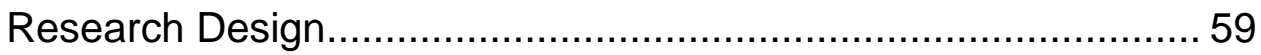


CHAPTER 5: FINDINGS

Setting and Population Representation........................................ 69

Findings Grouped by Research Question ................................. 76

CHAPTER 6: DISCUSSION AND IMPLICATIONS .................................. 108

Community Finance as a Process and Its Social Relationship Dynamics are Highly Valued. Sustainable Finance Is Missing (So Far) 112

The SME/Community Finance Experience May Be Knowledge "Light". Coupled with Technological Change, This May Be a (Future) Competitive Threat for Community Banks .

SME Finance Complexities (Will) Persist...Community Banks Need to Keep Up ........................................................................ 118

Community Finance is a Community Field. This Contributes Largely to Its Role in (Potential) Interactive Capacity Development Across the SDG Landscape 122

Yesterday's \& Tomorrow's Community Finance - Considering the Micro and the Macro for Resilience and Socio-ecologically Responsible Growth 124

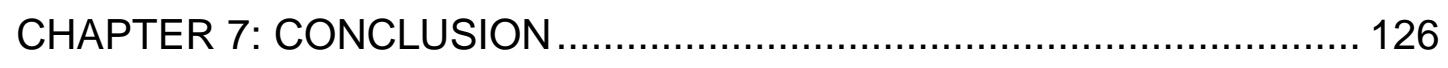

Small Matters for a Sustainable Future .................................... 127

Interactional Capacity and Context Matter ................................. 128

Community Finance Can be the "Grease" for Sustainable

Finance 131

Limitations 132

REFERENCES 135

VITA 155 


\section{LIST OF FIGURES}

Figure 1. Community Finance (for Sustainability) = Sustainable Finance (for

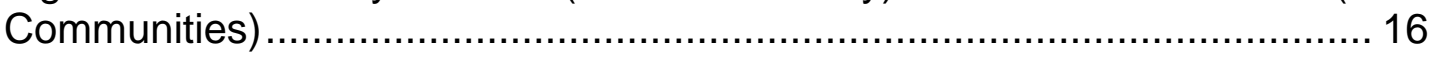

Figure 2. Phenomenological Analysis Approach........................................66 66 


\title{
SMALL-MEDIUM ENTERPRISE EXPERIENCES OF FINANCIAL SERVICE
} IN URBAN AND RURAL SETTINGS: A Phenomenological study of a changing community finance landscape and the sustainable finance narrative.

\section{Christine Sanders \\ Drs. Mary Hendrickson and Hua Qin, Dissertation Supervisors}

\begin{abstract}
Relationships between small-medium size businesses ('SME') and community banks are complex, meaningful, and often described as characteristically "opaque". This study offers a conceptual framework for exploring this "opaqueness" from the perspective of SMEs and community financiers engaged in the process of community finance. Insights into the experiential dynamics of SMEs and community financiers conavigating the financial service landscape offers an exploratory frame for investigations of interactive capacity, knowledge formation, and reflexive orientations. The implications of what transpires between SMEs and their community financiers can be far-reaching and of particular importance as the financial service landscape evolves to respond to calls for more 'sustainable finance' to support the goals of Agenda 2030, conveyed as the Sustainable Development Goals (SDGs).

The study findings suggest that multi-dimensional aspects of community finance processes, those both visible and invisible, are not well understood. Future research and practitioner ideation is suggested around the importance of placing community finance, as a facilitative process, within emerging 'sustainable finance' frameworks. Doing so offers an enhanced social-ecological lens for exploring the role, effects, and effectiveness of community finance in the $21^{\text {st }}$ century.
\end{abstract}




\section{Foreword}

In retrospect, I feel I was supposed to do this work, even without realizing it until well over halfway through the project. This research agenda is in many ways a culmination of life events, those both intentioned and those more accidental, all the same. The COVID19 pandemic required a slight research scope pivot, which in hindsight, pushed me toward a topic both of interest and of great importance to me. Upon reflection, I suspect my PhD journey, in this way, is not different than others who have embarked on this wonderful, maddening, and rewarding process. Nevertheless, I am grateful for the opportunity to explore the chosen topic(s) of community finance and sustainable finance, qualitatively. This study for me, and hopefully its readers, facilitates a very productive reflection on a segment of the broader financial service landscape that offers so much and in a variety of ways that are not always clear. What community finance has meant to those parties who engage with it in a host of contexts - past, present, and future - matters, arguably in both large and small ways.

Focusing the lens of inquiry on how small-medium size businesses experience community finance and sustainable finance, while in search of their relatedness (or not), is the result of one of those "accidental situations". A combined result of the COVID19 pandemic happening during my tenure as a $\mathrm{PhD}$ student/candidate and the livelihood challenges (and opportunities) it presented for my household. This is true for a couple reasons: 1) I am a coowner of a SME (my husband's appraisal firm), and 2) the COVID19 pandemic changed complexities and experiential dynamics, nearly overnight, for both small-medium businesses and their financial service partners. I know this 
because I personally navigated it. At the same time, my research interest was always centered on sustainability and sustainable development - both as narratives and practices. In a state of layered frustration (business and school navigations, personal/family health and safety), I had a very real moment where I asked myself a couple of questions: What populations are still open for business, or at least trying to be, including open to research engagements? What does community finance even really mean? The latter question arose due to my own, personal navigations with both a global bank and a smaller, community bank to work through our company's response to the COVID19 relief programs. At the same time, my academic interests were quite naturally converging with my lived experiences; community-level financial service interactions were critically contributing to the sustainability of my family's business and the broader societal landscape via very active responsiveness to the PPP program.

Sustainability narratives and initiatives, corporate social responsibility programming (both 'CSR' and 'ESG', organizationally speaking), and sustainable finance agendas, all affect and are affected by community-level dynamics. This research agenda highlights the importance of deeper inquiry into the latter, namely the effects of community-level dynamics and interactions on how sustainability linked goals are communicated, interpreted, and actioned (or not). At present, this is understudied and not well understood. Discussing this as a "missed opportunity" is the crux of the study, in particular since the findings lead in this direction. Hopefully, this initial scope of work offers beneficial insights into why that is the case, as well as invigorates the discourse on how to elevate 
community finance - the process and its stakeholders - into the global sustainable finance agenda. As you read this study, I urge you to consider why this is a topic of importance. What do community-linked financiers, or more specifically "community banks", do for not only small to medium businesses, but also society as a whole? What do they offer, culturally? What can/do they offer ecologically? Even with steady consolidation over the past few decades, community banks' resilience across the US landscape is notable. We need to understand why, and in particular, what happens if they cease to exist? In recent years, they have shown to be of great value in both "normal" business cycles, as they actively support entrepreneurialism and development in communities of need (e.g., rural or blighted), and those times that are considered "crises periods", such as the 2008 financial crisis and the COVID19 pandemic. Simply put, market-based evidence suggests community banks rise up in the face of adversity not only on their own behalf, but also for the benefit of their relationships and, in turn, their communities.

Personally, I am excited to continue on an ideation path centered on possibilities for both community financiers and SME leadership to become high value actors within sustainable finance frameworks. Engaged research from this vantage point will contribute to both domestic and international sustainable development agendas, and more broadly, the continued trajectory of shifting sustainability lenses from socioeconomic-first to more inclusive socioecologicalfirst principles. The exact nature of the relationship between small-medium businesses, community banks, and sustainable finance is perhaps not a 
completely answerable question. Nevertheless, just asking the question, repeatedly and as it relates to community finance's processual outcomes is what is critical. Arguably, this is what matters in terms of what comes next for $21^{\text {st }}$ century community finance and the millions of SMEs navigating our changing world. 


\section{Chapter 1: Introduction, Problem/Opportunity Statement, and Study Rationale}

Small-medium enterprises (SMEs) and financiers, alike, often have community-level identities and engagement practices that are of primal interest to this study. The sustained interactions between small businesses and their financiers are observable as essential components of the relationships that form between these actor groups, often traversing both personal and professional scopes of interest. Depending on both scale and strategy, SMEs and communitylevel financiers (e.g., community or neighborhood banks) can sometimes be powerful social actors (especially in rural settings), and interestingly, have largely sustained one another over the past several decades. Their relationships are dynamic, and characteristically involve some level of trust, two-way information sharing, and shared objectives of value creation at both the enterprise and community level. At present, this landscape is changing due to a variety of factors - technological change, new hazards (e.g., climate change, pandemic), regulatory design, and persistent market pressure (e.g., consolidation, de novo banks). The emergent sustainable finance narrative and its related frameworks are another materialization community banks and SMEs now are called upon to jointly navigate. The essence of if, when, and how they may do so is the crux of this research project and the rationale for exploring SME finance (financial service activity between SMEs and community banks).

Financial theory and practice continue to evolve in necessary ways to address its contemporary meaningfulness. No question, finance, when 
considered as either a verb or socio-economic actor, makes things happen. Often this is in transaction-oriented frames, but when looked at more inclusively and with context specificity, it becomes clear its reach is both deep and wide. As an example, we must consider its central placement in proposed operationalization of vast project activity in the name of the Sustainable Development Goals (SDG). Finance's influence level is observable as similar across enterprise profiles, industrial sectors, and communities, however this an oversimplification of both what it does and what it can offer. The existing study extends the discourse on what the industry's role is in the $21^{\text {st }}$ century by placing community finance, as a processual experience, as the subject matter of priority interest for exploring sustainable finance meanings and activeness in firms and communities. In addition, a purposeful selection of small-medium businesses as the study sample population offers a platform for contextual discovery that can provide insights on perceptions and behaviors of enterprise leaders that, on a collective scale, are extremely influential and understudied. Furthermore, SMEs are not exempt from the potentially seismic shifts occurring within $21^{\text {st }}$ century finance as part of the industry's broad responsiveness to changing environments and landscapes, globally. Sustainable development agendas and evolving socialecological financial service models across the human-environment system will significantly affect both community banks and SMEs, as well as need their support.

A qualitative inquiry was designed and deployed to explore the smallmedium enterprise (SME) experience of community finance in a changing 
financial service landscape. The objective is to invigorate both the thought work and dialogue on the complexities of SME finance, community finance, and why it may matter in outsize ways. This study highlights the experiential characteristics of community finance (the process) and any evidentiary relationship to the emerging sustainable finance narrative and frameworks by exploring SME and community banker shared experiences of financial service. A conceptual framework that posits community finance as most suitably embedded within the sustainable finance narrative was utilized in an attempt to theorize both intentional and unintentional consequences of SME finance interactions at the individual, enterprise/organization, and community level. The research question focused on a few critical aspects of the SME finance experience and $\mathrm{SME} /$ banker relationships. First, what is community finance, from the perspective of SME leaders? Second, what is sustainable finance, from the perspective of SME leaders? Finally, how are they related, experientially? The research explored any observable links between community finance and sustainable finance in terms of both the dynamics of their lived experiences and indicated exposures to the sustainable finance narrative and/or projects.

The study findings suggest a healthy community finance ecosystem remains critical to the growth and resilience of small-medium enterprises and vice versa. SMEs highly value their community bank relationships, and community bankers want to do business with SMEs in their respective communities. Characteristics of nimbleness, active information sharing, loyalty, caring, and trust were observed across most relationships. Nevertheless, at the 
time of the study, community finance and sustainable finance were generally perceived as separate topics for both SME leaders and community bankers. In particular, this seemed to be consistent with no awareness of what sustainable finance is supposed to represent. This study makes a considerable contribution to the conceptual framework for continued scholarship and policy development to better connect SME finance and community finance, as a process, to the sustainable finance agenda. Positive implications for enhanced social-ecological leadership and inclusive project design for sustainable financial service systems can be the result.

\section{Statement of the Problem}

\section{Background}

Why small-medium size business? Small-medium enterprises (SMEs) are critical to the US economy's well-being, and collectively, are no small social actor group. There are over 30 million SME businesses in the US, and they employ approximately $47 \%$ of the American workforce (advocacy.sba.gov, 2020). Furthermore, this profile of companies, which includes operations employing anywhere from one to 500 persons, is often credited with innovation and job growth with a greater frequency than large-scale enterprises (McAdam \& Keogh, 2014). On both national and state levels, SME leadership offers an important and arguably powerful representation of America's economic and social identities. SME owners/leaders are of diverse racial and gendered profiles, they are entrepreneurial, and they are often actively involved with their communities. SMEs may also be deeply and broadly connected to multiple networks (e.g., 
global value chains, religious organizations) across much larger socio-cultural landscapes.

On local scales, SMEs can be significant employers with their leadership highly involved in not only the business, but also the governance of regions. Categorically, these firms are comprised of a broadscale set of characteristics, and, regardless of size, they require active service relationships with their financial service providers (advocacy.sba.gov, 2020). Thus, SME finance is not a niche business for financiers of any scale. Financial institutions of varied size, profile, and location strategically work to meet SMEs' operational requirements, potentially throughout the entirety of the enterprise's lifecycle. As a result, financiers involved with SMEs often have some level of influence on SMEs' socio-economic outcomes, strategic maneuverability, salience, and overall resilience, suggesting the relationship dynamics between SMEs and their financiers extends beyond the realms of economic principles and firm-level financial performance.

Why community banks? Smaller scale financiers, or community banks, are arguably quite affected by the clients with whom they do business. This can depend upon a variety of factors, such as bank ownership structure, service engagement terms, etc. Transactions between SMEs and their financiers can be nuanced and complex, built upon a bedrock of information exchange, trust (or distrust), and market factors. Better understanding the aforementioned in context offers benefits for shifting the SME finance discourse from an economic orientation to a social-ecological one. With its fluidity and semi-flexible design, 
community finance is most easily described as an opaque process of information exchange and decisioning that is facilitated by a variety of mechanisms. In addition, as evidenced by both the 2007-2008 financial crisis and, more recently, the business challenges that arose due to the COVID19 pandemic, community finance may be uniquely positioned for leadership and participatory action pertaining to both business and community-level sustainability and resilience.

Why sustainable finance? Over the past decade, sustainable finance has aggressively entered both the lexicon and practice of finance. To date, one of its shortcomings is the selectivity by which sustainable finance products are developed and deployed. Yet, narratives on sustainable finance are encouraging as they urge more inclusive operationalization for broadscale, positive social and environmental outcomes. Exploring aspects of sustainable finance exposure on behalf of SMEs is critical to unpack how it is/is not becoming relevant to their current financial service experiences with community banks or other financiers.

Why reference the Sustainable Development Goals (SDG), and in particular SDG17? Partnership is a commonly used term in sustainable finance narrative and development projects. The SDGs are often invoked by large-scale, private sector actors in evaluation, marketing, and stakeholder communications. Arguably, this is a positive outcome for aspirational norms around truly sustainable finance. As described by Mendez \& Houghton (2020), "SDG17 specifically envisions partnership between the public and private sector for collaborative finance" (Mendez \& Houghton, 2020). The application of SDG17 for this research agenda adopts this viewpoint yet focuses on the merit for 
community level design as opposed to partnership-only orientations for project deployment and evaluation processes. SDG17 and operational partnerships are vitally important for progress across the entire SDG framework. Thus, the argument is not to discard partnerships, but rather elevate the visibility of the community level context of their engagements.

\section{The Problem (and the Opportunity)}

Finance's evolving scope of interest has very real implications for SMEs, community banks, and the communities within which they operate. Greater insight into SMEs and community bank leader salience and levels of activeness in response to the shifting financial service landscape may offer new ideas for sustainable development. What SMEs and their financiers (bankers) "think" and "do" affects both enterprises, and community-level responsiveness to change and can have local market implications, result in new network formations, generate knowledge gains/losses, and ultimately shape the social, ecological, and cultural landscape. SME finance, or SMEs and community banks engaging in the process of finance and its normative aspects often include relationshipbased exchange of information and values, time sensitive credit facilitation, and creative collateralization processes. It is in this context that SME finance is representational of community finance's socio-cultural and ecological reach and the catalyst for exploring its impacts. In support of this, it is important to clarify a few central points. First, SMEs and their respective financiers are physically located everywhere; in large/small municipalities, urban and rural settings, and in places of urban/wildland interfaces. Second, SMEs conduct business with 
financiers of varied size and scale that purport to be community financiers. Finally, technological advancements have enabled distant community financiers to engage within historically more local system. Thus, community finance in the $21^{\text {st }}$ century can arguably be described as possible everywhere. As further evidence, financiers that are involved with SMEs, especially those found within specific neighborhoods and/or more rural settings, are often explicitly identified as community-level actors. This is observable in terms of enterprise names (“Community Bank of X"), marketing communications ("Bank X, your friendly community bank"), and at times, a combination of name and positioning within a town, neighborhood, or community of shared subject matter or industrial interests ("Bank of X" on Main Street, USA, or "Bank of X" focused on "Industry Y"). This suggests there is a sizeable presence of community in finance, an inference that offers both problems of departure and opportunities of great alignment, depending upon what is actually occurring in practice.

Second, multi-disciplinary scholarship describes finance as the "the grease" for economic and societal progress. Scholten's (2006) approach for its framing as providing the "transmission mechanisms" for sustainable development offers a useful visual aid for considering its attributes and strengths, both in theory and practice (Scholten, 2006). These mechanisms are not well understood. Furthermore, relationships between perceptions and behavior are dynamic and may consist of bi-directional feedback (Qin et. al, 2021), thus consistent, qualitative inquiry into situational change events can offer useful insights that may be missed or undervalued in quantitative-only analyses of 
finance's characteristics and contributions. At present, subsets of the finance literature, such as relationship banking and sustainable finance studies, tend to address practices of assessment, project/product characteristic, and/or precursory or outcome variables (e.g., relationship or what type of information gets exchanged) in explorations of finance's socio-economic and cultural contributions, as opposed to engaging with the transmission mechanisms themselves. Importantly, how the mechanisms of financial service interactions and eventual value exchange (or not) not only arise but also change over time at individual, enterprise, and community levels is a complex and dynamic process. Better understanding the mechanisms may provide insights into social-ecological developments that may be beneficial to financial service processes in changing environments and landscapes. As both community banks and SMEs can be found as influential, embedded social actors at the community level, investigating their shared processes by which all types of knowledge and ideas may be exchanged seems quite appropriate. Meaningful insight into community finance's (and sustainable finance's) financial and non-financial interactive capacities for supporting sustainable development, both locally and globally, is the desired result of the endeavor.

\section{Study Rationale/Purpose Statement}

Studying community finance, fundamentally as a process that is continuously co-experienced by SMEs and community bankers, offers a new perspective for exploring SME finance, community banking, and relationship banking, and their relation to (or not) sustainable finance. In particular, the effects 
of finance's relational dynamics and interactions, whether intentional, unintentional, formal or informal, regarding both individual level and community level attitudinal, knowledge, and habit formations, seems understudied. In addition, community finance's role and/or placement within emerging sustainable finance frameworks is not well understood, and arguably, not being properly considered. Given the widely applied descriptive labels of "community banking" and the increasing presence of "sustainable finance" as a more recent, aspirational attribute of what finance does, is, and offers, it is critical to understand more about how these topics can/should/do converge. The purpose of this study is to explore the complex and contextual differences that are observable on the surface of financier/client relationship dynamics, to consider what is visible (e.g., transaction scope/information, actor scale, geopolitical influences), and yet also extend inquiry to that which may be described as the "invisible". The latter elements which are constructs such as essential knowledge, idea generation/management, and attitudinal formations that may result from the experience of the financial relationship/partnership are of exceptional importance for deeper investigation into the holistic essence of the relational finance experience. Furthermore, a key objective is to identify what may be invisible on the surface; and hence, how it may have the potential to become visible via what is decided, chosen, actioned, or prioritized by SMEs. Allowing for an arguably fictitious separateness to exist between community finance and sustainable finance presents problems and missed opportunities. As an example, there is a sizable activity and active celebration 
regarding the growth of impact investing and social investment products (Hebb, 2013). Yet, as cautioned by E. Jackson (2013), we should be careful not to oversimplify or be too selective in variables of measurement that can be misleading or exhibit partiality if context is not well understood (Jackson, 2013). For decades, modern finance theory (MFT) has been critiqued for not robustly addressing moral and ethical dimensions of its presence (Singer, 2009). Financial products and or solutions that are too narrow or omitting selective impact characteristics are problematic in both visible (greenwashing) and most likely invisible (knowledge deterioration) ways. Arguably, the placement of community and community related processes, such as the interactive nature of community finance and its transmission mechanisms, could contribute to the addressment of these types of challenges. Specifically, sustainable finance champions and industry leadership should address this in a priority capacity. By seeking out dimensional aspects of multi-scale actor relations and socio-cultural nuances that may exist in pockets of a landscape, to identify and redirect "small things" before they become "big things" that can have vastly negative implications. This study works to establish an initial framework to do so through the elevation of the community interactional lens.

Finally, this study incorporates the pandemic related experiences of SMEs and community banks. The COVID19 pandemic prompted a variety of responses at individual, local, regional, national, and global scales. From an industrial lens, no sector escaped at least some degree of impact, whether in operations, client relationships, workforce, or other stakeholder interactions. Within the finance 
sector, the pandemic unleashed a flurry of activity that arguably has not been seen since the 2008 financial crisis or before. Policymakers, lenders, and clients were faced with challenges ranging from currency stabilization to market liquidity to shifting consumer dynamics. Due to its linkage to every other industry, finance was a central actor (both gatekeeper and co-architect of mechanisms) for government sponsored risk mitigation solutions. Financiers, collectively, were effectively the delivery system for fast-engineered economic safeguards in the midst of an economic storm that necessitated a lot of trial and error. One such program, the Payroll Protection Plan (PPP), was facilitated by banks and other types of lending organizations. PPP has been a lifeline to SMEs throughout the COVID19 pandemic, thus it offered a meaningful reference indicator for examining the dynamics of relationships between SMEs and community banks when jointly navigating a changing, and to some extent crisis oriented, landscape.

The study's purpose is to discover the essence of community finance dynamics between SMEs and community financiers by looking deeply into their relationships in both routine and crises (COVID19 pandemic) navigational frames. In addition, the question of what this does or does not have to do with emerging sustainable finance narratives and frameworks is an underlying and purposeful theme. 


\section{CHAPTER 2: Conceptual Framework}

Drawing from the community as social structure and interactional conception of community literature that grew in prominence during the mid- $20^{\text {th }}$ century and continues its evolution today (Kaufman, 1959; Luloff, 2019; Sanders, 1966; Wilkinson, 1999; Dickinson \& Hawley, 1950), a framework for exploring community finance as an interactional experience and its potential for embeddedness within sustainable finance offers valuable insights to bank leadership, policy makers, development planners, and small business owners. This seems especially important, as community is often present in marketing, performance, and sustainability narratives of the finance sector.

The dimensions of how community banks engage with SMEs and community finance's possible/probable relationship with sustainable finance in both narrative and practice are quite relevant to businesses in both normal and crises periods, such as a during the COVID19 pandemic. Sustainable finance frameworks for progress, project implementations, and product breadth are multiplying, seemingly daily, and may exert real change pressure on community banks in the years ahead. Due to its priority tenets of partnership, transparency, and to some extent, entrepreneurialism, sustainable finance inevitably overlaps in its modeling, as compared to community finance. Thus, this only adds to the complexity of such affective change having implications for community banks and their stakeholders, with SMEs being one of the largest collective groups that works closely with this financier type. The study's framework was designed with a first-things first approach in mind to encourage thoughtful consideration on this 
factor by focusing on the interactive experiences of SME finance. Questions about how SMEs and community banks experience their co-relationship lays the groundwork for a conceptual roadmap on what is occurring today, what is potentially being missed, and what becomes possible tomorrow for more inclusive community finance that centers on social-ecological prioritizations and symbiotic human-environment systems.

The conceptual framework for this study places community finance as central to sustainable finance on an exploratory basis. Community interactional and pragmatism theories largely inform the conceptual framework, with notable appreciation for recent works by Mainzer and Luloff (2017) and Migliorelli (2021) that direct toward a "community landscape theory of pro-environmental behavior" (Mainzer and Luloff, 2017) and "finance for sustainability" (Migliorelli, 2021), respectively. In addition, SDG17-Partnerships for the Goals is called upon as a reference mechanism for positing the necessary and bonded relatedness of community-level and partnership dynamics. SDG17 explicitly states its objective is to "strengthen the means of implementation and revitalize the global partnership for sustainable development" and places finance as a central, critical resource (The Sustainable Development Goals Report 2019, 2019) for success. SMEs represent a large community finance stakeholder group and are found to be active in partnerships, both formally and informally, making them the priority population of interest for this study's framework that offers logical and practical merits for embedding community finance within sustainable finance. 
The conceptual framework relies on a few key assumptions. First, it indirectly asks the question, what is a community? There is no absolute definition or fixed form of a community. This research agenda prioritizes its conception as a social structure consisting of socially organized interactions, both with and without intentionality, and no fixed spatiality or temporal constraints. Furthermore, communities are multi-dimensional structures that involve both interactions and shared experiences. In addition, the conceptual framework affirms Lash's description of communities' "reflexiveness" and Hawley's rationale that community-level distinctiveness originates from "daily life rhythms" (Delanty, 2003; Wilkinson, 1999).

Second, community finance constitutes a community field. According to Wilkinson (1999), a community field,

...has actors, associations (both organized and unorganized), and activities directed toward certain interests, but in the community field, unlike the others, the interests are generalized and intrinsic; they are not specialized or instrumental. The community field cuts across organized groups and across other interaction fields in a local population. It abstracts and combines the locality-relevant aspects of the special interest fields and integrates the other fields into a generalized whole. (Wilkinson, 1999) Notably, with the advent of major technological advancement over the past couple of decades, this is modifiable and extendable to include virtual communities. 
Third, financiers and SME leaders are embedded in communities as community-level social actors.

Fourth, sustainable finance must result in beneficial outcomes inclusive of human well-being, profit achievement and/or maintenance (or equivalent market aligned metrics), and biophysical vitality. Valued and measurable factors for each include both visible and invisible outcomes (Goglio \& Catturani, 2019).

Fifth, interactive capacity is perceivable and socially constructed; therefore, it can be meaningfully real as co-experience, co-habit, co-knowledge, co-belief, and co-identity formations at the individual level, enterprise level, and community level.

Sixth, sustainable finance is not possible without community-level engagements. The SDG17 partnership narrative is too narrow and risks generating silo effects and alienation problems.

Finally, community finance for sustainability equals sustainable finance for communities.

Figure 1. Community Finance (for Sustainability) = Sustainable Finance (for Communities)

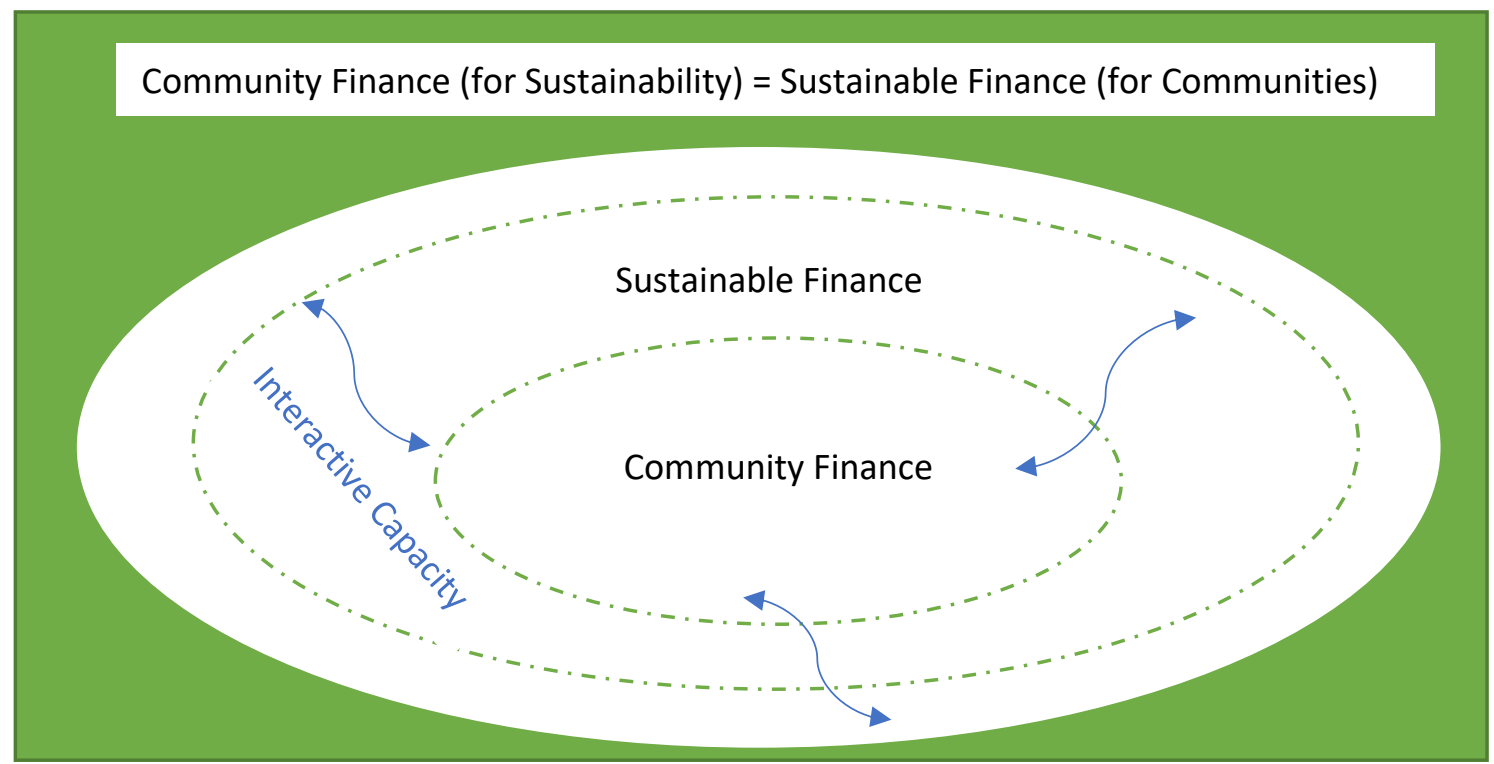




\section{Epistemology}

Central to my research is the assumption that interactive capacity is present and active across SME and financier relationships and that it facilitates knowledge creation in a continuous cycle. Interactions between such actors are observable as active, repetitive, information rich, social in nature, purposefully motivated, and typically place-based or within an identifiably structured community identity. Furthermore, this study is grounded in a belief that the concepts of sustainable development, community development, and corporate social responsibility (alternatively ESG) may benefit from inquiries with a lens that focuses on the interactions as the mechanism(s) between actors for the purpose of securing or providing financial capital for livelihood pursuits. Researching the community finance and sustainable finance interactional landscape as experienced by small-medium business owners and community banks offers a lens for probing what is occurring, and being experienced, both within the relationships and the communities. Classical pragmatism posits that meaning comes from experience, and this research framework largely accepts this ontological perspective. As the sustainability discourse is arguably one of the most important topics of the $21^{\text {st }}$ century, it is the viewpoint that an opportunity exists for more and faster progress if contextual insights into how the normative processes of small-medium business financing contribute to perceptions, knowledge and attitudinal formations, and behaviors related to sustainability are incorporated into sustainable finance's systemic design. 
Earlier work by Mainzer \& Luloff (2017) conveys landscape as a socially constructed place that comes to exist by way of dynamic perceptions that arise from individuals interacting in social environments, thus highlighting landscape as reflective of normative formations of the involved community and its emergent culture - past, present, and in the future. This offers a beneficial viewpoint for the current study's utilization of landscape as the perspective from which to consider its range of possible impact. Individuals involved with community finance are doing so in socially constructed places, regardless of the exact interactive format, place, or level of pre-existent capacity. In drawing from Wilkinson, there are at least three distinctively important points to be made from this. First, landscape and community are inextricably linked and in states of constant creation and recreation. Second, there is an unbound time horizon including both past and future outcomes. Third, there exists an ever-presence of social interactions in varied forms, both conscious and unconscious, in community interactional processes (Mainzer \& Luloff, 2017; Wilkinson, 1999). The landscape of financial service interactions provides a rich data source for experiential inquiry as financing is something most businesses do as part of normal operations. In addition, the COVID19 pandemic has presented a unique opportunity to also examine SME and financier interactions during a period of heightened hazard and risk response as part of business and livelihood resilience. Exploring how financial entities are meeting the needs and expectations of business owners, or failing to do so, offers further comprehension on the interactive dimensions and dynamics of community finance landscapes and how they are related to the 
social-ecological imperatives linked to sustainable finance. Individual recall of experiences in comparative frames of normal and crisis orientations provides a supplemental conceptual mapping for the relationship dynamics and response decisioning.

Financial supportiveness is an acknowledged, often necessary component of viable sustainable development. If within landscapes of financial experience, an opportunity for increased knowledge and information capacity development across communities of all shapes and types can be tapped as part of the broader sustainability agenda, a tremendous amount of knowledge may be exchanged. The meaningfulness of sustainability, community development, sustainable development, and social responsibility is an increasingly important facet of the broader sustainability narrative. Unpacking actor accounts of one aspect of sizable influence upon the daily rhythms of collective life seems to be a very appropriate vantage point from which to explore how individuals and communities are developing salience (or not) for sustainable finance via community finance activities (Balfour \& Alter, 2016; Dickinson \& Hawley, 1950; Hemingway, 2019; Klassen \& Vereecke, 2012; Mainzer \& Luloff, 2017; Wilkinson, 1984).

This conceptual roadmap attempts to contribute to the collective knowledge system concerning stakeholder needs and receptivity for "more than transactional finance" from their community banks in the $21^{\text {st }}$ century. With massive social-ecological and economic shifts forecasted, there is an opportunity to explore community finance as a priority mechanism by which real and 
measurable progress is possible in every place, or space, finance is critical for successful implementations related to the SDG framework. 


\section{CHAPTER 3: Literature Review}

A systematic review of literature in the areas of SME finance, relationship banking, community finance, sustainability, CSR, and sustainable finance largely informs this study. Various theoretical scholarships also supported the development of the research concept. The supplemental literature included articles on sociological theories of finance and markets, finance ethics, and community based natural resource management, and social investment. Finally, the conceptual framework places the process of community finance, as well as its social actors, as embedded within the sustainable finance landscape to theorize their role and potential for positive developments associated with the latter. It is posited community finance processes yield transmission mechanisms that affect perception, attitude, habit, and meaning formation via both intentional and unintentional interactive processes between community financiers and their SME relationships. Thus, seminal works on community interactional, social capital, organizational theory and design, landscape, and classical pragmatism were important for the development of the conceptual framework.

The articles and books were primarily obtained via Scopus, Google Scholar, and the University of Missouri libraries. The initial review focused on establishing a foundational understanding of prior works on the topics of interest. Subsequent searches were more concentrated within the subject matter verticals that supported the research questions and conceptual design. In line with the selected methodological approach, which is hermeneutical phenomenology, a

post-project search of the literature was also completed. A prioritized collection of 
literature that both informs and supports the study design, its key conceptualizations, and the interpretive discussion is presented below.

\section{Socio(eco)logy of Finance}

Financial theory literatures confirm what is largely observable about finance as an industry. Its structure represents a global network of multi-tier interconnectedness, established governance systems, formal and informal actions, indications of high influence by both banker relationships and social networks, increasing trends of digitization and remoteness (particularly in rural settings), and broadscale embeddedness in society (Berger \& Udell, 2006;

Carruthers \& Kim, 2011; Elyasiani \& Goldberg, 2004). Financial organizations are quite diverse in structure, including firms such as banks, credit unions, and assetspecific funding vehicles (e.g., large equipment funders and specialized venture capital). They are deeply involved with social, economic, and geo-political forces on multi-level scales due to their participation in institutional information curation and dissemination, economic development prioritizations, technological innovations, market structures, and governmental policymaking across the interconnected, global environment (Berger \& Udell, 2001; Carruthers \& Kim, 2011; Li \& Foo, 2015). Even more diverse are the client profiles of financial institutions, which range from sole proprietors to global enterprises of massive size and global reach. Yet, much of the financial literature to-date avoids direct assessment of the diverse complexities that emerge within such an expansive group. Procedural governance principles are regulated and inherent in financial institutions, affecting similar clients in similar ways. This is understandable when 
considering the problems that can arise if/when some version of a shared regulatory framework is not adopted to protect the interests of both the industry and its constituents. At a macro-scale, and quite importantly, the rich library on investigative themes of finance's role in socio-economic advancement has greatly contributed to the collective understanding of its criticality. Examples include correlative performance indicators, credit accessibility for specific populations, environmental risk and financing product innovations such as impact bonds, and geo-political stabilization effects (Rizzello \& Kabli, 2020; Secinaro et al., 2021; La Torre et al., 2019; Wu \& Shen, 2013). Attributes of the finance industry's deep entrenchment and powerfulness in market design is well documented.

Yet, there remains an active and incomplete discourse on how finance's contextual attributes are critical for understanding layered responsiveness, biophysical connections, relationships, narratives, and attitudinal responses to the global sustainability agenda. Modes of information exchange, trust formations, and service interaction models, including those transitioning to low or no touch systems (e.g., online only) are characteristic of context variability in financial engagements (Mukherjee \& Hath, 2003). Thus, findings in the current literature contain mixed results and suggest a need for continued research on relationship banking and its role in modern finance experiences. Beyond individualized case-level attributes, the rapid technological changes are also affecting community-level financial interaction processes, regardless of 
community size or profile, and need to be contextually explored (Mendez \& Houghton, 2020; Pink, 2011; Willson, 2006).

Largely, the finance industry's role, both in historical and current contexts, has been to create and/or facilitate the creation of and preservation of value on behalf of its stakeholders. A variety of mechanisms are in place to balance efficiencies and value orientations between society and the natural environment (Rezende de Carvalho Ferreira et al., 2016). Inherently, this structuration is not problematic in the context of what finance does and offers, but rather necessitates a general care for how value, efficiency equations, and performance targets are approached, developed, measured for, and duplicated. Nevertheless, ongoing risks of moral hazard and unethical orientations toward convenient narratives, and sometimes even willful misconduct, in approaches to what is valued (e.g., profit and/or efficiency calculations) can persist and yield negative effects. Relevant to this study, examples in the literature of negative outcomes resulting in misaligned, or un-sustainable financial service include cases of "dirty banking", greenwashing, unethical practices, and exclusionary products and principles which are in opposition to fair and inclusive outcomes critical for sustainable development across the human-environment system. (Archer, 2012; Urban \& Wójcik, 2019). Furthermore, if contradictory agendas arise, regardless of intention, it could be the case that financial actors are to be found embedded in both the problem(s) and solution(s) due to their influence and reach and alignment with other giant actors, such as the United Nations. Finance can punish or, more interestingly, reward with its capital flows, as evidenced by 
findings of correlative lower capital constraints and higher CSR performance that can include both social and environmental dimensions of CSR (Cheng et al., 2014; Utting \& Zammit, 2009). This makes it an outsize influencer in the overarching structural design of global sustainability agendas. Arguably, this very point is primarily why SDG17 exists and why continuously engaging on the question of finance's role as a social-ecological actor is important.

Galaz et al. (2018) offer a beneficial sight line for considering finance's role in the $21^{\text {st }}$ century, proposing an ecological methodology "to identify the ways in which financial actors and flows of capital are linked to biomes associated with key tipping elements in Earth's climate system"(Galaz et al., 2018). Conceptually, this approach offers a facilitative viewpoint from which to consider finance's role beyond transaction-, innovation-, or product-first scopes (e.g., green or social bonds, impact investing products) and shifts the focus to the human-environment system, highlighting adaptation pathways for values modifications toward improved social-ecological outcomes, and a more participatory engagement framework across the complex system. Arguably, this may support finance's modern trajectory as a key, lead actor in the direction of meaningful sustainable development, nicely described by Bolis et. al (2014) as development that is tri-dimensional: "satisfaction of human needs (including social and economic aspects), natural resources (making explicit Earth's limitations, and decision-making perspective" (Bolis et al., 2014; Butler et. al, 2016). Arguably, it is Bolis's third dimension that is the primary area of interest for this research agenda, particularly since it calls attention to dynamics that should 
not be ignored in relation to advancing sustainable development. Financial service processes, especially those related to credit decisioning, for SMEs and bankers engaging as community-level actors, at present, are very human-centric.

\section{SME Finance, Relationship Lending, and Information}

SMEs were selected as the population of interest for the study for a few critical reasons, primarily due to their independence in decision-making, high level of involvement with community banks, and collective group size. SBA data indicates there are 30.7 million small and medium-sized businesses in the United States, employing approximately $47 \%$ of the American workforce. Furthermore, this employer profile is credited with much of the innovation and job growth in recent years (advocacy.sba.gov, 2020). The compositional attributes of SME finance, as demonstrated by the financiers and SMEs engaged in financial service processes are diverse, complex, and multi-scalar. It is important to note, all types of banks participate in SME finance, from local to global, de novo banks, fintechs, and more specialized firms such as equipment finance and financial risk and advisory firms (de la Torre et. al, 2010). Banks, credit unions, cooperatives, and other financial service enterprises provide generally similar services to the SME community (e.g., credit, debt, depository services, etc.). These service providers include large, regional, small or local, asset specialized, global, brick and mortar, online-only, privately-owned, and/or publicly traded enterprises (Costa-Climent \& Martínez-Climent, 2018; Forcadell et al., 2019; Jeucken, 2010; Nosratabadi et al., 2020; Raslavich et al., 2020; Yip \& Bocken, 2018). 
Analytical frameworks concerning SME finance have historically focused on the availability of external finance for SMEs as categorized into 2 types transaction lending (hard information) and relationship lending (soft information) (Berger \& Udell, 2006; Santikian, 2014). The evolving academic library on financial intermediation (banking services) and relationship lending supports the need for continued study on how customer-to-lender relationships should be considered as critical factors in causal chains across financial framework developments and policy, particularly to challenge information asymmetry, oversimplification of modeling assumptions, and more inclusive strategies (Berger \& Udell, 2006). The vast literature, to date, acknowledges the primacy of soft information gathering as the key characteristic element of bank service offerings (Boot, 2000; de la Torre et al., 2010; Elyasiani \& Goldberg, 2004; Hale, 2012; Hussain et al., 2021; Lehmann \& Neuberger, 2001; Santikian, 2014; Srinivasan, 2014; Uchida et al., 2012). Several studies point to soft information as having positive effects on customer borrowing capacity, rate favorability, collateral requirements, product reach, and program access (Berger \& Udell, 2001; de la Torre et al., 2010; Elyasiani \& Goldberg, 2004). Historically, the power of the lending relationship, and relatedly its designation as the soft information access point has occurred regardless of financial institutional size or structure (Uchida et al., 2012).

Loan officers play a critical role in soft information production. This contributes to banks' comparative advantages, yet also necessitates a critical lens for avoiding exploitation (Boot, 2000; Hussain et al., 2020; Uchida et al, 
2012). SME finance activities are firmly placed within this unpredictable landscape as SME leaders work closely with primary (and supplemental) points of contact in financial entities to execute transactions in accordance with perceived needs. The literature labels such engagements "relationship lending" or "relationship banking", pointing to factors of trust, temporality, and both hard and soft information exchanges as key components for productive outcomes desired by the involved parties (Bright \& Manfredo, 1997; Kautonen et al., 2020; Martínez \& Rodríguez del Bosque, 2013; Mukherjee \& Nath, 2003; Rauterkus \& Munchus, 2014). Interestingly, crises have historically yielded negative impacts on new relationship formations; however, community banks do not follow this trend (Hale, 2012). Findings by Santikian (2014) and Donker et al. (2020) may offer insights on this countertrend, whereas respectively, their studies point to the vastness of soft information's influence, specifically service-level cross-selling, relationship expansions, and post-crises and duress lending (Donker et al., 2020; Santikian, 2014). Mixed results on the effectiveness of banker-SME relationship contributions at the individual, firm, and community level are present across the literature.

SMEs have been documented as creative, innovative, knowledgeable, entrepreneurial, risk-taking, and collaborative in adaptive and nonlinear formats (McAdam \& Keogh, 2004; Turner \& Endres, 2017). A recent working paper by Fairlie (2020) highlights the experiential variations of SMEs as they respond to the COVID-19 pandemic, offering a current landscape for discussing SME heterogeneity in the context of varied experiences of financier and financial 
product resource accessibility (Fairlie, 2020). Similarly, reviews of SME interactions with financiers during periods of financial crises over the past few decades highlights unevenness in information and network developments, with the latter exhibiting signs of weakness in both reach and related product (credit) access that may greatly contribute to SME leader/owner distresses (Donker et al., 2020; Hale, 2012; Santikian, 2014). Less prevalent in the literature is a clear discourse on finance's ethical dimensionality and its interaction effects on client industries and customers as it responds to evolving characteristics of resource need for clients navigating a changing socio-economic landscape and new emerging risk frames. Qualitative insights on the role of what is needed by both the financial institutions and their clients, which extends to small-medium business firms, are described as much needed additions to future research (Beck, 2013; Berger \& Udell, 2006; Carruthers \& Kim, 2011; de la Torre et al., 2010).

Additionally, the discourse on the impacts of technological advancements and bank consolidation on the characteristics of lender-client relationships exhibits mixed findings with regards to length of relationship time, physical embeddedness within a community, and degrees of trust each notably regarded in the literature as important for a SME lenders' comparative advantage (Elyasiani \& Goldberg, 2004; O’Hara, 2017). Concerns about negative factors of SME finance factors have emerged in the relationship lending literature, with examples such as soft information working against the client, exploitive practices, 
and limited capacity on behalf of SME lender understanding and entrepreneurial responsiveness (Hussain et al., 2021; Marquis \& Lounsbury, 2007).

The SME finance scholarship seeks further contributions of SME finance studies that explore the importance of networks, marketing navigations, navigational effectiveness in challenging times, problematic one-size-fits all transactional design, and addressment of enterprise-specific opaqueness (Berger \& Udell, 2006; de la Torre et al., 2010; Turner \& Endres, 2017).

\section{Community Finance}

The community finance literature is not fully separatable from SME finance as there is significant overlap between them. As an example, relationship banking is a central theme across both SME finance and community finance literatures; whereas the information exchanged as part of financial service activity is constitutive of social norms, religious beliefs and practices, and local cultures (Berger \& Udell, 2006; Avery \& Samolyk, 2004). Yet, not all SME finance is described as "relationship based" (Deyoung et. al, 2004). Thus, this section focuses on additional characteristics of community finance found within the literature and is designed to extend the SME finance and relationship banking findings highlighted in the afore section.

Neither community nor community finance is defined in a fixed format within the literature, even though sometimes practical designations rely primarily on a socially bound organizational size or geographical placement within a specific physical community (Archer, 2012; Delanty, 2010; Krannich et al., 2011; Sanders, 1966; Stone \& Nyaupane, 2014; Theodori \& Kyle, 2013) . Nevertheless, 
Deyoung et al. (2004) offer a flexible working description for community finance while simultaneously acknowledging its complexity:

A community bank is a financial institution that accepts deposits from and provides transaction services to local households and businesses, extends credit to local households and businesses, and uses the information it gleans in the course of providing these services as a comparative advantage over larger institutions. (p. 87)

Generally, a community bank is regarded as being embedded within a community: geographically (place), physically (brick/mortar on the street corner), and socio-culturally (involved with deep/active relationships). One of the tenets of community finance is its ability to do businesses with local businesses that require a symbiotic relationship with their financier(s) due to limited transactionlending portfolios. While community banks are largely focused on deposits and personal relationship lending, often at the local level, it is important to clarify SME finance is not regarded as only possible via small financial institutions or a niche strategy for larger firms (Deyoung et al., 2004). Consolidation of the finance industry has exerted pressure on community banks and the role of relationship lenders within organizations. Strategic positioning and personalized (even customized) service level management that gives the SME client access and knowledge on what he/she/they need for successful business operations are necessary for their continued viability when facing competitive threats from larger institutions. Furthermore, technology offers both a competitive threat and 
strategic opportunity for community banks (Deyoung et al., 2004; Stambaugh et al., 2020; Tolbert et. al, 2014).

Community banks, and their leadership, including relationship lenders (loan officers, etc.), emphasize their value via constructs of long-term relationships. They also seemingly elevate the importance of soft information in credit decisioning and have been found to participate in higher levels of risktaking as compared to larger financial institutions (Smulowitz et al., 2020; Uchida et al., 2012). These relationships require a critical balance, as community banks have been described as sometimes exhibiting "monopolistic power" while also being "vital" for local, community resilience (Tolbert et. al, 2014). Earlier work by Marquis and Lounsbury (2007) highlight a community logic framework for conceptualizing how community-level actors both shape and govern positionality; a critical point for conceptualizing how and why relationships between community financiers and SMEs are expansive with complex implications for social, ecological, and cultural norm developments (Marquis \& Lounsbury, 2007).

Community banks, similar to the SME enterprises and households they have historically serviced, are exhibiting evolutionary "pivots" as they co-innovate with SME clients for shared resilience and growth modeling, arguably practicing some level of "civic capitalism" as described by Tolbert et. al (2014), particularly if/when there is local ownership of the community bank (Tolbert et al., 2014). Individual persons and communities (e.g., natural resource-based communities) have long collaborated for reasons of common purpose, with hopes of positive outcomes for both human and non-human actors. In this vein, finance typically 
has an active, if not central, role in such endeavors. With increasingly observable patterns of growing interest and desired social-environmental activeness concerned with extreme poverty, climate risk, ocean health, as part of "everyday lives" (e.g., volunteerism, ESG investments), continued investigation into community finance's role and potential matters.

\section{Sustainable (and Social) Finance and CSR}

As finance has been described as the "grease" of socio-economic processes, a research agenda that explores such 'reach' arguably presents an opportunity to unpack how responses to sustainability initiatives may be contagious across industries and beyond organizational networks. This is particularly interesting as part of any attempt to understand successes and failures in achieving CSR objectives that have been linked to the Sustainable Development Goals (Dörry \& Schulz, 2018; Scholtens, 2006; Zimmermann, 2019). Similar to other industries, finance's business case for CSR is embedded within numerous operational functions that are encapsulated within strategies, planning processes, programs, structures, and systems. In finance, peer to peer pressure for CSR implementations is observable (Epstein \& Roy, 2001; Yang et al., 2017). Large-firm actors involved with such processes are likely affected by a multitude of exposures to the topic of sustainability while engaging in professional duties. Thus, what emerges is an approach to CSR that is not contradictory to the classic construct of firms' profit-first agendas, but rather aligns well to its positioning, competitive advantages, and strategic re-investment framework for both near-term and longer-range benefits and identities 
(McWilliams et al., 2006). Considering sustainable and social finance pursuits most likely arose from earlier CSR initiatives of other industries, it is important to consider if it is unfolding in way that best align to its purpose(s).ways. A recent bibliometric analysis by Secinaro et al. (2020) illustrates the orientation of sustainable finance, to date. The authors' analysis and findings include the following areas of emphases: human well-being and technological impact, governance, and topical convergence of ethical investment and sustainable development. This prompts a thoughtful reflection on what the traction thus far suggests. as well as what is and is not in the emerging academic library. In the article, entrepreneurship is the most referenced term while community shares a bottom ranking of the top 20 terms with energy, business, and care (Secinaro et al., 2021). If finance has unique or special obligations in terms of the SDG framework, in particular as it relates to partnerships, community-level salience, and long-range socio-cultural viability for ecological prioritizations, its orientation and measurement principles should go beyond that of other industries due to its levered positioning and power. As an example, what happens to the sustainable finance system design if/when the magnitude of support for entrepreneurship and community, respectively, are reversed? Does finance become more sustainable as a result?

A recent article by Migliorelli (2021) offers a concise approach to consider, making the case for an action-oriented frame for finance's role as "finance for sustainability." He argues sustainable finance should be, "Finance to support sectors or activities that contribute to the achievement of, or the improvement in, 
at least one of the relevant sustainability dimensions", while Fatemi \& Fooladi (2019) posit a sustainable value creation framework should be inclusive of all social and environmental costs and benefits (Migliorelli, 2021; Fatemi \& Fooladi, 2019). Calls for finance research focused on social inequity, informal exchange outcomes, value orientations, and social networks aligns well with sustainable finance's stated objectives, yet much of the literature to date primarily consists of critiques around practice/assessment, including a narrow focus, credibility issues, and time-horizon limitations (Carruthers \& Kim, 2011; Quatrini, 2021).

Existing literature supports the notion of collective interest and response, such as shared pro-environmentalism behaviors and ESG investment interests; however, there is a gap in scholarship for deep contextual analysis of financial firms' positionality communities and businesses that are co-navigating sustainability transitions, in both operationally routine and distressed situations (Singer, 2009; Archer, 2012; Humphreys et al., 2019; Salzmann, 2013; Singer, 2016; Urban \& Wójcik, 2019). Notably, there are selective uses of the terms "community" and "values" within the relationship banking, sustainable finance, corporate social responsibility, and sustainable development literatures; however, this conceptual pathway requires more of an investigative response to address how community, particularly when framed as participatory action such as community level finance, is present (Humphreys et al., 2019; Nájera-Sánchez, 2020; Smulowitz et al., 2020). 


\section{Community Interactional Theory and Pragmatic Landscapes of Sustainable Development}

Levinson proposes we conceive interactive capacity as a type of engine that is both universal and innate across humanity with properties of instinct, adaptability, analytical capabilities, motivation, communication (language-based and other), and temporal reaction embedded within (Levinson, 2006). This is in line with Jensen and Meckling (1976; 1994) who remind us of sociological perspectives of human behavior as direct products of cultural environments, inclusive of norms, traditions, and even choice constraints, all of which are action oriented or interactional in nature (Jensen \& Meckling, 1994). At the community level, interactional capacity has been characterized in the literature as a continuous process of interaction that is natural, authentic, continuous, and that directly shapes both individual and communal identities (Bridger \& Luloff, 1999, 2001; Flaherty \& Cope, 2016). For Wilkinson, "Community....is a natural disposition among people who interact with one another on various matters that comprise a common life (Wilkinson, 1999, pp. 16-17). Wilkinson also states that, "Community implies all types of relations among people, and if interaction is suppressed, community is limited" (Wilkinson, 1999, p. 17). In addition, both Kaufmann and Wilkinson conducted seminal works on the establishment of the community field as a complex of interactions between various actors and various temporal processes (Kaufmann, 1959; Wilkinson, 1999; Flaherty \& Cope, 2016). At times, the concept of community fields has been criticized for its fuzziness. Do they represent systems, processes, an organic wholeness, including the 
phenomenological experience of individuals (Flaherty \& Cope, 2016; Theodori et. al, 2020; Kaufmann \& Wilkinson, 1970)? Regardless, there is persistent theoretical debate concerning the boundaries and reach of community both by definition and structuration that most likely will persist. Nevertheless, community fields as described by Wilkinson (1999) are "a process of interrelated actions" (p.2) and offered this research project a perspective from which to launch inquiries into how SMEs are interacting and potentially affected by 1) the interactional capacity of the SME/financier relationship, 2) community-level interactional associations, if any, and 3) a conceptual approach for sustainable finance research that centers on contextual aspects of individual and community level interactional processes (field) across a landscape of shared informationexchange processes of financial intermediation that may inform knowledge transfer and meaning formation on non-financial subject matter (e.g., the Sustainable Development Goals).

The incorporation of landscape theory into the conceptual pathways of sustainable development and community interactional capacity expansions tied to sustainability is not unique to this project. Greider and Garkovich (2010) explicitly point to the social construction of groups' meaning formations and relationships with the natural environment, grounding their framework in phenomenology and symbolic interactionism while highlighting the group's identity as a group (think community) is self-defined via contextual selection. Seminal work on landscape theory can be attributed to Axelrod and Bennet (1993); they incorporated principles of organization into a structure of predictive 
aggregations of alignment by actors engaged in a situation defined by decisions, action, and navigation (Greider \& Garkovich, 2010; Axelrod \& Bennet, 1993).

Bridger and Luloff (1999) offer an interactional approach to sustainable community development, an approach that was later advanced by Mainzer and Luloff (2017) to conceptualize the field of pro-environmental behavior within fields (Bridger and Luloff, 1999; Mainzer and Luloff, 2017). Communities represent a shared identity as business owners, mutual interests, specific localities, shared experiences, or a combination of these attributes (Delanty, 2010; Wilkinson, 1999). Communities are embedded within the broader landscape of human experiences and networked habit formations that contribute to the social structure and dynamics of the landscape(s) they are placed in, associated with, or even indirectly connected to. The community interaction literature suggests community landscapes involve interdependent conscious and unconscious interactions that may be random, occasional, periodic, or continuous (Kaufman, 1959; Mainzer \& Luloff, 2017; Sanders, 1966; Wilkinson, 1999). A community landscape that encompasses the work network potentially becomes a community-in-work, thereby co-creating the community interactional landscape of sustainability linked attitude formations in business-first relationships. This can have far-reaching implications, particularly in settings where business life actively crosses other aspects of daily life as is the case in small U.S. towns. As pointed out by Zipin et al.'s (2012) re-imagining of community..."communities are thus 'not thing-like products but living processes wherein socially interactive and communicative people [continually] (re)create things and practices, and inject 
them with sense and meaning" (p.182). This seems to be a very relevant demonstration of the imperatives for contextual exploration from the viewpoint of community interactional landscapes. Simply put, the community lens proves an orientation for conceptualizing what is happening, holistically, across the visible and experiential landscape. Larson et al. (2015) argue the community landscape can be utilized as a mechanism for transcending well-being at the individual and on socio-ecological planes (Larson et al., 2015). Similarly, other scholarly articles directly link the community landscape to pro-environmental behavior, including volunteerism (Mainzer \& Luloff, 2017). Relatedly, research focusing on organizational commitment to CSR activities suggests connections between the aspects of a characterized community engagement with social capital and trust formations, employees' external support of CSR, brand and individual identities, knowledge extensions, and performance (Klassen \& Vereecke, 2012; Kuo \& Feng, 2013; Ng et. al 2018; Mizruchi \& Fein, 1999).

Individuals involved with community finance are doing so in socially constructed places, regardless of the interactive format or capacity. Through the lens of community as a landscape of experience, it is the objective to uncover interpretations of community finance and sustainable finance experiences as perceived during normal times, as well as if there is any perception or behavior change associated with a high risk or crisis period, such as the COVID19 pandemic. Largely, this is a question about both individual and community-level capacity for learning, knowing, and finding meaning in outcomes coupled with agency. Investigating the community interaction landscape of 
financing experiences offers flexibility for exploring community finance and sustainable finance elements that may be present for a specific locality or grouped identity, the levels of involvement by groups, any influences of external forces of the over-arching narrative, and how individual and community actions may be organized as it relates to finance (Aguinis \& Glavas, 2019; Balfour \& Alter, 2016; Bolis et al., 2014; Kaufman, 1959; Lauesen, 2016; Wilkinson, 1999). Facilitative processes of interactive capacity of persons, places, and shared identity groups offer significant insights into the navigational landscape of sustainability salience. Arguably, such an approach highlights the relevance of literature that place community as a social structure for those who seek societal improvements for all. From the perspective of the SDGs, a lens of interactive capacity linked to financial service interactions - community and individual businesses, regardless of business size, geographical placement, or sociodemographic characteristics - brings the discourse into the real (social and biophysical) world.

Classical pragmatism was also incorporated into the researcher's conceptual epistemology and reflective process. The key facets of pragmatism include: 1) ideas; 2) habit, belief, and disposition formations; 3) treatment of logic or reason; 4) role of language; 5) focus on experiences; and 6)an openness requirement for inquiry (Haack, 2006). Organizational networks engaged in CSR are experiencing repeated interactional patterns specific to organizational and/or community fields as described by Wilkinson, or webs, as characterized by Simmel (1908) (Schermer \& Dary, 2018; Coser, 2004; Wilkinson, 1984). 
Conceptually, pragmatism affords an openness for discoveries pertaining to the interactional environment and interactional outcomes' permeability into both the SME leader and collective meanings, habits, and assumptions that form both inside and exterior to community finance organizations. By incorporating the essence of the field, the web of the horizon, and the act(s) or activeness that is part of the interactive capacity that supports the conveyance of meaning. This latter point represents both the intentional and unintentional formations, as well as both active and passive information exchanges. Habit formations and communications are developed in such a landscape of interaction; shaping the individual and social experience of an experiential landscape, thereby situating the context for both the individual person, whomever they interact with, as well as possibly affecting the biophysical environment. What results is an organic outcome of habits, meanings, and possible meaningfulness formation that can result in value placements, all of which are permeable from interaction to interaction, from SME to SME, from household to household, and across a multitude of networks, all of which shape the landscape of experiential meaning. As a comparative example, CSR programming is often measured in its static forms, yet it is not commonly presented as "socially real". However, are the actions, attitudes and communication patterns not firmly embedded within the community and/or networks, directly affecting what does and/or does not get actioned? Arguably, even if something CSR related is communicated or part of the measurement design, ideas and beliefs emerging and being shared as part of social exchanges which occur when CSR programs are operationalized. There is 
a pragmatic effect; habits, beliefs, and future orientations that can grossly impact what comes next (Bacon, 2012; Bernstein, 2010; Misak, 2016). This is the essence, and arguably importance of pragmatism in the conceptualization of interactive capacity as it relates to the SDGs and the programs linked to them (often labeled as CSR or ESG in industrial settings). Historically, there has been limited treatment of the likely and real permeability of sustainability salience (or lack of) across fields and networks, but this is morphing with increased collaborations and the increasing activity involving the SDGs.

Sustainability as an idea is not new; in fact, in practice it has been part of the human experience for most of our shared history. However, from a pragmatic lens, it is an idea that is garnering much attention in recent years as the biophysical environment is reaching its limits of what it can or will tolerate as part of human interactions. Theoretically combining community interaction, landscape orientations, and pragmatism is useful in the sustainability discourse, and particularly responds to scholarly calls for deeper understanding of contextual meaning related to sustainability, the SDGs, and other sustainable development frameworks, as it fosters inquiry into a very human aspect of systemic design and orientations concerning the topic, our beliefs, and how they (may) affect what we do.

\section{Synthesis \& Conclusion}

"While there is no one universal definition, CSR or sustainable finance can be defined as the provision of financial capital and risk management products and services in ways that promote or do not harm economic 
prosperity, ecological well-being and community wellbeing ....... Community-level engagement will also be common, where FI collaborations with community leaders will be identifying win-win opportunities and their leverage potential. Sometimes these engagements will be advice-seeking, other times partnership-oriented, with the intent of working together to achieve better outcomes." (Strandburg, 2005) Unpacking accounts of SMEs' financial service explores one very important aspect of daily life - how and if individuals are experiencing sustainable finance via community finance relationships, and whether or not meaningfulness for sustainability linked subject matter results. Arguably, this study is an appropriate response to Strandburg's and others' calls for communitylevel engagements to connect the sustainable finance narrative in applied settings (Strandburg, 2005; Balfour \& Alter, 2016; Hemingway, 2019; Mainzer \& Luloff, 2017; Wilkinson, 1984). Furthermore, it offers an appropriate permeability of sustainability salience as a possible, future habit of the mind if consistently developed basis interactions with the idea of the SDGs can be incorporated into the sustained processes of community finance. As knowledge and opinions do not cease to operate when individuals move from internal organizational fields (e.g., within an SME workplace) to other fields of interaction and networking, a lens emerges for considering socioemotional connectedness of actors to ecological and systemic impacts, as well as more embedded socio-cultural perspectives (Brammer et. al, 2007; Ng et. al, 2018). 
Investigating for a pragmatic landscape of SME community finance as coexistent with emerging identities of sustainable finance, complementarily offers an emphasis on the criticality of individualized habits. Treatments of conduct as social and how innate impulses (e.g., fear) consistently shape habits, that on a cumulative basis yield functional dispositions resulting in certain conduct, are important contributions. Similar to Peirce (1878), it is in the course of the cycle that new discovery is possible. Dewey's (1922) central tenet of active reflection on the present in search of good (better vs. worse) conduct offers a practical lens for sustainable finance and community finance ideation and leadership (Haack; 2006; Misak; 2016; Valentinov \& Pies, 2018). From a perspective of reviewing large-scale CSR edicts and narratives, social conduct formation bridges the organizational culture literature by offering a vantage point for considering how and why CSR mindsets can and will take on completely different individual and both small/large group identities (Haack, 2006). Recent literature discusses social value transfer and systemic moral character as frameworks for CSR analyses at the firm level (Landrum \& Ohsowski, 2018; Valentinov \& Pies, 2018). These are applicable for firms of any size as well as for community financiers. Sociological and organizational literature both contain debates around socially constructed knowledge as well as conceptualizations of persistent counterdependencies between resources, with human capital (resource attributes and capacity development both) being a central theme in this discourse (Guerin, 1992). In this vein, micro-level investigations into SME and financier network characteristics, particularly individualized and small group attitudinal formations, 
value transfers, pro-environmental behaviors, are all contextually engagements or dis-engagements, and are within the scope of this research (Hemingway, 2019; Mainzer \& Luloff, 2017; Rosen, 2018). Interestingly, Littig and Griebler (2005) argue that work is a key concept of social sustainability (Littig \& Griebler, 2005). In addition, SMEs are working in their livelihoods and financial relationship activities. Aguinis and Glavas (2019) highlight sense making and meaningfulness in work, and SMEs and their network connections but to the communities within which they operate.

As global calls for increasing engagement on the topic of sustainability are both rather persistent and often make a reference to the power and necessitation of community' responsiveness, it is important to uncover opportunities for meaningful engagement on the subject matter. Community finance consists of active engagements that may present opportunities for such topical engagements. Financial supportiveness is an acknowledged component of sustainable development. If within landscapes of interactive experiences, an opportunity for increased knowledge and information capacity developments across communities of all shapes and types can be tapped into as part of the broader sustainability agenda, a tremendous amount of knowledge may be exchangeable.

The meaningfulness of sustainability, community development, sustainable development, and social responsibility is an increasingly important facet of the broader sustainability narrative. Through a research agenda that first looks to better understand the lived experiences of the landscape of interaction 
as part of community finance dealings by SMEs, then future questions of meaning and truth-making permeabilities across communities of interest, place, and type may unfold. This is particularly important when considering the emerging sustainable finance narrative. This project offers theoretical contributions to the evolving discourses on sustainable finance, sustainable development, business ethics, corporate social responsibility, community, and economic sociology. 


\section{CHAPTER 4: Methodological Approach}

This chapter presents the study's research design, which includes a conceptual approach, interpretive framework, important clarifications regarding my role as the research instrument, and methodological procedures.

A phenomenological method was utilized to explore the essence of the SME leader or owner experience with financial service providers as indicated by their ongoing relationships. The investigation sought to explore meaningfulness of these interactions through conversations on individual livelihood pursuits, community impacts, sustainability, and financier relationships. A hermeneutic phenomenological framework facilitated a search for embodiments of the population of interest's lived experiences through an analysis of interpretive acts and situational constructions of community finance and sustainable finance knowledge and attitudes, as recalled by both SME leaders and financiers (Desjarlais \& Throop, 2011; Lauterbach, 2018; Vagle, 2018; Van Manen, 2016).

The study is designed to deliver beneficial insights pertaining to the essence of the community finance experience. Community finance and sustainable finance are related. Findings that offer characteristics of meaning or habit formations, descriptive experience details, and structural descriptions around what is happening in the SME landscape of experience are anticipated.

The study may contribute to future research agendas and CSR discourses on the criticality of micro-analysis at the individual, firm, and community level as a fundamental aspect of knowledge formation pertaining to sustainability attitudes and habits across networks and organizational fields. The goal is to provide 
beneficial insight into if and how community finance contributes to SME sustainability, community sustainability, perceptions of livelihood well-being, and the global sustainability agenda.

The researcher conducted a qualitative exploration into the lived experience or lifeworld of SME owners and leaders who are interacting with financiers and vice versa. These interactions, and their capacity, were viewed as the mechanism for observable relationship characteristics, information exchange, and developments of shared meanings - past, present, and future - as SME and financier engagements occur in a variety of formats, including face to face, telephonic, and digital communications (such as email and text). Dynamic landscapes of financial interaction offered the research setting for discovering the essence of what transpires when SMEs and financiers navigate their shared relationship and knowledge while engaging in community finance. Perception development and belief formations, behavioral changes, and newfound habits concerning a host of sustainability linked topics were observable within the landscape. Examples include technological adoption or environmental stewardship direction obtained from financial program incentives, the emergence of new applications such as a virtual assistant, and risk-mitigation interactions. Although this is not an all- encompassing list, it hopefully offers insightful references for how and why contextual understanding of the SME and banker shared experience was utilized to link community finance with sustainable finance and ultimately the SDGs. 
A critical driver of the research agenda is that financial service interactions sometimes take place in the name of community finance and/or sustainable finance. So, as a priority, I wanted to first go under the surface of such labels and understand what the labels mean, if anything, to SMEs and financiers, particularly as reflected upon in the context of interactive experiences. Exploring such experiences of small-medium business owners and their respective financiers offers an opportunity for comparing them to the narratives of community finance and sustainable finance. Ultimately, it is my hope that any research findings associated with this study will contribute to a better understanding of what community finance and sustainable finance mean to individuals and communities today, as well as their meaningfulness (current and prospective) to society as we collectively work to navigate the changing demands of human-nature environments.

\section{Why a Phenomenological Inquiry?...The Lived Experience Says Alot}

I selected the phenomenological philosophy and methodology to explore

SME and community banker lived experiences for a couple reasons, but primarily due to my past professional experience as a financier and current co-ownership status as a SME leader. As background, researchers engaged in psychological, nursing, and anthropological research have used the current method, as have many other human science disciplines focused on human behaviors and emotional experiences. The foundations of phenomenology, both in terms of philosophical underpinnings and methodological characteristics, originate with the works of Husserl (1922) and Heidegger (1927). It has continued its 
epistemological metamorphosis through the scholarship of social sciences across numerous disciplines. There is no absolute right way to do phenomenology as its usefulness now traverses specialized applications for unpacking the essence of experiential phenomena ranging from religious to political to technology encounters. As both a philosophy and a methodology, phenomenology is human-centered, necessitates attitudes of openness on part of the researcher, incorporates deductive and inductive practices of spiralized data analyses that support the co-occurrence of epoche (bracketing), thematic discovery, and language constructs for communication of observed meanings (Bevan, 2014; Caelli, 2000; Desjarlais \& Throop, 2011; Englander, 2012; Finlay, 2014; Hoffding \& Martiny, 2016; Johnson, 2012; Laverty, 2003; Moustakas, 1994; Vagle, 2018; van Manen, 2016). According to Van Manen (2016), “....phenomenological interest lies in understanding the meaning structure of a phenomenon that makes it meaningful and distinguishable....", thus this is the rationale for its distinctiveness as both a philosophy and methodology (van Manen, 2016). As a methodological discipline, it has been nicely summarized in the Stanford Encyclopedia of Philosophy as:

Phenomenology is the study of structures of consciousness as experienced from the first-person point of view. The central structure of an experience is its intentionality, its being directed toward something, as it is an experience of or about some object. An experience is directed toward an object by virtue of its content or meaning (which represents the object) together with appropriate enabling conditions. (Smith, 2018 - Stanford) 
The discipline of phenomenological inquiry as a methodology has a variety of categorizations that range from descriptive, interpretive (hermeneutical), transcendental, gendered, as well as a variety of other frames of specialized interest, and, as a result, it is important to clarify the characteristics of the selected approach. This is to maintain coherence across the study design (Finlay, 2014; Vagle, 2018). There were important elements of inclusion that were necessary to highlight concerning the selected methodological approach. Examples include how the researcher had to position herself, the epoche approach (bracketing), approach to designing the interview, reflectivity in interpretive processes, and which lenses to select when exploring concepts, as well as how the researcher used the literature on the topic of interest (Merriam \& Tisdell, 2016).

Community finance, conceptualized as a community field of interaction offered a robust framework for phenomenological explorations, as it is very human-centric. In addition, seeking to understand how small-medium business owners (SMEs) are experiencing community finance and sustainable finance as part of their overall interactive experience with their community banks offered an opportune context for seeking essence of community finance and its relational attributes to sustainable finance. In addition, due to the recent COVID19 pandemic, the topic became more urgent as conditions of routine business were disrupted for small businesses and millions of others (Wilmoth, 2021). Furthermore, as both academic and trade literature present inferred connections between community action and sustainability outcomes, my research interest has 
long been wrapped around this curiosity, making it prudent to investigate for real and/or fictitious alliances between the two. Talk can be quite cheap and dangerous when it generates confusion, disappointment, or the combination of the two. Community finance, as an experience, has been instrumental for my personal livelihood, a fact highlighted by the recent pandemic. Furthermore, sustainable finance has so much potential, thus it should be realized in its most optimal design. As a researcher, my lingering questions on how community finance, sustainable finance, and the SDGs are all connected, or not, is what motivates me.

My purposeful objective for this phenomenological study on SME and financier interactive experiences is to eventually advance theorization and applied policies around the interactive capacity of community finance, and to explore its potential for aligned contributions to measurable progress linked to sustainable finance and to the broader sustainable development agenda. By phenomenologically investigating the dimensions of SME finance as a landscape of interactional experience, an exciting platform for clarifying the role (current and potential) of community finance its sustainable finance linkages to the collective SME footprint of sustainable progress emerges.

\section{Hermeneutics and Phenomenology}

Hermeneutical processes have long been applied to interpretive processes aimed at extracting structural characteristics of comprehension and meaning formations of a whole or big question. Generally, this is done through the investigation of the parts of evidence available for such interpretive action. 
This can often primarily consist of text analysis but also more broadly draws from all forms of subject-object transfers of conscious and unconscious meaning found in shared language and observable actions. Hermeneutical structures are abstract in nature but arguably no less important than if a measurable physicalness was present. Furthermore, they are considered as having been socially constructed as having a higher purpose which is what largely justifies the method (Lauterbach, 2018; Laverty, 2003).

The interactive processes between the researcher and the study participants characterizes hermeneutical phenomenology, with both language and observation as the units of analysis. Language data is usually collected from the first-person perspective through a semi-structured interview process, which is the approach used for this study. Hermeneutical phenomenology is interpretive in its nature and stems from Heidegger's philosophical belief that it is impossible for someone to fully bracket or to set aside what they already have experienced or know about a topic of interest (Peoples, 2021). My selection of this method was important as it afforded an open design to ultimately work toward thematic discovery via cyclical reflexivity and ideation processes around what I already knew, learned from the interviews, and what became creatively "possible" per the study's conceptual framework. A complete desire to unpack the essence of a phenomenon, as part of consciousness in daily life experiences, is a cornerstone of phenomenology (Vagle, 2018; Valentine et al., 2018; van Manen, 2016). As this nicely aligned to my purpose-oriented goals, it became clear it was the most appropriate fit for the study design. The hermeneutical approach, similar to other 
forms of phenomenological inquiry, incorporates repeated analytical processes of the data set to discover abstract themes. Nevertheless, it acknowledges my own prior formations of knowledge on the topic of interest and provides space for awareness of possible embedded prejudices that I worked through as part of the process. This, in Heidegger's works (1927), is referred to as the hermeneutic circle. Importantly, this is not a specific descriptive technique, but rather a guiding principle for the purpose of sense-making and understanding what is observed and captured in the data (Peoples, 2021). Phenomenology's comprehensiveness and flexibility are beneficial for highlighting and exploring initial and revisionist cycles of productive thought work.

Considering the vagueness that exists with definitional and descriptive approaches to community finance and sustainable finance (and relatedly SME finance), the phenomena of interest in the study, I determined that there was real benefit in applying the selected research methodology to explore current experiences of SMEs (including my own experience) engaged with financiers in their livelihood pursuits. Furthermore, my ongoing interest in how pragmatism and community interactional theories align to the phenomenological philosophy facilitated this methodological approach. From a pragmatic lens, conceptualizing how community-level phenomena are related to beliefs and habits at the individual, enterprise, and community levels makes space for acknowledging unintentional forces of influence on conscious experiences. As a result, a very early concept of pragmatic phenomenology is in early development. 


\section{Epoche/Bracketing}

Epoche is a distinctive element of the phenomenological research methodology. It is described as one's ability to refrain from judgement and is commonly referred to as "bracketing". Within hermeneutical phenomenology, researcher judgements are not fully diminished in the process, but rather incorporated into the discovery process through a repeated and conscious effort to acknowledge what is already understood and/or known by the researcher about the phenomena of interest. The goal of epoche is to ultimately facilitate a process that results in reflective and constructive observations and thematic developments about the phenomenon while giving space for pre-existing knowledge that may come from direct experience or other interactions, such as the literature review process. This is important, as it is often the case that research questions come from a place of interest due to personal experiences or observations. This was the case in my study. Most likely, I would not have developed the research questions on how community finance and sustainable finance are related in the eyes of SMEs if: 1) I was not personally a SME, 2) I had not previously worked in the financial services industry, and 3) I had not been actively engaging with a community bank to navigate the enterprise level sustainability of my family's company. In essence, I was consciously experiencing community finance as part of both normal and crisis navigations. As a result, the questions and data collection process (semi-structured interview and narrative text observations) were informed by my pre-existing knowledge. Thus, in accordance with the epoche requirements, I had to work diligently to 
hold aside any personal prejudices, viewpoints, and assumptions throughout the various study phases. My experience was not their experience, and vice versa, yet all of it was important to the process (Merriam \& Tisdell, 2016).

\section{The Researcher's Role - As the Instrument and Navigating Epoche}

A phenomenological researcher is considered to be the primary research instrument of this type of study (Peoples, 2021; Van Manen, 2014). Due to personal experience as a business owner who deals with financiers for my own household livelihood, as well as having worked within the financial sector for several years, it was important that I consistently revisited my personal orientations, attitude, and possible biases throughout the various phases of the research project. Notably, the research questions were born out of direct experience of navigating relationships with banks, both large and small, during the early months of the COVID19 pandemic and when the PPP and other emergency lending programs were launched. Upon reflection, I realized my thoughts about community banks, specifically the question "What does that even mean?", became more active as part of individual response to the changing landscape. At the same time, my interest and knowledge about the global sustainability agenda and of the essence of corporate responsiveness, was not lessened in any way as a result of the pandemic. What resulted was the development of a second and related question, "What does sustainable finance even mean?" Furthermore, "What does sustainable finance mean, particularly in times of crisis and time sensitive risk management?" Drawing from earlier reviews of the literatures on community interaction, pragmatism, sustainability, 
and sustainable development, and corporate social responsibility (including ESG), I was already aware of the semi-active use of the word "community" as part of the emerging discourse on sustainable finance. Through a process of personal reflection about my own interactions with financiers in response to COVID19, an active research agenda focused on community finance and sustainable finance experiences and meaning formations, particularly for SMEs, in both normal operations and crises periods was formed.

As the primary researcher, I allowed my pre-existing knowledge and earlier literature review activities to guide the expansion of the initial research questions into a semi-structured interview format that would facilitate the interviewees' reflective processes. In order to achieve the aforementioned epoche/bracketing, a few minutes of orientating self-talk prior to the interview sessions were conducted. This mostly included a review of the interview questions but also a simple "turn it on" engagement mentality, describable as going into "actor mode". Throughout the interview process, I took great care to not provide any details about pre-existing viewpoints or personal experiences about the topic of interest. For example, I did not share my SME business background with the interviewees. I also repeatedly redirected the conversation to the interviewed parties' perspectives and actively managed for my own "silence" when individuals asked about my thoughts. However, I did explain that both of our thoughts would be captured. during the interview and reflection processes. For the benefit of making the interviewees as comfortable as possible, I also expressed a very active interest in talking as much as possible 
after we covered the questions. This was found to be particularly helpful as many participants expressed interest in learning more about me, my research, and what I hope to do with the results of the study. Achieving a degree of sustained openness and flow in the data collection is of critical importance for a successful phenomenological study, thus I very intentionally worked to establish a space of information sharing.

It is important to note, the literature also does not present any fixed definitions for the phenomena of interest, another factor that contributed to selecting the phenomenological method to explore community finance and sustainable finance. As a result, I intentionally did not definitions of community, sustainability, community finance, or sustainable finance within the interview process. The rationale for no set, but rather flexible working definitions codeveloped via the dialogue, was to maintain as much openness as possible for the interviewees to describe their experiences in both their own words and frames of reference for what community, community finance, sustainability, and sustainable finance mean to them.

During the multiple phases of analysis, which included practices of phenomenological reduction, horizontalization, and imaginative variation, I mindfully challenged and checked personal thoughts and viewpoints, as they arose from time to time. This included maintaining awareness that community finance and sustainable finance are undefined. As needed for the integrity of my process, I made notes in a journal for future, deeper personal reflection and/or next generation research ideation. The development of themes was informed by 
my pre-existing knowledge. However, I actively set aside my own experiences as much as possible when interpreting the data. Themes that are attributable to both the "parts" (e.g., individual participant case) and the "whole" (e.g., an observably shared experience), were developed in accordance with the collected data findings, while acknowledging what was already known (by me, experientially).

As a final clarification point, I want to express that I do not consider myself to be overly emotionally charged (e.g., love them or hate them) about community finance and/or sustainable finance. In general, I maintain positive interactions and relationships with financiers of various size and profiles and truly want sustainable finance initiatives to yield positive social-ecological outcomes. I hope sustainable finance and the SDG framework both "work". In retrospect, I acknowledge it would have been much harder to separate my thoughts from the "interviewer actor" role in both the data collection and analytical phases of the project if this were not the case. However, my approach to epoche has arguably been proven successful, as measured by the real surprise I experienced with some of the study findings.

\section{Research Design}

Van Manen's $(2016 ; 2017)$ hermeneutical phenomenology approach was utilized in the study design. The central research theme focused on normative interactions that may contribute to meaning formation and/or knowledge sharing about community finance and sustainable finance. The population of interest includes both small-medium business owners or leaders (SMEs) and community level financiers engaging with the aforementioned population of interest. Four 
research questions were developed and relied on for the over-arching purpose and design, participant criteria and selection, and project operationalization. Below are the research questions:

1. How do SME leaders/owners experience 'community finance' and/or 'sustainable finance'? What does it mean to them?

2. How do financial sector actors engage with SMEs and does 'community finance' and/or 'sustainable finance' service and knowledge exchange occur?

3. Are publicly available narratives of 'community finance' and 'sustainable finance' related to their individual experiences?

4. What is the essence of the relationship between the narrative and the experience of the individuals participating in 'community finance' - in both 'normal' and 'crisis' operational frames?

\section{Procedures}

A phenomenological method was deployed to explore the experiences of SMEs and financiers as they interact with one another for the purpose of exploring socially constructed elements and outcomes of financial dealings within place-based and/or interest-based communities. The research questions were operationalized through the development of a semi-structured interview process to facilitate reflexive responses. The questions focused on the interviewees' interactive experiences, knowledge sources, associative meanings, habits, and personal definitions of community, sustainability, and community finance. Participants were also asked about their knowledge of sustainable finance and 
the Sustainable Development Goals. The population of interest includes U.S. based SMEs engaged with financiers as part of business and livelihood operations. The population (SMEs) utilizes financiers in a variety of ways to support business operations, all of which may offer useful insights to the research questions, so the data collection was open to any/all types of financial dealings but had to be specific to the needs/activities of the SME business.

\section{Participant Criteria (Sample)}

A sample population was purposefully identified to support the goals of the study. As there is no minimum count requirement for the number of interviews in a phenomenological study, a target of 25-40 interviews was initially set for purposeful representation. A criteria-based sampling (Creswell, 2013; 14) technique has been deployed to obtain interviewees for qualitative insights into experiences of SME owners and financing partners located in the Midwest region of the U.S. Participation in the study required business ownership or authoritative leadership, size qualifications in line with SBA thresholds for small-medium business designation in the SME's respective industry, and a geographic location in the U.S. Midwest. The latter criterion was to enable researcher access during the COVID19 pandemic, but it is important to note that the criteria-based design is suitable for a national scale research agenda. SMEs located in both urban and rural settings and across both goods and services industries were eligible for study participation. 


\section{Research Setting}

Due to geographic proximity and accessibility, the study was conducted in three midwestern states: Missouri, Nebraska, and Illinois. SMEs and financiers in both urban and rural settings within the states were engaged in the study. Urban participants were located in large metropolitan regions of the states. Rural participants were within one to two hours driving distance of those metropolitans. During the course of the study, it was also noticed that each of the locations is involved with distinct branches of the Federal Reserve banks, which may lend itself to further research opportunities on comparative program access.

\section{Recruitment}

Potential interviewees who met all the criteria of the sample population were approached directly via email or a phone call, or indirectly by a mutually known party. In the event of the latter, an introduction was made, followed by a direct request for research participation and consent. Upon request, brief summaries of the scope of interest were shared with interviewees who wanted to know more about the nature of the study prior to committing. All participants were made aware of mandated IRB procedures and confidentiality. Furthermore, interviewees were informed that there would be no direct questioning about personal financial status, earnings, or amounts of monies received by the business in normal or crisis periods of operation.

\section{Participant Details}

A total of 27 SME business owners and/or community bank leaders were interviewed for the study. Each of the twenty-four SME leaders was confirmed as 
the primary decision maker and interactive party involved with financial activities for the company. Each of the three financiers interviewed were confirmed as actively involved with SME financing. The demographics of the sample population included 17 men and 10 women. Of the 17 male participants, thirteen male participants self-identified as white, three self-identified as Black/African American, and one as Hispanic. Nine female participants self-identified as white and one self-identified as both white/Hispanic. The average age of the sample population was 53 years of age, with a participant age range from $40-80$ years of age. All study participants had a high school degree or higher, with the majority having a bachelor's degree or higher.

The average number of years the SME businesses had been active was 35 years, with a range of one to $100+$ (non-specific for privacy reasons). On average, the companies employed 44 persons. Importantly, as this is a study about the essence of individual and community-level experiences of community finance, I was also diligent about avoiding prescriptive demographics to make space for expression of self-identification related to professional experience and industrial knowledge levels. This flexibility also extended to designations of rural vs. urban identities associated with their businesses and communities. The reported rural/urban breakout of interviewed business locations was approximately $55 \%$ urban and $45 \%$ rural, with one SME identifying as being both. Industries represented in the study included healthcare, real estate, manufacturing, food service, food production (agricultural and manufacturing), 
distribution, consulting/professional services, social services, and financial services.

\section{Data Collection}

Semi-structured Interviews. Semi-structured interviews were conducted over a five-month period, beginning in September 2020 and ending in February 2021. Notably, all interviews were conducted during the COVID19 pandemic response period. Over the course of an approximate hour, study participants were prompted to reflect on their experiences and interactions as part of SME financial dealings. The interview questions opened with business and experiential generalities about financial interactions and moved in a guided direction toward topics of community, community finance, sustainability, and sustainable finance. The interviews were recorded for the iterative coding procedure to be actioned at a later point in time. Each interview was designed to occur in a single setting; however, participants were asked to be open to follow-up clarifications. Due to COVID19 safety protocols, meetings were either in person, in a socially distanced format, or virtual discussions via Zoom. Purposeful sampling was conducted to represent both rural and urban communities. Post-collection, the interview data was organized, described, and aggregated into thematic categories via an iterative, interpretive process.

\section{Analytical Design}

The analytical design was a qualitative, phenomenological analysis semistructured interview data from the sample population of SME leaders and financiers. The process was iterative, reflexive, reductionist, creative, 
imaginative, constructive, and involved multiple phases specific to hermeneutic phenomenology: epoche and horizontalization of the data to equalize the initial stage analysis, as well as repetitive cycles of ideation around the essence(s) of the phenomena (Creswell, 2013; Merriam \& Tisdell, 2016). Central to the analysis was an approach to thematic coding that was open yet guided by literature and personal experience at the initial stage. The study's rationale was also supported by guided theoretical reflections on the epistemological and ontological characteristics of community, community finance, and sustainable finance as interactional processes with an assumption that such processes are socially constructed and inextricably linked.

According to Merriam and Tisdall (2016), "The aim is to arrive at structural descriptions of an experience, the underlying and precipitating factors that account for what is being experienced" (Merriam \& Tisdell, 2016). As they clearly state, this is the objective of the analytical approach to this study.

\section{Data Operationalization}

The data operationalization and analytical process incorporated a modified hermeneutic circle, or loop, that emphasized the qualitative aspects of the collected data (e.g., the interview text) that presented contextual interaction details, inclusive of content of important statements, evidence of meaning, habits, and belief formations on the topics of interest, and specifically community finance and sustainable finance experiential associations. The objective was to search for perceivable attributes of emergent experiential consciousness and essential meaning from observed interactive capacity regarding these topics. The iterative 
reflective process was guided by the diagram below. This approach served two purposes: 1) to support an appropriate epoche/bracketing procedure, and 2) to support my creative and imaginative processes of discovery for evidence of meaning(s) formation.

Figure 2. Phenomenological Analysis Approach

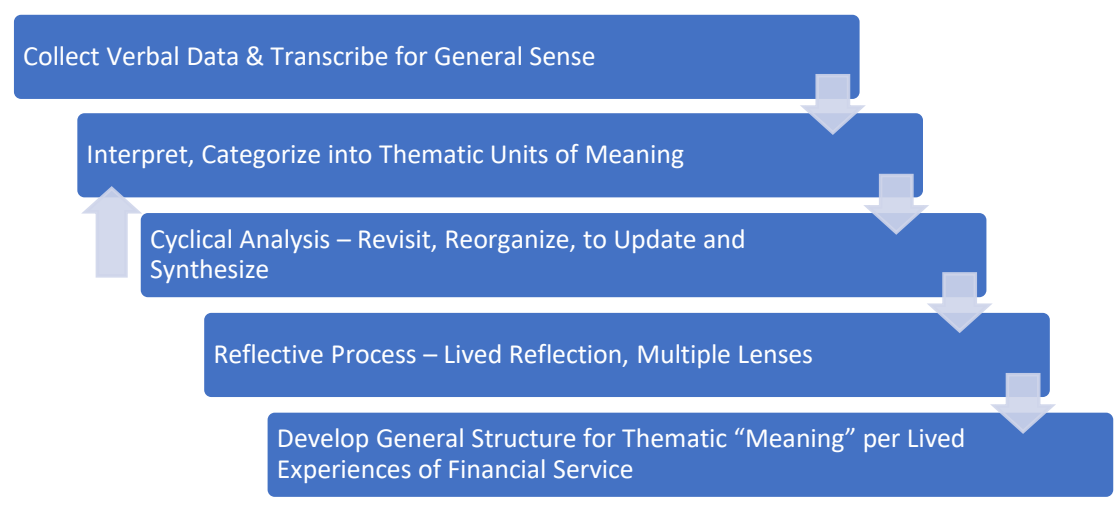

Adapted from Peoples, 2021

\section{Theme Development}

Themes were developed and revised throughout the analytical process, ranging from openly coded observations to deeper assessments of what was later found in incremental reflections. Selected themes were required to meet criteria of question sensitivity and conceptual alignment across each phase of thematic developments (Merriam \& Tisdell, 2016). Relational aspects of the emergent themes were also reflected upon and closely compared to the research questions and conceptualized in terms of both their uniqueness as part of the experience as well as linkages to the wholeness of an experiential essence. Social constructivist perspectives, pragmatism, landscape theory, community 
interactional, and sustainable development lenses were drawn upon in various stages of conceptual analyses.

\section{Verification of Findings}

Fundamentally, validation in qualitative research relies on research grounded in ethical conduct. Phenomenological research's validity and reliability are dependent upon an ethical approach that meets the assumptions of the philosophical perspective of what a phenomenology "is" as an inquiry and reliable documentation of experiences in everyday life. As a subjective research design, it is imperative that the data and researcher be credible resources on the topic of interest; that there is transferability of the collection and analyses processes; and that the findings are dependable, confirmable, and offer a potential for generalizable understanding.

As the purpose of phenomenological inquiry is to identify abstractions that suggest the essence of a phenomenon, thick descriptions are utilized to express the findings. Thick descriptions are important for offering supportive details for meaningful discourses and reflection on behalf of the audience to connect how various findings may relate to the holistic observations. In this study, the findings and discussion are supplemented with thick descriptions directly from the data as well as per my conceptual thought work to allow for verification of thematic discoveries concerning knowledge, observed beliefs or habits, and meaning and meaningfulness of community finance and sustainable finance. 


\section{CHAPTER 5: FINDINGS}

This chapter presents the research results and also provides an organized, thematic presentation of findings that correspond with the study's research questions. The study involved a multi-phased data collection, analysis, and eventual reduction that culminated in the emergence of discovered themes in response to the research scope, design, and emergent conceptual framework (Bloomberg \& Volpe, 2019). The quotes utilized in the presentation of findings are not unique to their respective placements and are representative "parts" of the "whole" essence of observational findings. For the hopeful benefit of a thoughtful reflective process on behalf of those who read this study, and in alignment with the cyclical and multi-lensed analysis that was conducted to highlight the lived experience of community finance, the findings have been organized to build from an individualistic perspective of SME experiences, relationships, and knowledge formations to more complex and abstract considerations of relational power and other influential aspects of community finance and sustainable finance. The latter attempts to search and identify meaningfulness, intentional and unintentional habits, and the overall essences and potential connectedness of community and sustainable finance. The intention of such an organized construct is to prompt an awareness of SME and community bank interactions in varying contexts and their effects (and potential effectiveness) across community landscapes. The big question (or central investigative frame) remains steadfast in the presentation of my findings: What is 
community finance and/or sustainable finance to small-medium business owners and community-level financiers?

\section{Setting and Population Representation}

The study was conducted in urban and rural settings in Illinois, Missouri, and Nebraska. The criterion-based sample is representative of SME demographics across the population of interest and U.S. based SMEs, particularly those located in the midwestern part of the United States. Industries represented in the study include healthcare, real estate, non-food industrial distribution and manufacturing, food production (agriculture, processing, and packing) and food service (retail), consumer goods sales and service, and social and financial services. The rural/urban designations of interviewed SMEs were 14 urban, 12 rural, and one that that self-described as being both urban and rural, resulting in an over-sampling of rural based business as compared to the $17 \%$ of the SME population that is counted as rural, as indicated by the most recent United States Census (advocacy.sba.gov, 2020). The U.S. Census classifies urban and rural as follows:

The Census Bureau's urban-rural classification is fundamentally a delineation of geographical areas, identifying both individual urban areas and the rural areas of the nation. The Census Bureau's urban areas represent densely developed territory, and encompass residential, commercial, and other non-residential urban land uses. The Census Bureau delineates urban areas after each decennial census by applying 
specified criteria to decennial census and other data (advocacy.sba.gov, 2020).

In addition, the U.S. Census Bureau identifies two types of urban areas:

- Urbanized Areas (UAs) of 50,000 or more people, and

- Urban Clusters (UCs) of at least 2,500 and less than 50,000 people.

And, "rural" encompasses all population, housing, and territory not included within an urban area (Census.gov, 2020).

However, the study design did not solely rely on this classification approach since hermeneutical phenomenology is grounded in a philosophical approach that relies on discovery of meaning through language, expressions, reflection, and open dialogue rather than overt influences that may form due to definitional constraints. For this reason, I asked the participants to identify the urban/rural characteristic of their SME operation from their own perspectives. Furthermore, my interest in rural business operations is in some ways outsized compared to more urban settings, primarily as it relates to the role of community banks in rural perceptions and behaviors on sustainable finance and meaning formations around the topic of sustainability. This is due to both my personal and professional backgrounds, and since I was raised in a small town, have owned businesses in both small towns and cities, for which I work (or in some instances have worked) closely with community banks in small towns to navigate my SME's operational needs and strategies. 
Nevertheless, it is important to note that it is estimated rural and urban businesses exhibit very similar rates of revenue and profitability, with some data pointing to slightly better rates of profitability for rural businesses vs. urban based companies (score.org, 2021; fundera.com, 2021). Also, urban and rural businesses indicate similar reasons for failure as well as ongoing operational challenges and factors of importance, such as lack of demand, lack of labor quality, and capital access. Experiential interactions between SMEs and their financiers, regardless of any actor's geographical location, is the primary factor of interest and given the similarities in economic opportunities and challenges, I determined it was appropriate to explore both urban and rural community finance processes in a similar frame.

Additionally, the COVID19 pandemic offered a unique opportunity for the deployment timing of the study to include comparative frames of reference between normal business operations and what occurs in periods of crisis. Unpacking perceptions and behaviors of SME leaders and the relationships they have with their financiers while navigating COVID19 responses offers beneficial early insight into community finance as part of SME risk management. At the time of data collection and analysis, SMEs and financiers were in a situation of co-navigating responses to a socio-economic event that had no similar magnitude within recent decades. I was curious to investigate how pandemic responses were being perceived, what the role of the community financier and SME relationship may be (if any), and any notable change effects the pandemic may be having on SME and community banker relationship dynamics. For this 
reason, questions were asked about levels of communication activity between

SMEs and community banks, observed implications of technological change, and cultural habits related to the ongoing relationships of SMEs and financiers (community or others) in comparative frames recalled as "normal" or "typical" (before the pandemic) and during the pandemic response, or "crisis" period.

\section{Participant Demographics}

Study participants included a total of 27 SME business leaders and financiers, consisting of 17 men and 10 women who identified themselves as either Black/African-American, white, or white/Hispanic. The average age of the sample population was 53 years of age, with a participant age range of $40-80$ years. All study participants had a high school degree or higher, with the majority having a bachelor's degree or higher. The average number of years the involved SME businesses had been active was 35 , with a range of one to $100+$ (nonspecific for privacy reasons). In comparison, the SBA data on small-medium businesses operations in the U.S. as of $2020^{*}$ reporting ("draws from BLS data from 2016-2019 period) indicates that of the approximated 31.7 million small businesses in the country, $21 \%$ of SME businesses with employees had at least majority-female ownership. The inclusion of female-owned businesses without employees (sole proprietorships, single-member LLCs, etc.) increases the estimated number of female-owned SMEs to 9.9 million, representing approximately $31 \%$ of all SMEs. Interestingly, Missouri had one of the highest \%s

of female-owned businesses as compared to other states. As of 2020 , non-white SME ownership was reported as approximately $16 \%$ of SMEs operating in the 
United States (advocacy.sba.gov, 2020; Highfill et. al, 2020). As of $2020,84 \%$ of all SMEs were owned by persons over 35+ years in age. In addition, while the often cited "most small businesses fail within their first year" does apply from select perspectives, some statistical indications support an approximated $50 \%$ survival rate for five years or longer, with a third of businesses surviving in excess of 10 years (advocacy.sba.gov, 2020).

The research design required an openness toward definitions of words pertaining to the topics of interest. This is both in line with the existing literatures on sustainable finance and community finance, as well as my own observations in both academic literatures and trade communications. There is not yet a single, agreed definition for community, community finance, or sustainable finance, and perhaps such a standardization would do more harm than good. Regardless, no fixed or formal definitions were provided for the interviewees. They were asked to reflect on their experiences with their financiers and vice versa as part of their own constructs of meanings for community, sustainability. My priority was to collect interviewees' own thoughts on what community finance and sustainable finance represent.

\section{Development of Findings and Situated Narratives}

A critical benefit of the phenomenological methodology is its usefulness in extracting observational "so whats" around reflective processes and interpretive responses in contextualized situations and dialogues (Norton \& Sliep, 2018). This was found to be true in the course of my interactions with the study participants, namely their own observably reflective processes as we discussed what was 
happening in their community finance processes. Excitingly, this conversation sometimes extended to what was also possible in the future. The latter is important to both the findings and conclusions of this research project.

\section{Summary Introduction to Findings}

The majority of the small-medium enterprise owners/leaders who participated in the study reported some level of dependency upon their financial resource partners. The range of involvement was broad, with a few indicating very low levels of needed support and a few expressing levels of immediate needs for critical support for ongoing business operations. The low-need SMEs generally are self-financed at present, carrying no to little debt obligation and primarily utilize financiers for operational processing (e.g., deposits, basic operating accounts) or in the event of major strategic expansion. The higherneed SMEs indicated much deeper involvement with their respective financial service providers, including facilitations of both short-term and long-term loans, equipment financing, working capital requirements, risk advisory and products, and, at times, strategic navigational support with day-to-day decisioning. However, the majority of SMEs participating in the study are characterizable as having moderate, routine, levels of financier activity as part of normal business operations, which can be generalized as operational support and limited or periodic debt or capital financing. The findings suggest an observable trend of increased numbers of financial partners as the size of the SME increases, with most of the sole proprietors dealing with a single bank or financier, and the larger SMEs maintaining accounts with more than a single financial enterprise and in a 
combination of size and profile frameworks (e.g., a national bank plus a community bank).

All SME owners and leaders that were interviewed indicated having an increased level of dialogue with either their bankers and/or accountants due to the COVID19 pandemic and its potential effects (real, perceived, anticipated, etc.) on their businesses. Outreach by financiers was quite variable as evidenced by differences in subject matter content (information richness), discussion context and tone, perceived knowledge presence in the exchange, and proactive vs. reactive communications modalities. Regarding both normal business and crisis period financial interactions, the majority of SMEs interviewed expressed quite positive dynamics and relationships with bankers whom they considered to be community-oriented financiers. The size and scale of these financiers ranged from small, single branch banks to large, regional banks (in some cases, multistate operators). Furthermore, positive dynamics were reported by SMEs involved with more specialized financial service enterprises, such as agricultural finance cooperatives and equipment financiers. A couple of SMEs involved with the study also reported having positive experiences with de novo banks, via $100 \%$ remote experiences via fintech enterprise relationships. 


\section{Findings Grouped By Research Question}

\section{Q1. How are SME leaders/owners experiencing 'community finance' and}

‘sustainable finance'?

Finding 1 - The lived experience of SME finance involves trust, trauma, and possibly everything in between. SME business owners seem to really like their financiers, especially those who have stuck with them in hard times. When asked directly to describe whether relationships with financiers were positive, negative, or neutral, the overwhelming frequency of responses was that the relationships were quite positive, and a majority of SMEs had maintained relationships with their financiers for several years as a result. SMEs reported sticking with banks for a few reasons, but primarily either because of the bank's conduct in a community setting or because of bank personnel with whom they had developed lengthy relationships. In general, the study participants indicated at least some degree of "stickiness" concerning the interpersonal relationship and the perceivably shared community dynamic of the relationship; a finding that is in line with much of the scholarship on relationship banking.

Most of my funding, as came from as far as loans and stuff is came from small community banks, that I can build relationship with that had a vested interest in improving the quality of life in that community. Okay. And so we really, really share the vision with the banks, to them know what we're going to do. And then we talked about the profitability of it, and the sustainability of it, okay. ...So that's how we have at managing and even in some areas, we have created employment opportunities or income opportunities by providing services.... Okay. So those are kind of the ways we have but we have basically, we're really work with small local community banks, we have a vested interest in their stake in the community." (Male Participant, aged 45) 
Exceptions to both "likeability" and relationship longevity were observed, specifically via discussions with healthcare and food industry related participants who indicated recent changes to financier relationships. The drivers of such change included a "forced response" due to COVID19 policy modifications by a national financial institution, a "lack of" product/service availability for financing a long-range risk management strategy, a "growth opportunity" for expansion and acquisition scenarios, a perceived "dirty dealing" (e.g., unethical activity with conflicts of interest present), and needing "greater understanding" of short-term business needs. Notably, a couple interviewees conducted rapid financier changes perceived as prompted by the pandemic, yet others indicated that relationship changes (past, present, or future) were more linked to ongoing business strategy, operations, and price-shopping of rates, as well as community-level dynamics between parties that had long-term relationships:

So for our facility, we utilize a lot of banks, okay. So you know, as in being in a small community, we try and support all of the local banks to some degree....So we placed funds in a variety of local banks in our service area, we're in what we consider our service area [redacted] county and some of our surrounding counties. And, you know, we're looking for those best rates, we also work through like an [financial advisory firm] for some of those other kind of commercial paper opportunities. And so then we have operating accounts in the local bank. But we need a lot of the services, the more sophisticated services that like a [national bank] would have some of the larger institutions. So in terms of various kind of commercial banking kinds of services. We need those larger facility, more sophisticated institutions. (Female Participant, aged 52)

Experientially, SMEs liking their financiers exhibited paradoxical elements of loyalty and vulnerability for financial enterprises involved with SMEs, regardless of their physical location or scale. On one hand, maintenance of a productive relationship (bank earns money, SME gets what they need) seemed 
to require a low level of continued energy investment on the part of the institution. On the other end, however, there was a supposition of trust in care and protection of SMEs livelihood interests. This is an important consideration in both routine business dealings and at times when increased services may be required, such as during the COVID19 pandemic navigations. Many discussions with SMEs about the nature of their financier relationships suggested a trustbased relationship with a key person. Sometimes this was a bank president, vice president, or other high-level person, who at some point in time had "stuck with them" during both good and bad times. The findings suggest a departure in this type of experience when dealing with a larger or national bank in comparison to experiences with community banks.

It typically ends up and now that to kind of works its way up the ladder. So, okay, I've got, of course, the local banking relationships where I'm talking directly with an owner of a bank and or their, their bank president....Yeah. So I have that relationship. I also have some that have, you know, maybe more than that. Okay. Okay. So I do have a relationship, and l'd have the relationship with someone who's a decision maker. Okay. So that I can feel like when I have a plan, when I have a good property, they've had experience with me after 22 years... (Male Participant, aged 45)

Largely, trust was observed across the findings. However, compared to "likeability", multiple interviewees expressed a more muted degree of "trust in care" on the part of the financial institutions and their operational practices. Expressed as shortcomings in communication proactiveness and limited knowledge sharing on matters of importance to the SMEs' livelihoods, there was sometimes concern about their financiers' interest and capacity to meet their business needs. For the majority of participants, there were levels of trust, yet mixed signaling as to what extent their financiers were sufficiently 
knowledgeable, cared enough about, or prioritizing their businesses' needs. The COVID19 pandemic did seem to raise the awareness of SMEs on who could and would be able to support their financial service needs in times of heightened risk. This was also an essential attribute of value placement on long-term loyalties. Having a financier that had stuck with them in bad times was of exceptional value.

So from my experience, it's basically twofold. They are, they are you can get to the decision maker, okay. And they lend not only on asset value, and necessarily the financials, but there are actually some character lenders still out there. So if you have a deal, it's a little bit outside the box or something doesn't necessarily make sense on paper. They're, they're willing to help. The other thing is, is the quickness in which I can get responses to relatively large decisions. That helps now because we've grown and are in a good position, but these banks were with me, and when, you know, I didn't have any money. And when I probably have less than not having any money, I was probably, you know, close to circling the drain. So I could go to any bank in town. Now I could go to large banks, I can do any of those things. I could probably shave some interest rate off, not a lot, maybe a quarter, maybe an eighth, maybe a quarter. I'm not interested in that. Because in the long term, community banks are the ones that you have relationships with and some of those larger institutions my experience was early on, is that if it doesn't work exactly the way they want it to at exactly the right moment, you might be exiting their grace very quickly. (Male Participant, aged 49)

Contrary to the over-arching theme of widespread trust, a couple

interviewees expressed sentiments of distrust, describing recent experiences as

being "traumatic", "unethical", or wary of bait and switch types of experiences.

I was actually, what's the proper word wined and dined by a few of the bigger banks, courted, I guess. Love the love the brands love the idea saw the vision, I feel. But then at the end of the day, they It was a bait and switch of a not a traditional loan, it was more of a, hey, we have a private equity, we'll buy some of the percent and then sell it back to you piece by piece as alone, which of course, you know, was offensive, that that wasn't transparent from the beginning. Or secondly, that No, and I was very clear with them that I wasn't giving any ownership. I mean, I've had VCs approach me, the last thing I was going to do is go with a bank who wasn't 
necessarily structured poorly, but I didn't want to have to, you know, ask permission from a board sitting down in Texas to for my next move up here. (Male Participant, aged 42)

Another participant expressed a traumatic experience as her business operation was greatly affected by a large bank's decision to close their account when the pandemic hit.

We used [national bank] for 24 years 25 years now. Everything with them was good. We never took out any major loans with them as far as the business but you know, a good day to day banking relationship and so forth. When COVID19 hit they chose to close our account with no knowledge no warning, just our employees went to cash their paychecks at the beginning of COVID and they all bounced and refused to do that when we you know, it took us hours and days to contact someone to find out what the reason was, you know, it was not lack of money wasn't anything like that. And they said they had the right to close any account at any point in time period. (Female Participant, aged 54)

This, however, is an extreme case example from the collected data that emphasizes the range of experiences, particularly during the pandemic. And notably, this instance occurred with a national bank relationship rather than a community level bank, however interestingly, one of the reasons the national bank had been used for so long was because it had a physical location in the neighborhood that was easy access for both the SME leader and their employees. This raises an interesting thought point on trust and value formation related to factors such as physical proximity and accessibility. Nevertheless, for most of the interviewees, any distrustful aspects of the relationship were more specific to personnel capacity for knowledge and fractures in mutual benefit perceptions (from SME perspectives).

Finding 1 (part 2) - Racial and gendered tones - similarities, differences and community "habits." There was no observable difference in 
the trust-trauma range of experiences as indicated by SMEs located in rural or urban areas. Similarly, no obvious differences on the foundational nature of relationships between SMEs with non-white or female-owned businesses, as compared to white and male-owned SMEs, were noted in the transcribed data. Persons of color and women involved in the study expressed similar levels of financier "likability" and "trust in care" perceptions. There were no substantial differences indicated in treatment or perceived access to emergency funding relief programs such as PPP. The lack of such indications was a promising finding, especially since the PPP and Economic Injury Disaster Loan data available as of February 2021 points to under-servicing and lesser access for COVID19 financial relief programs by persons of color, as compared to the white business owners (theinstitutenc.org, 2021).

Nevertheless, it is important to mention that during more advanced and continuous reflections and a revisiting of the notes taken during interviews, there were a couple observable differences in perceived levels of openness and comfort noted during the process. In a majority of interviews with either persons of color or female SME leaders, there was a much longer period of conversation between the researcher and the interviewee after the recording device was turned off, at which time the openness and flow of the conversation improved and descriptive thickness increased concerning how the interviewees felt about their financier interactions and service levels. Expressive thoughts on racialized and gendered dynamics within communities, cultural perceptions pertaining to race and gender and finance, and an increased willingness to use emotional language 
(angry, scared, etc.) became organically active once the recording stopped. Furthermore, in discussions with a few interviewees who self-identified as persons of color, there was some interesting dialogue on cultural dynamics involved with engagements and individualized positioning relative to shared identities, both within the Black community as well as with others. Female SME leaders and owners (both white and non-white) were also observably more cautious with their words and took more time within the interview to seem comfortable with the discussion, in general.

My personal reflections on what these observations provide mostly led to more questions and general concern. Thus, I decided it was important to share this detail, minimally, as part of the findings and suggest continued and more specific research on financial service and knowledge dynamics from gendered and racialized perspectives, including the potential influences of recording vs. non-recording (via technology) within specific population groups (This may be greatly informed by the literatures on gender, surveillance, power, etc.).

As a summary point, the aforementioned observations highlight the value of the study's guiding conceptual framework and its applied pragmatism. By bringing attention to the habitual orientations that may be linkable to interactions (and thus interactional capacity) within a community landscape of interactions, a lens emerges from which to unpack experiential meaning, meaningfulness, and new/change habit formations in fields of such systemic interactions. In pursuit of more equitable finance, which is directly linkable to SDG1 - Eliminate Poverty, perhaps such focus can offer beneficial light for continued progress against the 
ugly shadows of Greenwood, Pigfort, redlining, and other unacceptable scenarios of historical malfeasance in American finance practices. Arguably, such qualitative insights, and more importantly, how they may be useful for fostering inclusive interactional capacity across the human-environment system as a central theme for financial service standard operating procedures, can greatly assist with the addressment of systemic issues of financial inequity and manmade environmental hazards, even preventing some scenarios from arising in the future.

Finding 2 - "Knowing the customer" and "understanding them" is paramount for SMEs. Access, proactiveness, and perceived neutrality (ethics/fairness) really matter too. Banker 'knowledge' beyond established processes is less of a factor. The vast majority of study participants clearly and repeatedly communicated the high priority placement of value "knowing" and "understanding" the needs of their businesses, with a mixed result of how well their current financiers and respective financial enterprises actually did so. There was not a strong correlation between how much SME leaders felt their financiers knew and whether or not the financier fully understood their needs. Also, the size of the financier mattered less than the essence of the relationship, proximity, localness, perceptions of fair dealings, and an assumed community-level identity for the banks the interviewees relied on for services in both normal and crises periods.

I definitely felt that, you know, just to just to use the COVID example, and the support that they were going to be able to give to any other local business in town, because our community is so small, and I don't know, tight knit is the word at least as to what we're supposed to be. But I saw 
there was an expectation. No, it wasn't, it wasn't saying anything bad. I just mean, that's that, that is what we're supposed to be. So there was an expectation that we would, you know, we would receive in some sort of help if they offered it any other small business or any other any of the other small businesses in town. Regardless of how long we'd been open, so there was the expectation that they more or less be there for us as much as they would for any other local business. (Male Participant, aged 45)

Interestingly, specialized financial services providers were reported as similarly valued and capable on these points as compared to local community banks. Such specialized financiers described in the study included agricultural finance, equipment finance, and fintech providers who demonstrated (from the perspective of the SMEs) high concentrations of communication activity on specific industrial topics and financial products tailored to the industry knowledge and needs of the respective SME profile. Hence, even when not local to a specific, place-based community, specialized financiers were perceived as being "close to" the SMEs. Notably, both equipment focused lenders and agricultural financiers were regarded as having high levels of understanding about the SMEs needs, particularly the "why" behind the purchase or loan because they use the product too. In some instances, this was expressed as part of a deep interpersonal connection.

Well, the best thing what I found about them, the two guys I've dealt with are far more easy. In fact, the guy I'm dealing with right now, he farms on the side to me loves what he does. He knows he has to do. So he goes home at night. and on weekends and farms. Yeah. So he understands what I'm talking about. Sure. And he's in the same geographic area. So if we're drafting that, hey, we're sure here are you know, we can talk about the same thing. Yeah. And he's what I love about somebody like him, is you know, he'd rather meet you on the farm or he'd really come down here and talking to tuition, right? I'll come ride your tractor with you. It's a personal thing. (Male Participant, aged 65) 
Interestingly, in these scenarios there was most often an awareness that the pricing (interest rates, fees) may have been slightly higher, yet still deemed as competitive and in line what is presumed to be a fair exchange for the ease of the transaction and embedded knowledge about the product(s) - financial or otherwise (e.g., short term loan, integrated reporting, equipment, inputs, etc.). This suggests a few scenarios: 1) SMEs may perceive some profiles of financiers, whether community-level or larger in scale, do not fully understand the level of knowledge and associated agency on the part of SME in a given transaction, 2) financiers seem to work to cultivate (and may have the power to do so) a specialness or stickiness to the relationship, and 3) financiers and SMEs apply a premium value on what they have in terms of the relationship. Or simply put, exactly why the SME chooses to do business (often repeatedly) with a financier can take many forms. This is interesting and non-problematic on face value; however, it does raise the question of origination principles of such premiums for financial service and the importance of structural fairness (Hendrickson et al., 2018). Furthermore, it points to vulnerability for financiers who do not maintain what is perceived to be enough value for the SME business leader. Lastly, moral hazards can erupt if equitable governance principles are not carefully guarded.

In discussions with the financiers who were interviewed, it was made repeatedly clear that there is sustained interest in understanding as much about the SMEs (both the company and their leaders) as possible, as well as being both accessible and embedded within the communities (place-based or virtual 
and specialized interest) they serve. This commitment is real (socially), of strategic importance to community financiers, and observable as very action oriented. Notably, large and small financiers were both described by SMEs as relationship oriented but executed their strategies for client acquisition and care somewhat differently. For both, making (and keeping) some type of connection was paramount. However, the local, community banks utilized more personal and direct social networks for increased business, whereas the larger banks (and more distant actors) worked from both the personal connection as well as aggressive market share acquisition techniques.

So we focus primarily on the neighborhoods we serve within (redacted) as the greater community. And so [redacted] would be a smaller neighborhood within [redacted] can centrally located over by, you know, in the [redacted] area of [redacted], and then [redacted] would be our second primary neighborhood in [redacted] as well. And so we focus our core relationship, our communities that we serve in that area, and then it spreads out to [redacted] and a little bit into [redacted] and [redacted] as well from there. So we primarily focus on those, we don't actively reach out for business. Business kind of comes in on a referral basis as well as through our president, his relationships as well. So we're not actively cold calling folks or anything like that. Business comes into the bank by customers referring as well as employees, referring them from their personal professional lives, as well. (Male Participant, aged 32)

The community bankers and each of the SMEs interviewed indicated "understanding" the client and "knowing" the needs of the SMEs as a priority for successful relationships. Achieving this, for some, was suggested as becoming more of a delicate balance, particularly as paradoxical aspects of financial technologies fostering more data capture on some levels, but also creating distance and lessening the "touch" between SMEs and the financiers as they navigate their relationships. This has real implications for soft information 
exchanges, "knowing" the person, or having them "look you in the eye"; all factors that have historically been of significant influence in relationship lending and community finance processes.

\section{Q2. How do community-level financiers engage with SMEs and does ‘community finance' and/or 'sustainable finance' knowledge exchange occur?}

In the course of normal business, financiers engage with SME leaders/owners in numerous ways, many of which can be labeled as "expected", including face to face (e.g., on premise, formally or casually), email, phone, letter correspondence, social media, apps, website portals, and community signage and periodicals. SME leaders/owners communicate with their financiers in the same ways as listed above. Approaches to information quality and exchange dynamics were observed as what was customary for the relationships and general preference of the SMEs. In general, all study participants indicated deep and frequent levels of information exchange are welcomed in both "normal" and "crisis" periods. The latter frame was described as necessitating more timeliness, depth of subject matter intelligence on the part of financiers, desired proactivity in program communications, and "care". Neither community finance nor sustainable finance were found to be explicitly discussed or overtly promoted by financiers to SMEs. However, community finance was repeatedly conveyed as real and present by interviewees as part of the interactive engagements they have with their financiers. Furthermore, community finance as an experience was 
perceived in both direct, and more typically described indirect communications and patterns.

I think those that do want to invest in their communities, I would say probably do better. Okay. And I bet their deposits are higher. Okay. And those relationships are probably a wider net has been cast. I think those are all positive things. But yeah, that's kind of what I've experienced is with larger banks, and l've dealt with global banks, okay. It's just the lack of personal touch is evident. And when working with a community bank, it's just I know everybody's first name, I go and walk and walk in there and say hi to every single person that's sitting at every desk and that's just not something I can do at my other banks that I've worked with personally, right, you know, a larger I don't know, I don't know. A single person, Okay, first, like a [national bank], I don't I couldn't walk into a branch there. I couldn't tell you a single person that works. Okay. That's just a difference. It's just way different. (Male Participant, aged 40)

Community banks' and specialized financiers' frequency levels and standard operating procedures for client communications were generally regarded as positive, including during the COVID19 pandemic navigation period. Both SMEs and financiers reported satisfactory frequency of information exchange. Financiers who were interviewed generally portrayed themselves and their institutions as doing as much as possible to provide relevant, quality information to their SME clients at all times, even getting creative when necessary to meet the needs and expectations of clients. A willingness to constantly work to do more was also extracted from these conversations.

Yeah, whenever you're just in a one building branch becomes challenging, but you limit the traffic, the foot traffic inside now, and we did a lot more drive up or, you know, window, consultation or discussion. To go a step farther, you know, we're outside, we're saying that we're okay. Or at least it's a little better. So we have done more talks on the sidewalk in front of the bank or are closing loans on the hood of pickups inside, go to the drive thru and closed loans in the round. I mean, so when there's a challenge, we try to mitigate, and still continue to serve the way that people have come to expect us to serve them. As well as meet the needs of the future customer. Through advances in technology, actually so we now have 
more branches if you want to classify a video ATM, which is an interactive video teller machine. Yep. So it's like an ATM but you also get the capability or have the capability to visit with a live person, okay? You can conduct transactions, just like you were standing at the teller or at the drive-up window. (Male Participant, aged 42)

\section{Finding 3 - Information exchange dynamics are attributed to the}

human actors, not the enterprise/financial institution or its characteristics.

The topic of information asymmetry is prevalent in the relationship banking literature. Its occurrence was also observable in the findings of this project. Several SME leaders indicated opportunities for improvement on the part of financiers sharing information in ways that are equitable supportive of all customer interests, and proactively expansive (e.g., new knowledge about a product that would be good for the SME business). To clarify, the SMEs did not express frustration or concern about information asymmetry, but simply that more balanced and inclusive information exchange models could be achieved, and they would be appreciated. Participants expressed "strength of bond", trust, and temporality of the SME and financier relationship as factors contributing to knowledge and information exchange between themselves and their long-term finance partners. From the SME leader perspective, the attitudinal approach to information exchange activity level was somewhat varied, with participants acknowledging the importance of giving the bankers what they need, but purposefully not much more than was requested. Multiple SME leaders also conveyed feelings of annoyance when it was perceived as if too much information was being requested.

They want to drill down as far as your customer base as far as your larger accounts. They're asking more questions. Okay. Our bank that we use 
also finance as part of a finance group for one of our, our second largest customers, so they have a relationship with that. And unfortunately, that customer is going through some things due to COVID and the (redacted), which is $80 \%$ of their business. So the bank has been very I almost say intrusive, asking too many questions. Especially with a new point of contact, I understand that he is trying to get a grip on what we do, but he's asking these non-industrial questions. So he really has no idea what our business is or our model, it's pretty simple [redacted]....you sell it, you mark it up...or you sell it by marking up whatever making a profit. And it's almost to the point that it's too intrusive, that's annoying and kind of pisses me off, to be honest with you. (Male Participant, 48)

Notably, more balanced information exchange (both hard and soft) was attributed to SME relationships with smaller community banks, largely due to the interpersonal connections. SMEs involved with larger regional and/or national banks raised some level of concern about inadequate, one-sided information flows but not to the extent it was prompting considerations of disrupting the relationship. In terms of information quality, there were mixed levels of concern about the quality of information from the varied sizes of banks discussed in the study. In general, the size or profile (brick and mortar, online only, specialty) of the financial institution was not the priority characteristic identified by study participants as a main consideration pertaining to information access or quality. Relationships with the larger firms - national banks, fintech, and others - are not as characterizable by active dialogues and balanced information exchanges in the same way as local, community-level relationships. This was a distinction between experiences with large and small financiers, however the impact of information (a)symmetry was not observed as being a clear or fundamental requirement for successful SME and financier relationships. 
Furthermore, some SMEs working with both large and small bank financiers' shared experiences of infrequent experiences of disinterest, inaccessibility, fuzziness (lack of clarity), missing contact person, confusion, and organizational shortcomings on product and risk navigational knowledge. These instances occurred at both large and small community financiers, and garnered some frustration, yet on the other hand, there were several indications of imbalanced information models that were completely satisfactory to the SME, regardless of financier size, locale, and form. Overall, the findings were generally mixed to positive on satisfaction with information exchange habits, with transactional knowledge exchange regarded part of status quo and soft information indirectly expressed as being more valuable. Any perceived shortcomings or imbalances were not experienced as overtly intentional, with the exception of bankers asking "too much", but rather as a function of personalities, government policies, or personnel capacity limitations. In general, open communication patterns and free decisions to share (or not) around both hard and soft information were the most valued characteristics of what SMEs considered relevant for productive information exchanges that yield desired financial transactions.

But he wasn't obligated to give me a loan, in fact, had I not sat down with him, I never would have got that loan. But in that sense, he and I build a relationship there, whether it's spoken or unspoken, that he got faith in my ability to pay for that loan. And once he had faith in my ability to pay that loan, then he made that loan. That was the only obligation here. To me, that's the only obligation I think a bank has, that if somebody is worthy of a loan and wants a loan, I think that you should provide them some sort of a loan. Okay. I mean, obviously, there's competitive marketplace where some rates are higher than others are better than others. (Male Participant, aged 41) 
Important observation-only themes related to information symmetry, or perhaps what should be more accurately described as information exposure/awareness, were also present in the data. These included observed differences in gendered confidence, individual agency, and levels of activeness regarding perceptions of financier choice-making, relationship dependency, and expectations of information quality.

It was observed that some female led SMEs were possibly more accepting of imbalanced information dynamics as compared to male interviewees who participated in the study. The males described relationship scenarios of with information exchange habits perceived as symmetrical and expressed confidence when it was determined that if action needed to be taken to engage the right financial partner, then the information to do so was available. It is important to clarify, female SME owners did not express perceptions or habits associated with victimhood but were simply less bold in expressing confidence in ability to make a financial partner change, and in some cases, distrustful about what may come if with a new financier relationship. For smaller scale firms, there was a real hesitance to consider making a relationship change even when perceptions of underservice were present. Females involved with larger SMEs did not exhibit similar characteristics, but notably some were also not the owners of the SME which may have been a differentiating factor. This divergence of demonstration was limited to gendered comparisons and was not found to be evident between white and non-white males involved in the study. 
The temporality of the SME/financier relationship correlated with perspectives of trust and mutually beneficial information exchange. Nearly every SME leader that indicated perceptions of symmetrical, trusting information exchange models with their bankers reported characteristics of 1) lengthy relationship, and 2) a period within the relationship when the financier "stick with", "bet on", "took a chance", or "worked with" the SME when there was a situation of enterprise distress, ranging from recent start-ups/expansions to decades old businesses. Descriptions of the modality of information exchanges included bidirectional openness in dialogues, genuine interest in the business and household well-being, very active questions on the part of the financier(s) and the SME, and trust.

I've had this discussion with Mr. [redacted] several times, quite frankly. And he's, he goes, I mean, he, you know, he would, he tells me whether they're good shape or not good shape, or well, you know, they only have so many bad loans and where they're at and stuff. So I feel comfortable. I know, we're not insured. And we can play around with some ways to do that, but I feel comfortable...From a community perspective, I think it is, though, a success for people where there's a relationship where they know your business model and your business model involves financing. And that works. Because you know, it's revolving accounts or a refinance, or those types of things. (Male Participant, aged 71)

The quality of information that was exchanged and depth of perceived knowledge was not as celebrated, compared to experiences of open exchange across the collection of interviews. This was most reflective in the dialogues pertaining to the content and processes of information exchange related to crisis period navigations. For some interviewees, when information exchange was perceived even to be balanced there were some concerns about the quality and intended use of information that was moving between the SME and financier. 
The primary driver of such concern was associated with perceptions of lower levels of knowledge on the subject matter of interest (what was needed in the crisis response) and to a lesser extent lack of effort between the actors.

So I started the business doing kind of signing up for my bank account and my point of sales all in one. So the bank I am with is [global bank]. And the processing for my retail business is [merchant processor]. So [merchant processor] has the software and they sell the hardware. So I purchased a [merchant processor] station which allows me to take credit card payments and iPhone payments. It flows the payments into my [global bank] account the following day. So it's very nice. The rates are probably a little bit higher than more national products a little bit sense of ease and since I am in the [redacted] industry, my business is [redacted]. So I just wanted to kind of keep my head down. Just get it open. If I'm going to get a bank account, and they're not going to ask any questions. I'm just going to sign and get it done. All right. I don't have any credit available through [global bank], so they won't finance, my inventory. (Male Participant, aged 47)

\section{Finding 4 - 'Local' finance landscapes: SME and financier}

\section{relationships as an aspect of community finance are paradoxically}

complex. In the study, the landscapes of financial interactions for the interviewed

SMEs were somewhat varied, ranging from $100 \%$ local to virtual-only

relationships, but the more local or place-based relationships supported the type of soft information exchange that is prevalent in the literature as somewhat of a "secret sauce" to what makes community finance so resilient. At present, community financiers remain mostly place-based and embedded within local, physical community, or regions. This landscape of interaction offers multiple opportunities for information exchanges between these actors, in both historic contexts as observed and in future-oriented conceptualizations. Such interactive exchanges hold a variety of characteristics including formal/informal, intentional/unintentional, verbal/nonverbal attributes. Both SMEs and financiers 
indicated a general acceptance of such modalities in the nature of productive relationships. From the community banker perspective, intentionality of fostering deep and sustained interpersonal connections was prevalent. For a majority of the SMEs interviewees involved with small to regional sized community banks, both local access and community as a place was perceived as beneficial to the interactional capacity and overall characteristics of physical and cultural landscape. These elements offered opportunities for knowing one another and experiencing interactions directly, both formally and informally, and through broader community interaction.

We try to do two things. One, try to put the customer's needs first. So it with timely decisions and processing. So that way, they're not wondering where we stand or what it is that we're thinking, okay. It's an open dialogue. Yep. And then we have more people come to us and ask for general advice. Whether it be about personal finance in their homes, or whether it be how to structure their business, or how to acquire and make acquisition. I mean, so I feel like we have really set ourselves apart, not just the customer service, the customer service, I believe is always there. Doing it. Yeah, that's a whole other thing. This is more relationship building. Okay. And we have been doing that, without even knowing we were really doing it for a long, long time... Yeah. So l've talked to some, some business, several business owners and l've talked to some other banks too. And, and the, the topic of character lending, which is kind of what I think you're describing here has come up repeatedly. Like it's been, so it sounds like and I don't mean character, like oh, that's a good character or that's a bad character but getting to know the person and the bankers at multiple levels. Doing that here and talking with the business owners in different ways and whatever they want to achieve. And you would say, that's very much so the way this works, too...Well, I think so. I mean, you, you initially have a certain level of comfort. Yeah. From a lending perspective or a business relationship perspective. Yeah. But if that's going to grow, yeah. And in the end the business customer wants to grow. Yeah, they obviously can't be just surface level, information givers. Sharers. We have to dig, we have to be able to trust each other on a deeper level, especially when you're talking about some of these businesses moving into the millions of dollars of operation. Yeah. You don't just give someone a million dollars because they want it. (Male Participant, aged 42) 
In general, the findings overwhelmingly suggest community banks want to bank within their communities. Furthermore, SMEs and bankers both expressed the value of the other and perceived themselves as having a good understanding of the others' operations and needs. However, as regulated enterprises, this is sometimes not as easy as a simple, local decision on the part of the banker(s). Information, including behaviorisms, habits, multi-generational resources all may contribute to what transpires between an SME and financier, with both positive and negative outcomes. In brief, too much information was described as both good and bad by both SMEs and financiers engaged in local, community finance interactions.

Interestingly, there was also a finding that the interactional attributes of SME/financier relationships with place-based community banks in localized settings supported interactions and afforded opportunities for businesses (and their owners) that may not have transpired if certain soft information was not readily visible for the financiers. Upon initial reflection, this seems to be problematic in a variety of ways, a detail which should not be minimized. "Nepocronyism" is a creative non-word term this research study uses to visualize the simultaneous presence of kinship and compounded social relationship dynamics to express just how intertwined SMEs and community banks may be in hyperlocal landscapes, such as rural settings or specific neighborhoods. This logically can present both very real problems, yet at the same time very creative and beneficial solutions, as the interwoven aspects of daily community life for SMEs and their community bankers have many touchpoints. For example, if you know a 
lot about someone or their family, there may be greater confidence and informal documentation to proceed with a character lending transaction. This can be a very positive and good outcome at both the individual and community level. However, the possible problems include exclusionary practices of nepotism and cronyism, preferential treatments associated with multi-generational families, and the inadvertent propagation of systemic discrimination (e.g., racism, sexism, ageism). Repeated reflections on this observation re-emphasize the importance of safeguards, policies, and leadership training to protect from such outcomes in order to foster more inclusive social-ecological principles within the financial organizations. It also brings forward a call for continued and deeper investigation into these paradoxical aspects of community banking. How much and what types of information, particularly the soft information, is enough, is too much, or is not enough? And importantly, is quality information and transactional capacity being equitably distributed? In addition, these complexities also prompted ideation around the role of technology in supporting more flexible financial service frameworks that allow for any positive characteristics of "nepo-cronyism" to be deployed in inclusive and fair formats via more open systems for communications, financial decision modeling, and dynamic risk assessments, as compared to what is operationalized today.

Finding 5 - Community banks were the lifeboats to many SMEs navigating COVID19 stressors, yet the most trusted source for SME navigational knowledge in both "normal" and "crisis" periods may be their accountants. Varying levels of confidence in financier knowledge about SME 
business operations, financing product needs, or programs that may be of importance to SMEs particularly during the COVID19 pandemic, were noted throughout the data. Community banks were the favored actors in SME financier relationships when a perceived co-navigation scenario was unfolding. Some exceptions existed, particularly with agriculturalists and health and wellness businesses who indicated closely working with specialized finance entities they thought really understood their business needs and took care of everything. In some instances, this group of interviewees also worked very closely with community banks but largely for different reasons beyond ongoing navigational knowledge whether in routine business dealings or periods of crisis response.

In terms of COVID19 responsiveness, both community banks and specialized finance entities were indicated as having performed as well as possible in meeting the SMEs' navigational knowledge needs about the COVID relief programming, with no sustained negative implications arising via the colearned process.

It went very smooth. It did, okay, a banker called me said, hey, they're doing a PPP. When can you come in? Okay, l've been doing my homework on it, this is what you need to do get your account with me blah, blah, okay, did a couple of days work due to bugs just to let you know, boom, okay, then the other bank that I did on the other side and other agency, one was on the [redacted] side and one was on the, on the fair, two different entities, right. And so the other bank, when I do most of my [redacted], they call I met with them, they got the application, the guy wasn't as knowledgeable as much smaller local bank guy was, but because we already did on that side, I knew what needs to be signed in. Okay. Because I work with my senior vice president we met and got together on the other relationship did I didn't that's a bigger bank. Thanks. So, I have a good relationship with the guy but he was younger and doing ....he seemed he's involved in a lot of levels with this. And so I was able to get that paperwork filled out pretty quick. (Male Participant, 57) 
In response to questions about navigating COVID19 related knowledge, the majority of SMEs reported working closely with their community banks and specialized finance entities to access relief programming, primarily PPP loans. Overall, these encounters were described as positive, even as they were perceived as including ever-changing rules that were indicated as confusing for both the SME and the financiers. For those SMEs that had relationships with both community and larger, national banks, there was a heavy reliance on the community banks and specialized finance entities, as compared to the national banks. The rationale for doing so included expressions of perceived ease, accessibility, and care. For those working with both community and large national banks, the range of experience was notable, but similar to most of the experiences with community banks, if and when there was access to information, a trust-based relationship, and a perception of care, the experience was conveyed as generally positive.

And [national bank] did very well by him to get his you know, he gave him all the details he showed me what he was gonna get...But yeah, I think a pretty good experience. (Male Participant, 59)

The observable impact of the right combination of information quality and quantity and trust has toward the development of perceptions of care was also recorded in discussions with an SME using a fintech firm as their primary financier and to navigate COVID19 program access.

I've been with [fintech] for eight years and I've never had a problem with it. I am unbelievably impressed with that company. Okay, they are. They had an worldwide shut down like blackout of all their stuff l'd never been communicated so well with here's what's going on. Here's how we're getting everything online. Here's how you're going to take the money here. Yeah, this and that. So I have never left them even though people have 
been like [fintech]. I'm like, no. Awesome. Okay. I've also taken out their [fintech] capital, okay, which you can and what l've liked about it, how do you know about these programs with [fintech], they tell you about them, they shoot them out to you in what? In what format so you can go look on your dashboard. Okay, it is all there. There's messaging on the dashboard all the time there. (Female Participant, 50)

However, when expediency or nimbleness was not perceived as possible due to institution size, or shortcomings in information quality and access were expressed, a notable shift in awareness of smaller, community bank value was present.

Well, I think the problem with big banks, being involved in certain community activities is the world's moving fast now, and things happen fast. The banks big banks have is they're, they're clunky. And it takes a long time to move the ship. And so like, the PPP is a prime example. It's the case study, they had no ability are huge, or whatever. And it's certainly a sincere and fundamental gesture to try to, to try to move again, I don't want to be cynical. I think there are some organizations that are doing things for the right reasons, and do have very civic minded folks. But as a rule, I just think it's more difficult to organize people and like, for example, if you've got, you know, some natural disaster or what have you, I mean, short of giving money, I think it's just really hard for them to get all their department heads together and get everything organized in a timely manner. It's just seems like smaller organizations have the ability to be more nimble. (Male Participant, 59)

Regardless of who or how the crisis navigation discussions between financiers and SMEs were initiated, it was clearly articulated in nearly every interview that the SME's financier was an active and trusted partner in conavigational management of the COVID19 crisis response yet was not identified as their most relied upon resource for higher level knowledge for business management during both normal and crisis periods.

Interestingly, the SME network relationships that were consistently identified as most knowledgeable and proactively informative were third party 
accountants. Simply put, as SMEs navigated the COVID19 pandemic's impacts on their businesses, they asked their accountants what they should do, and most, if not all of the accountants knew and offered up guidance and solutions. SME activeness regarding critical decisioning around PPP and EIDL, as well as available county and city government relief programming was typically in response to directives received from their accountants. Unquestionably, the interviewed SMEs perceive a level of dependence on positive relationships and information exchanges with their bankers; however, the most valued player status concerning knowledge and information in periods of critical, crisis-oriented decisioning seems to belong to SME accountants. Nearly every SME in the study reported following the guidance of their accountant to operationalize their response in conjunction with their bankers. Observably, this seemed to be an extension for how they also approached deeper knowledge and compliance requirements in the course of normal business operations.

I mean, if anything, l'd say my CPA would be more my business bank just because, you know, I pay my taxes quarterly. And then, you know, he or whoever it is at the firm, you know, they do my taxes for my business and take care of everything. (Female Participant, aged 46)

\section{Q3 \& Q4: Are narratives of 'community finance' and 'sustainable finance' related? What is the experiential essence of each? Are they related in terms of experience - in both 'normal' and 'crisis' operational frames?}

Community finance and sustainable finance are not solely grounded in economic factors, regardless of landscape (virtual, physical, etc.) placement, scale, emphasis, or interactional capacity. This holds true for the broader orientations of finance and its embeddedness within nearly every global facet of 
modern society. Structurally, banks are social constructs that have been central to the human experience throughout much of history, affecting vast outcomes in the natural environment and socio-cultural landscapes at large. Experientially, finance is a principal component of daily life for businesses, households, and institutions ranging from governance to market orientations to language, to name a few. Finance is both powerful and facilitative in past, present and future contexts of the human-environment system and related interactions with each other and the biophysical environment. In brief, exploring its realms offers us a perspective from where to unpack what we (humans) are in thought, action, belief, and habit with sustained social-ecological effects.

"If the financial system has a defect, it is that it reflects and magnifies what we human beings are like" (Ferguson, 2008, p. 13).

The findings below, which are associated with research questions three and four, have been combined in their presentation because there seems to be no benefit to presenting them as separate. In our modern, technologically enabled society, the reach of information, as well as the formations of communities are no longer limited by proximate geographies. Information is everywhere, people are connected, linked, and engaging, everywhere. Thus, narratives of sustainable finance and community finance are arguably part of the experience, everywhere, even unknowingly, or minimally when considered from the perspective of potential exposure. Nevertheless, the experiential essence and related consciousness of community finance and/or sustainable finance has not fully engaged everyone or yet been made possible everywhere by techno-society's 
progress, thus far. So there remains a distinction between the narrative and the experience. Consider the SDG framework and sustainable finance agendas as important and relevant examples, whereas the narrative is quite active and what is occurring in practice is still limited and/or constrained in many ways. For this reason, considering what community finance represents, if anything, to sustainable finance, presents an opportunity to unpack the relationship between narrative and meaning.

Finding 6 - COVID may have unveiled the 'value(s)' of community banking - co-experiences, collective resilience, and crisis/hazard management. (NOT non-emergency risk management). In alignment with the broader data sets on COVID19 and its financial implications for small-medium businesses, the sample population's experience with community financiers as part of their businesses' COVID19 navigational response, was overwhelmingly positive. For the interviewees who participated in PPP funding, there was a generally positive dynamic with the overall process, even as situations of limited or incomplete knowledge and process confusion were described. Similar to how it was observed that community banks want to bank their communities, it was observed that community banks genuinely want to help their community-based SMEs, particularly in times of hazard/crisis management.

Ease of access, localness, and availability of human interactional capacity on behalf of the bank financial enterprise were perceived as contributing factors to the responsiveness of community banks successfully assisting with PPP. The interactional capacity of small, community banks is what significantly 
outperformed, at least experientially, the larger financial institutions. Bank size was marginally perceived as a factor in how much support SMEs received from their financial partners, with exceptions occurring for both fintech and specialized sector organizations (agriculture) when it was perceived that someone was taking care of the SME's needs. The important point centers on the necessitation and value placement on interactional capacity in time sensitive situations, Interestingly, this finding is specific to real-time risk and hazard management as opposed to longer-term strategic management of SME risk. In the latter, themes of searching for superior knowledge of SME needs, more robust product access, and scale of resources were increasingly involved in financial risk management decisions that affected financier selection and engagement processes.

In addition, SMEs seemingly appreciated a shared consciousness with their community bankers as being "in this together" and expressed high levels of patience and value in co-navigation experiences. It appears as long as the information exchange was open, and the community finance field was perceived as inclusive (enough) for the SME's needs, the interactions with the community banks were perceived as positive, productive, and greatly appreciated.

Finding 7 - 'Sustainability' as part of finance (community finance and other) is complex - profitability and care of funds is expected; community obligation is not.

Discussing sustainability as part of finance yielded mixed findings. When asked about their thoughts, beliefs, and perceptions about the relationship of sustainability and sustainable development (often with post-interview 
explanations of what it "represents") to community finance, as well as the role of financiers to facilitate knowledge or activity associated with environmental and societal sustainability, most SMEs expressed they did not have an expectation of sustainability linked engagement from their financial service providers. This was indicated regardless of bank size or profile they utilized. Approximately half of the interviewees actively communicated no desire for sustainability knowledge or guidance from their finance partners and the other half primarily associated sustainability, the term, as a profitable state for businesses and banks alike, and expressed their bank's (and others) priority related to sustainability as remaining solvent and "care of funds".

Furthermore, there was only a weak association of any type of obligatory role financiers have toward a community. Interestingly, the SMEs interviewed repeatedly communicated the appreciation, activeness, and overall benefit of having financiers within their communities, yet most of them did indicate feeling the role of 'community financiers' was one of free market activity and that community-level contributions are not an expectation. What is being experienced was communicated as different from what is expected, which I find to be quite interesting. There were a few exceptions to this over-arching theme - a couple SMEs in urban settings that hold relationships with both large financial firms, as well as an agriculturalist who was interviewed in the study, both expressed feeling at least some level of obligation on the part of community banks to contribute to communities, particularly if they are overtly labeled and marketing themselves as a community bank. 
Finding 8 - Community finance is "everywhere", sustainable finance is not yet surfacing as part of the relationship experiences of SMEs and community financiers (at least not in the Midwest). Sustainable finance and/or sustainability as a topic were not found to be present in SME and financier interactions, regardless of SME industry (e.g., agriculture, real estate, food service). Community finance did not present as a direct association of sustainable finance, even as community finance was repeatedly perceived as a demonstrated, experiential aspect of SME and financier engagements. Similar to community finance, but to a far lesser extent, there were a couple embedded elements of sustainability (not 'sustainable finance') in modes of information exchanges (e.g., e-statements, conservation planning) and in discussed profitoriented objectives for the SME to be sustainable over the long-term (e.g., care of land for highest yields). These experiences were not described by either SMEs or financiers as directly associated guidance, recommendations, concerns, or transactional requirements involving sustainable finance. Community finance, as an experiential process, was found to potentially have space for the support and facilitative leadership on sustainability projects, with increased linkage to sustainable finance. Yet, at present, sustainable finance was not found to be a familiar concept to either SME leaders or community bankers and SMEs are not asking for it.

Nobody's saying. As an example, I want to finance a giant wind farm. Can you help me? (Female Participant, aged 51)

Interestingly, each of the bankers that was interviewed expressed interest in the topic of sustainable finance even though they were largely unfamiliar with 
the terminology. Also, for both a few SMEs and bankers there was personal knowledge and interest in the SDGs. When asked specifically about knowledge and ideas of sustainable finance there was observable pause, yet active discussions on its follow-up $d$ with potential to serve the interests of both financiers and their SME clients. Furthermore, a couple SMEs indicated being very active in sustainability pursuits due to their own personal values. 


\section{CHAPTER 6 - Discussion and Implications}

In the 1950s and 1960s, Irwin T. Sanders worked to prioritize community as a social structure (as did others before and after him), arguably to advance its centrality into a conceptualization of systemic interaction and social reality formations across society. In his book, The Community, he rather casually stated that his objective was "revealing how ordinary activities in ordinary people fit together in a comprehensible social world we call community” (Sanders, 1966). This social constructivist rationale for what community means is as relevant today as it was 70 years ago. Nevertheless, the simplicity with which he approaches community as "ordinary" interactions is what appeals to this research agenda. The conceptual framework proposed by this study is also not overly complex, but rather works to simply present a reminder to those engaging with sustainable finance that community finance is very real, very ordinary in some regards, and at the same time quite extraordinary and capable of being actively engaged for successful sustainable finance deployments. Also, as SMEs are quite small when looked at singularly, they perhaps are being overlooked in terms of their collective footprint and power to realize SDG progress. In smaller, more rural landscapes, SMEs and community bankers are both quite influential in multiple ways. So is the community finance they do, together.

This study's conceptual framework centers community finance as a process because it highlights what is arguably missing, to date, from many sustainable finance operationalizations, and more broadly the sustainable development goals: the community processes and mechanisms of interactional 
capacity concerning the global agenda. The interactions between people, in daily life, contribute to meaning formations in numerous ways. They generate emotion, they contribute to knowledge gains and losses, they facilitate social constructs of attitudes, and more. This is very true for SMEs and community bankers engaged in community finance as part of connected livelihood pursuits, as well as in their de facto positioning as local leaders. As these actors are comparatively autonomous decision makers, and both socially and economically crossnetworked, they are socially, ecologically, and culturally bonded. Furthermore, they have proven to be resilient in periods of high hazard, such as the COVID19 pandemic and the earlier 2008 financial crisis, as well as before Therefore, they should be collaborators on projects, solutions, and creative ideation for SDG linked mobilization. Arguably, if SMEs and community banks are not actively being engaged in the sustainable finance frameworks, sizable opportunities are being missed and avoidable problems may arise.

How community finance as a process relates to community level meaning on sustainability and sustainable development can shed light on broader societal trends. From the perspective of enhanced real-world applications (faster progress) of the SDG objectives, particularly as characterized by SDG17 partnerships, the missed opportunity can easily be envisioned. Not surprisingly, the growing body of sustainability, ESG/CSR, and sustainable development literature presents repeated calls to action for increased contextual inquiries, as others are noting there are missing elements for fast-enough progress toward the SDG goals (Bogna et al., 2020; Lagoarde-Segot, 2019; Theodori et al., 2016). 
More details regarding the "ordinary" in constantly changing landscapes of community interaction, as it relates to society-level goals of sustainability can provide insight into what is happening, and particularly becomes interesting when considering new ways for engaging social actors that can have an outsize role to play in delivering real results because they intentionally work to do so (King, Felin \& Whetten, 2010). Excitingly, these actors are already engaged in active information exchange processes. The level of SDG awareness is certainly evolving, as calls to action on climate emergency navigations are now reaching new actor groups, seemingly daily. Nevertheless, there is more information available on the SDGs than ever before, yet it seems SMEs and community bankers are not experiencing exposure to relevant content. Since the finance and investment industry, food and agriculture companies, non-profits, and governments everywhere are increasingly working to engage on the topic, then ensuring quality information becomes available for key decision makers in roles of influence in networks across the U.S. landscapes is imperative.

This research presents useful, qualitative insights into regionally active and globally networked actor groups that can actively participate in the sustainable finance agenda, if given the opportunity. SMEs and community bankers, alike, hold positions of outsize influence when compared to other individual profiles (e.g., employees and volunteers) due to their decision-making roles in business operations (such as hiring), increased wealth resources (successful businesses), and community-level investments (charitable giving). Ordinary interactions between financiers and SMEs have provided an informative 
frame for exploring what is exchanged between them in routine interactions. Productive consideration on the opportunities (and risks) related to engaging community-level financiers and SMEs (or not) has not been appropriately actioned in the global sustainable finance narrative and its related operationalizations, to date. From this perspective, the findings of this research and the argument presented by Migliorelli (2021) are supported. As a starting point, sustainable finance should be reframed as "finance for sustainability" (Migliorelli, 2021). Furthermore, this research works to spark a discourse on how this may be conceptually advanced when community as a social structure and community finance fields as processes are embedded in the logic argument. Reframing to better link community processes and relationships dynamics to sustainable finance is imperative for it to "work" and not leave persons behind, either in actuality or perceptively. Through conceptualizations of community finance for sustainable development and sustainable finance for community development, a more obvious linkage between community-level dynamics and sustainable finance can be envisioned.

Importantly, the landscape of SME and financier interactional experience acknowledges both the fluidity and complexity of the lived situation and can be relatable to sustainability knowledge that is gained or loss via interactions. When conceptualizing such activeness, it is not hard to consider how much more may be achievable in response to the SDGs and their critical timelines that are communicated as being urgent. As mentioned in earlier chapters, there is no single definition of sustainability or sustainable finance, yet some forms of their 
meaningfulness (or rejections of) are likely to emerge when exposures occur due to media, commercial interactions, or personal interest navigations. Perceptions and behaviors, as well as attitudes and beliefs are experienced as real on individual, enterprise, and community levels. If positive attitudes and actions related to sustainability (in line with the SDGs) form across a community of interactions, positive results may emerge. Likewise, the opposite can also be true. SMEs and community bank interactions offer a facilitative mechanism for transmitting factors that can and will contribute to either outcome, and if they are not actively engaged on the subject matter, or even develop negative associations with the SDG agenda, this becomes a sharable and contagious development in the wrong direction. Purposeful engagements with community financiers to advance SDG related programming for SMEs offers an interesting model for exploring what is possible in both urban and rural settings across the social-ecological landscape. This may be particularly true as community finance, as a process, is already socio-culturally embedded and highly valued for those engaged with it.

\section{Community Finance as a Process and Its Social Relationship Dynamics are Highly Valued. Sustainable Finance is Missing (So Far).}

In discussions with the $27 \mathrm{SME}$ and community bank leaders, it became evident that community finance is almost intuitively known, broadly accepted, and favorably perceived by SMEs as playing important roles for their businesses' success, as well as the wellbeing of the communities with which they identify. There also seemed to be at least a partial awareness of community banks' 
importance to a well-functioning U.S. financial system. Study participants indicating knowing community banks are important to the financial and community level ecosystems, expressing multi-dimensional aspects of their presence in economic, social, and cultural matters. This was observed as a similar value orientation in both urban and rural settings. Experientially, SMEs truly and deeply appreciate their community banks for what they do in their communities and conveyed a general acceptance that community financiers are not necessarily the low-cost provider for financial products and services. Nevertheless, the value equation is perceived as beneficial for the SME, as an enterprise leader, and to meet the needs of their identified and cared-for community. Interestingly, this finding was similar across both place-based and identity-based (e.g., sectoral) communities. The narrative of community finance was found to be primarily passive in orientation with little direct activity of community level marketing or other overt, nonpersonal communications. When the narrative was noticeable to the SMEs, it was observed as associated with actions such as locally shared interests such as charitable contributions or landscape improvements that benefited the entire community. Seemingly, community finance narrative may inform early perceptions about what a community bank does or can offer, but the activity landscape, what is actually done and experienced via interactions, is paramount to its essence.

Sustainable finance, in neither narrative nor action, was found to be experientially present during the study. Most of the interviewees had not heard the phrase "sustainable finance" or had only a non-work related awareness of 
what the Sustainable Development Goals represent, thinking of it, moreover, as related to individual or household decisions such as recycling. Both SMEs and community bankers expressed limited knowledge about sustainability in general, however interestingly, after sharing very brief examples of widely utilized examples of sustainability (e.g., Brundtland definition, going paperless, and solar panels), most expressed active interest in incremental information and knowledge regarding, sustainability, the SDGs, and their possible business interest in it.

\section{The SME/Community Finance Experience Is Trust "Heavy" but May Be Knowledge "Light". Coupled with Technological Change, This Can Be a (Future) Competitive Threat for Community Banks.}

SMEs gain knowledge via interactions with multiple actors: financers, suppliers, customers, family members, media, social clubs, associations, peers, etc. The SME leader's knowledge (at least potential) arguably increases over time. This is something a financial partner must continuously consider, and observably the ones regarded as being "good" financial partners and/or "good community bankers" prioritize both their own knowledge needs as well as the knowledge of importance to their SME clients. In this capacity, there was no significant evidence that smaller financiers outperform larger financiers, a finding in line with previous studies (Berger \& Udell, 2001; de la Torre et al., 2010; Hussain et al., 2021; Kang et al., 2013; Lehmann \& Neuberger, 2001; Lepoutre \& Heene, 2006; Santikian, 2014; Uchida et al., 2012). In general, this study found that most SMEs were not heavily reliant on their financiers for knowledge or 
meaning formations. Purpose, meaningfulness, and perceptions of communitylevel responsibility, for either the "community financiers" or SMEs themselves, also was not indicated as being directly tied to banking relationships, for the most part. This was a surprising development concerning business decisions as part of the SME/financier relationship, since it was hypothesized that SMEs actively sought guidance from their financiers and had more community-level expectations than was indicated by the sample population. Rather, navigational knowledge was attributed to third parties, mainly accountants, industry peers, and informal community leaders in a given landscape.

The research findings also suggested there might be some limitations in terms of perceived knowledge capacity on the part of financiers. This may be due to them holding back on subject matter to maintain a non-invasive presence in the SME's business matters. During discussion with bankers and SMEs, in terms of current modes of engagement, it was indicated that social and transactional dialogues occurred around mostly present or near-term needs. The social aspect of this existing model continues to offer some level of relationship "stickiness", but transaction-only relationships pose risks for both the SME and the financiers. For SMEs, this seems to be an underutilization and/or under-demanding (not a word) of a critical counterparty for knowledge resourcing. For financiers, the effect is one of potential service commoditization, particularly as technological advancements allow for more distant actors to compete with local or regional firms. Elyasiani and Goldberg (2004) identified the increasing importance of maintaining long-term relationship structures, embracement of technology, and 
non-standardized lending models that more intensely involve soft information exchanges as the persistent comparative advantages for community-oriented financiers (Elyasiani \& Goldberg, 2004). SMEs appear to solve for their knowledge needs outside of the relationship when required, and seemingly do not lose trust in their financiers if or when this occurs. In terms of transaction characteristics and bank competitiveness, a muted experiential response was also noted, namely that SMEs are not bothered by slightly higher rates or a narrower product offering to an extent where the interpersonal relationship suffers.

Community-level and individual knowledge formations directly contribute to meaningfulness, habits, values, and belief formations, which also were not reported as being actively influenced by financier relationships from the perspective of the SMEs. Likewise, the financiers who were interviewed also conveyed an intentional positioning that would not be construed as presumptive on the part of the SME, working to strike a balance between "friendly and functional servicing" and "letting the SME run their business", so only limited interactions on subject matter beyond what was either transactionally necessary or perceived as socially pleasing. So where does knowledge and meaning for SMEs come from? It seems that there are much stronger knowledge ties in SMEto-SME networks, which then in turn may influence community-level socialecological outcomes. As an example, one SME expressed "the town goes the way the X (influential SME leader in a rural community) goes" in response to questions about how both community financiers and other SMEs may prioritize 
SDG activeness and sustainability interests. Importantly, by "town" this description is referencing an inclusive landscape that includes other SMEs, political leaders, local financiers, and more. It also most likely comprises at least a partially shared, community-level values and belief system. Meaningfulness around the SDGs is founded, strengthened, minimized, or eliminated via interactional processes of information sharing, but, at present, this is not a key tenet of the SME financier interactive process. Yet, it could be, and furthermore it may offer a future, competitive advantage in relation to larger, more distant financiers.

This study does not dispute the existing literature themes for community finance's long-standing competitive advantages, namely relationship strength and community-centric modelling (soft information lending and locality-based charitable support in both rural and urban settings). However, as the landscape of financial interactions continues to be altered by new technologies, a persistent entrance of new participants (sometimes referred to in the literatures as de novo banks and also includes fintech, crowdfunding, etc.), and increasingly more network connection opportunities for SMEs, real threats may emerge. In the future, it may be even more challenging for community financiers to maintain their strength positioning that so heavily relies on the interpersonal relationship. In particular, it was observed that as SMEs were more mature/established this already becomes the case due to more complex product needs and extended financial service network opportunities; therefore, a higher need for knowledgeoriented, operational value creation were expressed (e.g., a specific type of 
financial product to meet long term financing requirement). On case specific bases, this is often due to the SME lifecycle - as a business grows, most likely the SME's knowledge accumulation needs increase, thus limiting the influence of the personal and social relationship between the SME and their financier. Regardless of SME size and place, meaning was mostly absent from SME perceptions of what they garnered from their bankers, regardless of their size or maturity, however it can be argued meaning conveyance is in fact occurring. Again, most SMEs banked with their community financiers for social reasons or transactional convenience and that was enough. But will this always be the case? Probably not, as exhibited by the firm's lifecycle growth example, and most likely no as the social-ecological landscape changes. As expressed by earlier scholarship, non-standardized lending models and commitments to technology will be critical for the future successes of community banks. Furthermore, the absence of directly associated meaning formations, even though habitually present in community finance processes, raises important questions about how financier strategies should evolve to deep value commitments via the relationships.

\section{SME Finance Complexities (Will) Persist........Community Banks Need to Keep Up}

The relationships between SMEs and community bankers are indeed dynamic and characteristically opaque, as described in earlier research. Financier size and geographic proximity seem to be less important than other factors. The opaqueness of SME finance will most likely persist for various 
reasons, namely their own continuous evolutions as they make decisions to survive, pivot, and grow. On one hand, no other actor profile in the modern economy enters and/or exits business participations as frequently as this population. On the other, consider the reach of SMEs in more advanced lifecycle phases (e.g., manufacturing business with 200 employees or a regional healthcare facility). They are deeply involved in global supply chains, provide lifecritical services, and often lead in technological innovation. Periodically, they fully transition into massive firms with expansive social-ecological influence (e.g., Amazon, Apple, and Tesla). SME navigations are complex throughout their lifecycles and their networked relationships can be expressed in numerous tones. There can be friendship, familial linkages, and often multi-nodal interfaces that blur the lines of professional and social networks. Such blended lines arguably contribute to the socio-cultural characteristics of landscapes of community interaction and the positioning of themselves and others on everything from politics to entertainment.

In any modern scenario, SME finance has numerous complexities that must be considered. Historically, much of the financial intermediation (banking) literature about SMEs has focused primarily on debt lending, access to capital, and the imperative of soft information as part of an SME's relationships with financiers of both choice and necessity. Santikian (2014) suggests such inquiries have been too narrowly concentrated on aspects of the SME finance process that are information oriented, excluding other activities that are central to how financiers engage with both their client and others in the industry. Berger and 
Udell (2006) also highlight the importance of understanding the vast and nuanced needs of SME financing needs, criticizing perspectives that are too homogenous in terms of technology delivery, service modeling, and lending policy. This study's findings align well with these points. There is also literature that points to long-standing misconceptions that smaller financiers always have stronger banking relationships and a greater desire to do business with SMEs than their much larger counterparts (Berger \& Udell, 2006; de la Torre et. al, 2010; Uchida et. al, 2012). This is not always the case. Also, in an earlier paper, Berger \& Udell (2002) also point to the lack of context regarding the organizational structuration of financial firms doing business with SMEs (Berger \& Udell, 2002). These articles are each relevant to the findings of this study as what SMEs seemingly value the most is understanding and effectively servicing their needs with the right types of financial service (including high quality information), regardless of who (or what size entity) does so.

Community finance and sustainable finance both invite much more research. As an example, more detailed risk-oriented questions on what happens in periods of crisis such as the COVID19 pandemic can offer important data to support business risk management studies. As an early indication related to community finance's role with time sensitive hazard management, this study offers at least some early insight. Community banks (either place based or specialized interest) seem to still be capable of outperforming their larger peers when immediate, time sensitive aid is required. The essence of this was largely found to be attributed to a combination effect of caring, comprehending, trust, 
open dialogues, and community banks being perceived as working to aggressively meet the needs of the SME. Nevertheless, it is important to understand indications of this level of service were not solely expressed as being present in only small, local banks. Similar positive experiences were indicated by SME interviewees who work closely with specialized and/or fintech tech providers, and in some cases when large bank relationships and service levels were perceived as strong. For the SMEs, size and locale seem to matter far less than interactive capacity and information sharing models (proactive preferred over reactive) that rise to their operational and strategic needs expectations - in both good times and bad.

It is important to clarify that the observance of light knowledge transference was not aligned with perceptions or feelings of distrust. Across the sample population, there was a general expression of relational trust, except for when there were perceived values breaches. These were expressed as interactions where the SME had felt wronged. In the literature, values are connected to meaning formations, and expressions of distrust formation that occurred as a result of feelings of wrongfulness offer another example of how community finance very capably and both intentionally and unintentionally shapes meaning (Aguinis \& Glavas, 2019; Bolis et al., 2014). Secondly, light knowledge does not infer or suggest a lack of intelligence. A few SMEs did share opinions that some financiers were "lucky", which is an interesting value judgement that may warrant incremental research, however this study's 
explorations of knowledge are apart from meanings associated with individual intelligence.

The socio-ecological literature on finance points to a need for better understanding the context of the relationships, the shared values (or not shared), and the role of trust in the interactional capacity of financiers and their clients. Arguably, for community financiers to innovate and become stronger knowledge players and facilitators of sustainable finance, understanding how they are dynamically involved in both attitudinal meaning and knowledge formations and salience for various subject matter is imperative.

Community Finance is a Community Field. This Contributes Largely to Its Role in (Potential) Interactive Capacity Development Across the SDG

\section{Landscape.}

Community finance, as a process that is co-experienced, is information rich, rich, rich! The dynamic relationships between SMEs and community bankers are comprised of interactions of varied importance, soft information, as well as sociocultural context was leveled up to give the process room for its potentially influence on par with other date profiles (e.g., hard info such as transactions, nodal interfaces, etc.). A diligent effort was made to analyze all forms of information as part of essential theme extraction. This included analyzing both active and passive (visible and invisible) information flows in the interview process, body language, choice observations (and notable constraints), and unconscious exposures with the latter being primarily represented by exposure to narratives that some sample participants were not aware of prior to 
their participation in the study. What was found was that soft information is the priority mechanism by which the SME and community banker co-operate, make decisions, and influence other fields of interaction and knowledge gains/losses at both the enterprise and community level. In particular, how SMEs work with financial partners that they (the SMEs) consider to be community banks and lenders was about what transpired between them socially or was perceived as morally and ethically in the positive support of businesses and the communit(ies) with which they identified. As a community field, community finance was central to what was perceived as realized/able across the landscape. As an example, what makes small town $\mathrm{X}$ a nice town? Or alternately, SME $\mathrm{X}$ was able to do certain things that were beneficial to the community's sociocultural and ecological well-being because of the facilitative mechanisms within the community finance field.

The implications for community finance's role in interactional capacity development are far-reaching when considered as a critical component for realizing the Sustainable Development Goals in both urban and rural settings. SDG information quality and delivery systems can and should be smartly curated to align with the interactional frames already in place within the community finance field that has been constructed and which is constantly re-constructed as SMEs and community bankers engage with each other and the broader community. 


\section{Yesterday's \& Tomorrow's 'Community Finance' - Considering the Micro and Macro for Resilience and Socio-ecologically Responsible Growth}

However, the study findings point to a disconnected presence for community finance and sustainable finance. This has both micro-level and macro-level implications. Community finance and sustainable finance were not actively or strategically linked, consciously or seemingly subconsciously, by the Midwest-based SME leaders and community bankers that participated in this study. Responding to this finding, is central to the applied purpose of my research agenda. Exploring a conceptual framework that places community finance at the center of sustainable finance may yield quite positive results for ground level attempts at SDG framework engagement. Centrally positioning community finance as the "grease" for interactional capacity development in relation to sustainable finance and the SDGs offers an already embedded vantage point from which to action community level and inclusive SDG agendas. This is beneficial on micro-levels for singular SMEs and community banks, respectively, as well as extends broadly to the community-level, industry-wide, regional, national, and global scale objectives. In particular, it aids with issues of both timeliness and scale. As an example, desired responses to the climate emergency/crisis may be conveyed more efficiently and effectively via an already embedded system of interactional exchange. Arguably, community banks have proven their merit for such positioning before, namely during the 2008 financial crisis most remained solvent while actively co-navigating varying levels of client default risk exposure and simultaneously responding to a stress tested regulatory 
environment. More recently, they are well documented has having been the nimble and enthusiastic partner for businesses working through their COVID19 pandemic driven situations, as was confirmed by the findings of this study. It seems that community banks are resilient as well as critical actors for the resilience of others.

Furthermore, community banks support entrepreneurial and other growthoriented strategies in different ways than larger institutions, largely because they may have more flexibility to rely on information that sits outside the transaction details. This is due to a variety of factors ranging from proximity and local ownership structures to operational management strategies that prioritize the needs of the community within which it operates. Thus, the community finance field is both a resilience oriented and entrepreneurial process. As community banks are already socially tied partners that have co-navigated good times and bad times for decades, key decision makers for nearly half the U.S. workforce, and are used to dynamic change events due to persistent entrepreneurial navigations, their potential impact as a facilitative leader of inclusive sustainable finance is massive. 


\section{Chapter 7 - Conclusion}

So, what does it all mean? Why is community finance in relation to sustainable finance, as well as continued reflections on its broader socialecological connections, an important topic? This is not a new area of scholarly interest as evidenced by the literature on the importance of networks and social relationships in finance (Carruthers \& Kim, 2011). As narratives of community finance and sustainable finance are highly observable across the sector, unpacking how individuals are experiencing community finance with, alongside, or separate from sustainable finance the linked SDG agenda can have widespread implications. Understanding SME and community banker relationships can offer insights into how communities as social structures are responding to $21^{\text {st }}$ century priorities of climate change, increased care of the earth's waters and lands, and one another. Community-level observations can be a complex unit of measurement, which is why focusing on the interactive capacity and transmission mechanisms that exists within the field of community finance is suggested.

After much reflection on the data findings, it appears community finance and sustainable finance are treated as different and perceived as unrelated in the current context of SME and community finance relationships. This concerns me, while simultaneously offering an invigorating challenge to make the case for embedding community finance within sustainable finance. So, what is the essence of community finance to SMEs? Per the findings, it is a contextual compilation of access, ease, approachability, localness, knowledge, caring, 
outreach, and responsiveness (in both normal and crisis periods). It is being there for and with the SME, often as part of a locality but not always.

Increasingly, it may be more about knowing and understanding the needs of the SME in the evolving navigations of entrepreneurship and SME leadership, all of which is offered via proactive and timely communications, robust servicing, risk ideation, product innovation, industry data, and more. In the context of the SDG objectives, when community finance as described above is placed within the sustainable finance agenda, powerful activeness can be unlocked at individual and community levels.

\section{Small Matters for a Sustainable Future}

Shared experience by SMEs and financiers in urban, rural, and even virtual settings offers beneficial contributions to the broader sociological and ecological discourses on the role of the finance industry in the globalized agenda of intended SDG alignments across diverse actor sets and institutions. Finance's structural embeddedness across societies mandates a continuous inquiry into how, why, and in what ways its presence contributes to the changing sustainability landscape in both highly developed and less developed nations. To date, SMEs and community finance entities have been understudied in this regard. Much of the scholarship on SME finance and relationships mostly focuses on active, purposeful information exchanges, as well as scaled actors. Better understanding how these other important actors engage with one another is imperative as such interactions directly affect dimensions of the human experience, narrative constructs, and the emergence of both habits and meaning 
formations concerning the essence of human-nature systemwide relationships. Prioritized economic development, finiteness in conservation approaches, and superior placements of technological innovation over the past several decades have grossly fallen short in delivering a sustainable future. Finance has been a critical actor in past scenarios and arguably will remain broadly influential in what is done in the years to come. As a result, the vast and active discourse on the role of finance in delivering the SDG targets is expanding, and arguably this study can contribute to the smart mobilization of communities of any shape or form to levels of responsiveness that will make a difference toward delivering the

SDG targets. This research contributes to this challenge by focusing on a couple key aspects of such engagements: 1) What is happening currently with SMEs and their financiers?, 2) What are they talking about, learning from one another?, and 3) How do they view community, sustainability, and the SDGs?

\section{Interactional Capacity and Context Matter}

It is important to acknowledge interactional landscapes involve both conscious and unconscious interactions that may be random, occasional, periodic or continuous. Involved actors are often part of a locality that has fuzzy boundaries and extra-local strong or weak ties, as described by Kaufman (1959), Wilkinson (1999), and Granovetter (1983). Furthermore, in an increasingly connected world, landscapes are now routinely exposed and affected by interactions that reach far beyond specific localities, thereby demonstrating persistent entry of new information, changing influences (power), and shifted orientations of interest, meaning, beliefs, and even habits of actor groups. Put 
simply, from a societal perspective, landscapes, and associated fields of interaction are greatly expanded as compared to just a few decades ago. Actors are active across landscapes in numerous ways that have direct effects on work, recreation, family life, faith activities, and both personal and group health and wellness. Non-human actors are also affected by such activeness, all of which seem to be capturable via understanding such interactions or the potential to relate to the SDGs, and in the context of the SME/financier relationships in communities (of interest or place). Why? This is largely due to 1 ) repeated interactions (which often include events of information exchange) convey landscape oriented meaning, both intentionally and unintentionally, 2) the social structure of community is minimally a co-constructor of shared realities in landscapes that overlap other landscapes, and 3) things constantly change. Of importance is the reality that these landscapes involve dynamic social processes within and across communities via multi-dimensional, interactional fields that are fluid, unbounded, and increasingly complex due to expanded connectedness that has been facilitated by technological advancements. In addition, in business as is the case with life, there can be extreme periods of stress, even crisis. The same is true from a planetary perspective, of which time we are now present concerning climate change, etc. It is the whole rationale for why the SDGs even exist. It is projected that individual and community level stressors will continue to increase. Factors of multi-dimensionality and persistently recreated structuration are exactly why the interaction itself matters, importantly, including not just the purposeful but also the accidental. As a result, degrees of possible influence by 
human actors, ranging from the individual person to the global institution, have substantially increased. The interactional landscape is bigger, involves more languages, diverse environments, and cultural histories. Due to the combination effects of such massive scale and dynamic elements of constant change, this research is important because it responds to the calls for future and continued research that focuses on contextual attributes of human systems of engagement. As offered by Qin et. al (2017), contextual analysis is not limited to specific or singular cases. Analytical approaches such as qualitative comparative analysis (QCA) can offer scaled syntheses for community contextual forces while simultaneously reducing single case bias and even single researcher bias (Qin et. al, 2017).

This study works to shed light on the micro context of community finance as experienced by SMEs in the midwestern US, yet its usefulness is broader, and intentioned to prompt continued discourse on the contextual attributes of community finance and the interactional capacity it presents for sustainable finance's systematized objectives. Financial interactions associated with community will most likely not cease. Thus, to work toward better understanding (and hopeful progress) of how perceivably small matters of micro level interactional capacity related to finance, and the qualitative factors that contribute to inclusive information exchange can contribute to broader societal well-being and sustainability. Why? Because small matters, can really matter. 


\section{Community Finance Can Be the Modern "Grease" for Sustainable Finance}

Unpacking the scale and effects (or effectiveness) of finance's role with the SDGs is a massive topic. Nevertheless, this research contributes to this important discourse by focusing on a population of interest that, to date, has not been largely engaged nor studied for its participatory role - past, current, or future - in sustainability pursuits. The scholarship on small-medium businesses and their role in the sustainable finance agenda, as part of achieving the SDGs, is quite limited thus far. Rather, the scholarship aligns SME finance with the community finance and relationship banking literatures. Nevertheless, this very alignment is where the "missed opportunities" can and should be grounded for its progressive inclusion in sustainable finance. SMEs and community bankers are well positioned to lead local and community level initiatives for SDG progress. Central to this study is the ideation of small-medium businesses and communityoriented (regardless of locale or size) financiers as the "grease" for facilitating on the ground knowledge exchange and action towards the SDGs. The emerging narrative of sustainable finance offers some indication of finance's next act objectives in this regard, but largely focuses on development agendas and large financier and multi-national corporation responses. Arguably, SMEs and smaller financiers are facing the same landscape(s) of social-ecological change due to network connectedness across the landscape (as an example, manufacturing oriented SMEs are often connected to global value chains). Given SMEs represents approximately $44 \%$ of the U.S. GDP, it seems appropriate to better understand their current orientations, potential contributions to, knowledge 
access limitations, perspectives, and beliefs around sustainability. Furthermore, the roles of both community finance and assumptions of community level interactive capacity are actively referenced in global sustainable development narratives and research scopes, yet much of the scholarship to date does not contextually explore or link the topics in applied research design. Global finance is held out as a special actor in some segments of the sustainable development, sustainability, and CSR/ESG literatures, given its facilitative dependency dynamic in relation to other industries, but no deep investigative inquiry was found that focused on the essence of community finance and its relation to sustainable finance. Recent work by Ziolo et. al (2019) reminds us that, "Conventional finance offers no room for the environment and society."(Ziolo et. al, 2019). Thus, better understanding both sustainable finance and community finance, and their trajectories, is critically important to explore how they may become as unconventional as necessary to support enhanced social-ecological landscapes. Arguably, community finance can offer both deeper insights and some creative solutions.

\section{Limitations}

This study offers important insights into what SMEs and their financiers are doing, today, in terms of community finance. It also offers a perspective from which to continue the investigation into what SMEs want, need, and expect from their financiers. In this aspect, it offers important and meaningful insights into community finance's current systematized interaction with priority relationships as well as early guideposts for change trends that will affect all parties. However, 
the study found that the population of interest's exposure to sustainable finance as part of the process was limited and requires more research. It is telling that sustainable finance and the SDGs were found nowhere in the Midwest, but that when mentioned in the course of interviews, the interest was perceived to be genuine. On this point, more work is required to expand the measurement of both absence and interest to better understand salience for the topic.

The COVID19 pandemic generated a time sensitive opportunity, and, as a result, the study was conducted over a specific period of time and in a limited geography. A broader study is suggested. In addition, SMEs work with financiers of varied shape, location, and organizational/legal structure. Local banks, cooperatives, credit unions, other lending entities promote their community finance commitment, so it is important to explore the community finance narrative and experience, as well as sustainable finance, in the context of these other financier profiles. As an example, it is unknown and out of scope for this current study if financial cooperatives or credit unions may be facilitating a more direct experiential connection between community finance and sustainable finance.

The study is a first level look at an exploratory framework for embedding community finance, as a process, within sustainable finance operationalizations. Continued work from this and other perspectives is suggested. Social-ecological change is constantly yielding both opportunities and challenges, and SMEs and financial sector actors are neither static nor unaffected by new concepts and meanings. It is also reasonable to acknowledge that the conceptualization of community as a social structure has its own limitations in theoretical design and 
attributes, but I maintain that it is a very useful concept for exploring how interactive capacity within communities can shape what matters. 


\section{References}

Aguinis, H., \& Glavas, A. (2019). On corporate social responsibility, sensemaking, and the search for meaningfulness through work. Journal of Management, 45(3), 1057-1086. https://doi.org/10.1177/0149206317691575

Archer, D. (2012). Finance as the key to unlocking community potential: Savings, finds and the ACCA programme. Environment and Urbanization, 24(2), 423440. https://doi.org/10.1177/0956247812449235

Avery, R.B. \& Samolyk, K.A. (2004). Bank consolidation and small business lending: The role of community banks. Journal of Financial Services Research, 25, 291-325. https://doi-

\section{org.proxy.mul.missouri.edu/10.1023/B:FINA.00000020667.29802.1C}

Axelrod, R. \& Bennett, S.D. (1993). A landscape theory of aggregation. British Journal of Political Science, 23(2), 211-233.

https://doi.org/10.1017/S000712340000973X

Bacon, M. (2012). Pragmatism. Polity Press.

Balfour, B., \& Alter, T. R. (2016). Mapping community innovation: Using social network analysis to map the interactional field, identify facilitators, and foster community development. Community Development, 47(4), 431-448. https://doi.org/10.1080/15575330.2016.1153493

Beck, T. (2013). Bank financing for SMEs - Lessons from the literature. National Institute Economic Review, 225(1). https://doi.org/10.1177/002795011322500105 
Berger, A. N., \& Udell, G. F. (2001). Small business credit availability and relationship lending: The importance of bank organisational structure. SSRN Electronic Journal. https://doi.org/10.2139/ssrn.285937

Berger, A. N., \& Udell, G. F. (2006). A more complete conceptual framework for SME finance. Journal of Banking and Finance, 30(11), 2945-2966. https://doi.org/10.1016/j.jbankfin.2006.05.008

Bernstein, R. J. (2010). The Pragmatic Turn. Polity Press.

Bevan, M. T. (2014). A method of phenomenological interviewing. Qualitative Health Research, 24(1), 136-144.

https://doi.org/10.1177/1049732313519710

Bogna, F., Raineri, A., \& Dell, G. (2020). Critical realism and constructivism: Merging research paradigms for a deeper qualitative study. Qualitative Research in Organizations and Management: An International Journal, 15(4), 461-484. https://doi.org/10.1108/QROM-06-2019-1778

Bolis, I., Morioka, S. N., \& Sznelwar, L. I. (2014). When sustainable development risks losing its meaning. Delimiting the concept with a comprehensive literature review and a conceptual model. Journal of Cleaner Production, 83, 7-20. https://doi.org/10.1016/j.jclepro.2014.06.041

Boot, A. W. A. (2000). Relationship banking: What do we know? Journal of Financial Intermediation, 9(1), 7-25. https://doi.org/10.1006/jin.2000.0282 Brammer, S., Millington, A., \& Rayton, B. (2007). The contribution of corporate social responsibility to organizational commitment. The International Journal of Human Resource Management, 18(10), 1701-1719. 
Bridger, J. C., \& Luloff, A. E. (1999). Toward an interactional approach to sustainable community development. Journal of Rural Studies, 15(4), 377387. https://doi.org/10.1016/S0743-0167(98)00076-X

Bridger, J. C., \& Luloff, A. E. (2001). Building the sustainable community: Is social capital the answer? Sociological Inquiry, 71(4), 458-472. https://doi.org/10.1111/j.1475-682X.2001.tb01127.x

Bright, A. D., \& Manfredo, M. J. (1997). The influence of balanced information on attitudes toward natural resource issues. Society and Natural Resources, 10(5), 469-483. https://doi.org/10.1080/08941929709381045

Butler, J., Bohensky, E., Suadnya, W., Yanuartati, Y., Handayani, T., Habibi, P., Puspadi, K., Skewes, T., Wise, R., Suharto, I., Park, S., \& Sutaryono, Y. (2016). Scenario planning to LEAP-frog the sustainable development goals: An adaptation pathways approach. Climate Risk Management, 12, 8399. https://doi.org/10.1016/i.crm.2015.11.003

Caelli, K. (2000). The changing face of phenomenological research: Traditional and American phenomenology in nursing. Qualitative Health Research, 10(3), 366-377. https://doi.org/10.1177/104973200129118507

Carruthers, B. G., \& Kim, J.-C. (2011). The sociology of finance. Annual Review of Sociology, 37. https://doi.org/10.1146/annurev-soc-081309-150129

Cheng, B., loannou, I., \& Serafeim, G. (2014). Corporate social responsibility and access to finance. Strategic Management Journal, 35(1), 1-23. https://doi.org/10.1002/smi.2131 
Coser, L. A. (2004). Masters of sociological thought: Ideas in historical and social context (2nd ed.). Wadsworth Group.

Costa-Climent, R., \& Martínez-Climent, C. (2018). Sustainable profitability of ethical and conventional banking. Contemporary Economics, 12(4 Special), 519-530. https://doi.org/10.5709/ce.1897-9254.294

Creswell, J. W. (2013). Qualitative Inquiry \& Research Design. SAGE Publications

Creswell, J. W. (2014). Research Design. SAGE Publications.

de la Torre, A., Martínez Pería, M. S., \& Schmukler, S. L. (2010). Bank involvement with SMEs: Beyond relationship lending. Journal of Banking and Finance, 34(9), 2280-2293. https://doi.org/10.1016/j.jbankfin.2010.02.014

Delanty, G. (2010). Community (2nd Edition). Routledge.

Desjarlais, R., \& Jason Throop, C. (2011). Phenomenological approaches in anthropology. Annual Review of Anthropology, 40. https://doi.org/10.1146/annurev-anthro-092010-153345

Deyoung, R., Hunter, W. C., \& Udell, G. F. (2004). The past, present, and probable future for community banks. Journal of Financial Services Research, 25(2-3), 85-133.

\section{https://doi.org/10.1023/b:fina.0000020656.65653.79}

Dickinson, R. E., \& Hawley, A. H. (1950). Human ecology--a theory of community structure. Economic Geography, 26(4), 328. https://doi.org/10.2307/141270 Disparities in PPP lending by race. (2021). The Institute. https://theinstitutenc.org/2021/03/disparities-in-ppp-lending-by-race/ 
Donker, H., Ng, A., \& Shao, P. (2020). Borrower distress and the efficiency of relationship banking. Journal of Banking and Finance, 112. https://doi.org/10.1016/j.jbankfin.2017.12.013

Dörry, S., \& Schulz, C. (2018). Green financing, interrupted. Potential directions for sustainable finance in Luxembourg. Local Environment, 23(7), 717-733. https://doi.org/10.1080/13549839.2018.1428792

Elyasiani, E., \& Goldberg, L. G. (2004). Relationship lending: A survey of the literature. Journal of Economics and Business, 56(4), 315-330. https://doi.org/10.1016/j.jeconbus.2004.03.003

Englander, M. (2012). The interview: Data collection in descriptive phenomenological human scientific research. Journal of Phenomenological Psychology, 43(1), 13-35. https://doi.org/10.1163/156916212X632943

Epstein, M. J., \& Roy, M.J. (2001). Sustainability in action: Identifying and measuring the key performance drivers. Long Range Planning, 34(5), 585604. https://doi.org/10.1016/S0024-6301(01)00084-X

Fairlie, R. W. (2020). The impact of Covid-19 on small business owners: The first three months after social-distancing restrictions (Working Paper 27462). National Bureau of Economic Research. http://www.nber.org/papers/w27462

Fatemi, A. M., \& Fooladi, I. J. (2013). Sustainable finance: A new paradigm. Global Finance Journal, 24(2), 101-

113. https://doi.org/10.1016/i.gfi.2013.07.006

Ferguson, N. (2008). The ascent of money: A financial history of the world. New York: Penguin Press. 
Finlay, L. (2014). Engaging Phenomenological Analysis. Qualitative Research in Psychology, 11(2), 121-141. https://doi.org/10.1080/14780887.2013.807899

Flaherty, J., \& Cope, M. R. (2016). A Reply to Theodori, Luloff, Brennan, and Bridger. Rural Sociology, 81(1), 46-65. https://doi.org/10.1111/ruso.12092

Forcadell, F. J., Aracil, E., \& Úbeda, F. (2019). The influence of innovation on corporate sustainability in the international banking industry. Sustainability (Switzerland), 11(11). https://doi.org/10.3390/su11113210

Fundera. (2021). https://fundera.org

Galaz, V., Crona, B., Dauriach, A., Scholtens, B., \& Steffen, W. (2018). Finance and the Earth system - Exploring the links between financial actors and nonlinear changes in the climate system. Global Environmental Change, 53, 296-302. https://doi.org/10.1016/i.gloenvcha.2018.09.008

Goglio, S., \& Catturani, I. (2019). Sustainable finance: A common ground for the future in Europe? In M. Migliorelli \& P. Dessertine (Eds). The rise of green finance in Europe. Palgrave Studies in Impact Finance. Palgrave Macmillan, Cham. https://doi.org/10.1007/978-3-030-22510-0 10

Granovetter, M., \& Swedberg, R. (2011). The sociology of economic life. Westview Press.

Greider, T., \& Garkovich, L. (2010). Landscapes: The social construction of nature and the environment. Rural Sociology, 59(1), 1-

24. https://doi.org/10.1111/j.1549-0831.1994.tb00519.x

Guerin, B. (1992). Behavior analysis and the social construction of knowledge. American psychologist, 47(11), 1423. 
Haack, S. (2006). Pragmatism, Old \& New. Prometheus Books.

Hale, G. (2012). Bank relationships, business cycles, and financial crises. Journal of International Economics, 88(2), 312-325. https://doi.org/10.1016/j.jinteco.2012.01.011

Hebb, T. (2013). Impact investing and responsible investing: What does it mean?, Journal of Sustainable Finance \& Investment, 3(2), 71-74, DOI: $10.1080 / 20430795.2013 .776255$

Hemingway, C. A. (2019). A big picture approach to (C)SR: Where are we now? Research in Ethical Issues in Organizations, 21. https://doi.org/10.1108/S1529-209620190000021005

Hendrickson, M. K., James, H. S., Kendall, A., \& Sanders, C. (2018). The assessment of fairness in agricultural markets. Geoforum, 96, 4150. https://doi.org/10.1016/j.geoforum.2018.07.020

Highfill, T., Cao, R., Schwinn, R., Prisinzano, R., \& Leung, D. (2020). Measuring the small business economy (WP2020-4). Bureau of Economic Analysis.

Hoffding, S., \& Martiny, K. (2016). Framing a phenomenological interview: What, why and how. Phenomenology and the Cognitive Sciences, 15(4), 539-564. https://doi.org/10.1007/s11097-015-9433-z

Humphreys, D., Singer, B., McGinley, K., Smith, R., Budds, J., Gabay, M., Bhagwat, S., de Jong, W., Newing, H., Cross, C., \& Satyal, P. (2019). SDG 17: Partnerships for the goals - Focus on forest finance and partnerships. In C. J. Pierce Colfer, G. Winkel, G. Galloway, P. Pacheco, P. Katila, \& W. de Jong (Eds.), Sustainable Development Goals: Their Impacts on Forests and 
People (pp. 541-576). Cambridge University Press. https://doi.org/DOI: undefined

Hussain, I., Durand, R. B., \& Harris, M. N. (2021). Relationship lending: A source of support or a means of exploitation? Global Finance Journal, 48. https://doi.org/10.1016/i.gfj.2020.100549Jackson, E.T. (2013) Interrogating the theory of change: Evaluating impact investing where it matters most, Journal of Sustainable Finance \& Investment, 3(2), 95-110, DOI: $10.1080 / 20430795.2013 .776257$

Jackson, E. T. (2013). Interrogating the theory of change: Evaluating impact investing where it matters most. Journal of Sustainable Finance \& Investment, 3(2), 95-110. https://doi.org/10.1080/20430795.2013.776257 Jensen, M. C., \& Meckling, W. H. (1976). Theory of the firm: Managerial behavior, agency costs and ownership structure. Journal of Financial Economics, 3(4), 305-360. https://doi.org/10.1016/0304-405X(76)90026-X Jensen, M. C., \& Meckling, W. H. (1994). The Nature of man. Journal of Applied Corporate Finance, Summer.

Jeucken, M. (2010). Sustainable Finance and Banking: The Financial Sector and the Future of the Planet. Earthscan. https://doi.org/10.4324/9781849776264

Johnson, M. H. (2012). Phenomenological approaches in landscape archaeology. Annual Review of Anthropology, 41. https://doi.org/10.1146/annurev-anthro-092611-145840 
Kang, E., Zardkoohi, A., Paetzold, R. L., \& Fraser, D. (2013). Relationship banking and escalating commitments to bad loans. Small Business Economics, 40(4), 899-910. https://doi.org/10.1007/s11187-011-9392-x

Kaufman, H. F. (1959). Toward an interactional conception of community. Social Forces, 38(1), 8-17.

Kautonen, T., Fredriksson, A., Minniti, M., \& Moro, A. (2020). Trust-based banking and SMEs' access to credit. Journal of Business Venturing Insights,

\section{4. https://doi.org/10.1016/i.jbvi.2020.e00191}

King, B. G., Felin, T., \& Whetten, D. A. (2010). Perspective-Finding the organization in organizational theory: A meta-theory of the organization as a social actor. Organization Science, 21(1), 290-

305. https://doi.org/10.1287/orsc. 1090.0443

Klassen, R. D., \& Vereecke, A. (2012). Social issues in supply chains:

Capabilities link responsibility, risk (opportunity), and performance. International Journal of production economics, 140(1), 103115.

Krannich, R. S., Luloff, A. E., \& Field, D. R. (2011). People, Places and Landscapes. In H. Decamps, B. Tress \& G. Tress (Eds.), Social Change in High Amenity Rural Areas, (Vol. 14), pp. 9-25.

Kuo, F. Y., \& Young, M. L. (2008). Predicting knowledge sharing practices through intention: A test of competing models. Computers in Human Behavior, 24(6), 2697-2722. 
La Torre, M. L., Trotta, A., Chiappini, H., \& Rizzello, A. (2019). Business models for sustainable finance: The case study of social impact bonds. Sustainability (Switzerland), 11(7). https://doi.org/10.3390/su11071887

Lagoarde-Segot, T. (2019). Sustainable finance. A critical realist perspective. Research in International Business and Finance, 47, 1-9. https://doi.org/10.1016/j.ribaf.2018.04.010

Landrum, N. E., \& Ohsowski, B. (2018). Identifying worldviews on corporate sustainability: A content analysis of corporate sustainability reports. Business Strategy and the Environment, 27(1), 128-151. https://doi.org/10.1002/bse.1989

Larson, E. C., Luloff, A. E., Bridger, J. C., \& Brennan, M. A. (2015). Community as a mechanism for transcending wellbeing at the individual, social, and ecological levels. Community Development, 46(4), 407-419. https://doi.org/10.1080/15575330.2015.1063074

Lauesen, L. M. (2016). The landscape and scale of social and sustainable finance. In O. Lehner (Ed.), Routledge Handbook of Social and Sustainable Finance. https://doi.org/10.4324/9781315772578

Lauterbach, A. A. (2018). Hermeneutic phenomenological interviewing: Going beyond semi-structured formats to help participants revisit experience. Qualitative Report, 23(11), 2883-2898.

Laverty, S. M. (2003). Hermeneutic phenomenology and phenomenology: A comparison of historical and methodological considerations. International 
Journal of Qualitative Methods, 2(3), 21-35.

https://doi.org/10.1177/160940690300200303

Lehmann, E., \& Neuberger, D. (2001). Do lending relationships matter?

Evidence from bank survey data in Germany. Journal of Economic Behavior and Organization, 45(4), 339-359. https://doi.org/10.1016/S01672681(01)00151-2

Lepoutre, J., \& Heene, A. (2006). Investigating the impact of firm size on small business social responsibility: A critical review. Journal of Business Ethics, 67(3), 257-273. https://doi.org/10.1007/s10551-006-9183-5

Levinson, S. (2006). Cognition at the heart of human interaction. Discourse Studies, 8(1), 85-93. https://doi.org/10.1177/1461445606059557

Li, Y., \& Foo, C. T. (2015). A sociological theory of corporate finance: Societal responsibility and cost of equity in China. Chinese Management Studies, 9(3), 269-294. https://doi.org/10.1108/CMS-12-2014-0232

Littig, B., \& Griessler, E. (2005). Social sustainability: A catchword between political pragmatism and social theory. International Journal of Sustainable Development, 8(1/2), 65. https://doi.org/10.1504/ijsd.2005.007375

Luloff, A. E. (2019). Community and social change: How do small communities act? In A. E. Luloff \& L. Swanson (Eds.), American Rural Communities. https://doi.org/10.4324/9780429042539-13

Mainzer, S., \& Luloff, A. E. (2017). Informing environmental problems through field analysis: Toward a community landscape theory of pro-environmental 
behavior. Community Development, 48(4), 483-498.

https://doi.org/10.1080/15575330.2017.1325918

Marquis, C., \& Lounsbury, M. (2007). Vive la résistance: Competing logics and the consolidation of U.S. community banking. Academy of Management Journal, 50(4), 799-820. https://doi.org/10.5465/AMJ.2007.26279172

Martínez, P., \& Rodríguez del Bosque, I. (2013). CSR and customer loyalty: The roles of trust, customer identification with the company and satisfaction. International Journal of Hospitality Management, 35, 89-99. https://doi.org/10.1016/j.ijhm.2013.05.009

McAdam, R., \& Keogh, W. (2004). Transitioning towards creativity and innovation measurement in SMEs. Creativity and Innovation Management, 13(2), 126-139. https://doi.org/https://doi.org/10.1111/j.09631690.2004.00300.x

McWilliams, A., Siegel, D. S., \& Wright, P. M. (2006). Corporate social responsibility: Strategic implications. Journal of Management Studies, 43(1), 1-18. https://doi.org/10.1111/j.1467-6486.2006.00580.x

Mendez, A., \& Houghton, D. P. (2020). Sustainable banking: The role of multilateral development banks as norm entrepreneurs. Sustainability (Switzerland), 12(3). https://doi.org/10.3390/su12030972

Merriam, S. B., \& Tisdell, E. J. (2015). Qualitative research: A guide to design and implementation (4th ed.). John Wiley \& Sons. 
Migliorelli, M. (2021). What do we mean by sustainable finance? Assessing existing frameworks and policy risks. Sustainability (Switzerland), 13(2), 117. https://doi.org/10.3390/su13020975

Misak, C. (2016). Cambridge Pragmatism. Oxford University Press.

Mizruchi, M. S., \& Fein, L. C. (1999). The social construction of organizational knowledge: A study of the uses of coercive, mimetic, and normative isomorphism. Administrative Science Quarterly, 44(4),

653. https://doi.org/10.2307/2667051

Moustakas, C. (1994). Phenomenological Research Methods. Sage Publications, Inc.

Mukherjee, A., \& Nath, P. (2003). A model of trust in online relationship banking. International Journal of Bank Marketing, 21(1), 5-15.

https://doi.org/10.1108/02652320310457767

Nájera-Sánchez, J. J. (2020). A systematic review of sustainable banking through a co-word analysis. Sustainability (Switzerland), 12(1). https://doi.org/10.3390/su12010278

Norton, L., \& Sliep, Y. (2018). A critical reflexive model: Working with life stories in health promotion education. South African Journal of Higher Education, 32(3). https://doi.org/10.20853/32-3-2523

Nosratabadi, S., Pinter, G., Mosavi, A., \& Semperger, S. (2020). Sustainable banking: Evaluation of the European business models. Sustainability (Switzerland), 12(6). https://doi.org/10.3390/su12062314 
O'Hara, J. K. (2017). Community banks and agricultural sales in rural states. The Review of Regional Studies, 47, 137-152.

Peoples, K. (2021). How to write a phenomenological dissertation. Sage Publications, Inc.

Pink, S. (2011). Multimodality, multisensoriality and ethnographic knowing: Social semiotics and the phenomenology of perception. Qualitative Research, 11(3), 261-276. https://doi.org/10.1177/1468794111399835

Qin, H., Sanders, C., Prasetyo, Y., Syukron, M., and Prentice, E. (2021). Exploring the dynamic relationships between risk perception and behavior in response to the Coronavirus Disease 2019. Social Science \& Medicine, (forthcoming).

Qin, H., Fan, Y., Tappmeyer, A., Freeman, K., Prentice, E., \& Gao, X. (2017). Capturing community context through qualitative comparative analysis of case studies. Human Ecology, 45(1), 103-109.

\section{https://doi.org/10.1007/s10745-016-9889-7}

Quatrini, S. (2021). Challenges and opportunities to scale up sustainable finance after the COVID-19 crisis: Lessons and promising innovations from science and practice. Ecosystem Services, 48. 101240. https://doi.org/10.1016/..ecoser.2020.101240

Raslavich, C. J., Breitenstein, E. C., Polson, C., DiChiara, R. M., Hanrahan, M., Cofer Jr., R. D., French, G., \& Hoople, D. (2020). FDIC Community Banking Study. Federal Deposit Insurance Corporation. 
Rauterkus, A., \& Munchus, G. (2014). Geographical location: Does distance matter or what is the value status of soft information? Journal of Small Business and Enterprise Development, 21(1), 87-99. https://doi.org/10.1108/JSBED-03-2013-0026

Rezende de Carvalho Ferreira, M., Amorim Sobreiro, V., Kimura, H., \& Luiz de Moraes Barboza, F. (2016). A systematic review of literature about finance and sustainability. Journal of Sustainable Finance and Investment, 6(2), 112-147. https://doi.org/10.1080/20430795.2016.1177438

Rizzello, A., \& Kabli, A. (2020). Sustainable financial partnerships for the SDGs: The case of social impact bonds. Sustainability (Switzerland), 12(13). https://doi.org/10.3390/su12135362SBA's Office of Advocacy. (2020). 2020 small business profile. https://advocacy.sba.gov

Rosen, M. A. (2018). Issues, concepts and applications for sustainability. Glocalism: Journal of Culture, Politics and Innovation, 3.

Salzmann, A.J. (2013). The integration of sustainability into the theory and practice of finance: an overview of the state of the art and outline of future developments. Journal of Business Economics, 83(6), 555-576. https://doi.org/10.1007/s11573-013-0667-3

Sanders, I. T. (1966). The Community: An introduction to a social system. The Ronald Press Company.

Santikian, L. (2014). The ties that bind: Bank relationships and small business lending. Journal of Financial Intermediation, 23(2), 177-213. https://doi.org/10.1016/j.jfi.2013.11.004 
Scholtens, B. (2006). Finance as a driver of corporate social responsibility. Journal of Business Ethics, 68(1), 19-33. https://doi.org/10.1007/s10551$\underline{006-90371}$

Schermer, H. \& Jary D. (2013). Interaction, form and the dialectical approachSimmel's analytical conceptual framework. In H. Schermer \& D. Jary (Eds.), Form and dialectic in Georg Simmel's sociology. Palgrave MacMillan, London. https://doi.org/10.1057/9781137276025 2

Score. (2021). https://score.org

Secinaro, S., Calandra, D., Petricean, D., \& Chmet, F. (2021). Social finance and banking research as a driver for sustainable development: A bibliometric analysis. Sustainability (Switzerland), 13(1), 1-18.

https://doi.org/10.3390/su13010330

Singer, A. E. (2009). Classical pragmatism and modern finance. Human Systems Management, 28(3), 83-92. https://doi.org/10.3233/HSM-20090700

Singer, B. (2016). Financing sustainable forest management in developing countries: the case for a holistic approach. International Forestry Review, 18(1), 96-109. https://doi.org/10.1505/146554816818206159

Smith, David Woodruff, "Phenomenology," The Stanford Encyclopedia of Philosophy (Summer 2018 edition), Edward N. Zalta (ed.), URL = $<$ https://plato.stanford.edu/archives/sum2018/entries/phenomenology/>.

Smulowitz, S.J., Rousseau, H.E. \& Bromiley, P. (2020). The behavioral theory of the (community-oriented) firm: The differing response of community- 
oriented firms to performance relative to aspirations. Strategic Management Journal, 41(6), 1023-1053. https://doi.org/10.1002/smj.3123

Srinivasan, A. (2014). Long run relationships in banking. Foundations and Trends in Finance, 8(2), 55-143. https://doi.org/10.1561/0500000041

Stambaugh, J., Lumpkin, G. T., Mitchell, R. K., Brigham, K., \& Cogliser, C. (2020). Competitive aggressiveness, community banking and performance. Journal of Strategy and Management, 13(2), 221-240.

https://doi.org/10.1108/JSMA-03-2019-0033

Stone, M. T., \& Nyaupane, G. (2014). Rethinking community in communitybased natural resource management. Community Development, 45(1), 1731. https://doi.org/10.1080/15575330.2013.844192

Theodori, G. L., \& Kyle, G. T. (2013). Community, place, and conservation. In W. P. et. al Stewart (Ed.), Place-Based Conservation: Perspectives from the Social Sciences (pp. 59-70). Publisher.

Theodori, G. L., Luloff, A. E., Brennan, M. A., \& Bridger, J. C. (2016). Making sense of "making sense": A critical response. Rural Sociology, 81(1), 35-45. https://doi.org/10.1111/ruso.12089

Tolbert, C. M., Mencken, F. C., Riggs, T. L., \& Li, J. (2014). Restructuring of the financial industry: The disappearance of locally owned traditional financial services in rural America. Rural Sociology, 79(3), 355-379. https://doi.org/10.1111/ruso.12037 
Turner, S., \& Endres, A. (2017). Strategies for enhancing small-business owners' success rates. International Journal of Applied Management and Technology, 16(1).

Uchida, H., Udell, G. F., \& Yamori, N. (2012). Loan officers and relationship lending to SMEs. Journal of Financial Intermediation, 21(1), 97-122. https://doi.org/10.1016/j.jfi.2011.06.002

Urban, M. A., \& Wójcik, D. (2019). Dirty banking: Probing the gap in sustainable finance. Sustainability (Switzerland), 11(6). https://doi.org/10.3390/su11061745

Utting, P., \& Zammit, A. (2008). United nations-business partnerships: Good intentions and contradictory agendas. Journal of Business Ethics, 90(S1), 39-56. https://doi.org/10.1007/s10551-008-9917-7

Vagle, M. D. (2018). Crafting phenomenological research, second edition. In Crafting Phenomenological Research (2 ${ }^{\text {nd }}$ Edition). Routledge. https://doi.org/10.4324/9781315173474

Valentine, K. D., Kopcha, T. J., \& Vagle, M. D. (2018). Phenomenological methodologies in the field of educational communications and technology. TechTrends, 62(5), 462-472. https://doi.org/10.1007/s11528-018-0317-2 Valentinov, V., \& Pies, I. (2018). From complexity reduction to moral character: A Deweyian reading of Luhmann's social systems theory. Systems Research and Behavioral Science, 35(6), 632-644.

van Manen, M. (2016). Phenomenology of Practice. Routledge. 
van Manen, M. (2017). But is it phenomenology? Qualitative Health Research, 27(6), 775-779. https://doi.org/10.1177/1049732317699570

Wilkinson, K. P. (1999). The community in rural America (2nd Edition). Social Ecology Press.

Willson, M.A. (2006). Technically together: Rethinking community within technosociety. Peter Lang Publishing, Inc.

Wilmoth, D. (2021). The effects of the COVID-19 pandemic on small businesses (Issue Bried 16). Office of Advocacy, U.S. Small Business Administration.

Wu, M.-W., \& Shen, C.-H. (2013). Corporate social responsibility in the banking industry: Motives and financial performance. Journal of Banking and Finance, 37(9), 3529-3547. https://doi.org/10.1016/j.jbankfin.2013.04.023 Yang, S., Ye, H., \& Zhu, Q. (2017). Do peer firms affect firm corporate social responsibility? Sustainability (Switzerland), 9(11). https://doi.org/10.3390/su9111967

Yip, A. W. H., \& Bocken, N. M. P. (2018). Sustainable business model archetypes for the banking industry. Journal of Cleaner Production, 174, 150-169. https://doi.org/10.1016/j.jclepro.2017.10.190

Zimmermann, S. (2019). Same but different: How and why banks approach sustainability. Sustainability (Switzerland), 11(8). https://doi.org/10.3390/su11082267

Ziolo, M., Filipiak, B. Z., Bąk, I., Cheba, K., Tîrca, D. M., \& Novo-Corti, I. (2019). Finance, sustainability and negative externalities. An overview of the 
European context. Sustainability, 11(15),

4249. https://doi.org/10.3390/su11154249

Zipin, L., S. \& Hattam, R. (2012). Countering and exceeding 'capital': A 'funds of knowledge' approach to the re-imagining community. Discourse: Studies in the Cultural Politics of Education, 33(2), 179-192. 


\section{VITA}

Christine Sanders is an experienced business leader and emergent researcher focused on the experiences of small-medium enterprises (SMEs) across urbanrural landscapes. Christine's research focuses on how SME leaders engage with "community finance" actors and navigate access to resources perceived as necessary for livelihood sustainability, risk management, resilience, and growth. Human-environment systems, community organization and interactional design, pragmatism, and the expanding "sustainable finance" narrative are central to her academic interests and professional pursuits. Christine has a Bachelor of Arts degree in Industrial Relations from Rockhurst University and a Master of Science degree in Agricultural Education \& Leadership from University of Missouri. She has recently fulfilled the requirements for a PhD from the University of Missouri's Division of Applied Social Sciences. She is actively engaged in projects focused on community finance, sustainable development, sustainability informatics, ruralurban networks, and economic sociology. 